\title{
Investigation of CRLH Transmission Lines for \\ Microwave Passive Components
}

by

Anas Alakhras, B.Sc.

A thesis submitted to the Faculty of Graduate and Postdoctoral Affairs in partial fulfillment of the requirements for the degree of

Master of Applied Science

in

Electrical and Computer Engineering

\author{
Ottawa-Carleton Institute for Electrical and Computer Engineering \\ Department of Electronics \\ Carleton University
}

Copyright $($ 2013, Anas Alakhras 
The undersigned recommend to the Faculty of Graduate and Postdoctoral Affairs acceptance of the thesis

\title{
Investigation of CRLH Transmission Lines for Microwave Passive Components
}

\author{
Submitted by \\ Anas Alakhras, B.Sc.
}

In partial fulfillment of the requirements for the degree of Master's of Applied Science

in Electrical and Computer Engineering

Professor Calvin Plett, Co-Supervisor

$\overline{\text { Adjunct Professor Ibrahim Haroun, Co-Supervisor }}$

Professor Calvin Plett

Chair, Department of Electronics

Ottawa-Carleton Institute for Electrical and Computer Engineering Carleton University

Department of Electronics

Ottawa, Ontario, Canada

2013 


\section{Abstract}

For decades, engineers have been searching for ways to reduce the size of electronic circuits while maintaining the efficiency and effectiveness of the circuits. At low frequencies of the microwave band, the size of the conventional transmission line becomes larger, because the wavelength is inversely proportional to the operating frequency; thus, it is needed to reduce the size to agree with the application requirements. Metamaterials are synthetic materials engineered to have a property that is not found in nature. They gain their properties not from their composition, but from their designed structures, and it could be used to reduce sizes of the electronics components.

The increasing demand for metamaterials technology to reduce sizes in electronic circuits, in conjunction with the advances in the Low Temperature Co-fired Ceramic (LTCC) technology, as well as the availability of $300 \mathrm{MHz}$ of bandwidth in the C-band (4.5-5.5 GHz), have created an interest in the development of a metamaterial transmission line which can be used to produce small-size millimeter-wave systems. In this research, the idea of using metamaterial depends on replacing each section of the conventional Right Handed (RH) transmission line, by equivalent Composite Right Left Handed Transmission Line (CRLHTL) implemented using lumped elements.

In this research, the CRLHTL was fabricated on ceramic substrate using 3-metal layers with dielectric constant $\left(\varepsilon_{\mathrm{r}}\right)$ of 7.1 and substrate thickness of $127 \mu \mathrm{m}$. The meas-

urement results were in agreement with the post-layout simulations over the bandwidth of 1-10 GHz. The on-wafer measurements of the transmission line were carried out using 
Agilent E8361A $10 \mathrm{MHz}$ to $67.0 \mathrm{GHz}$ vector network analyzer, and the EM simulation was performed by using an electromagnetic simulator (Agilent ADS).

As an example of an application, the CRLHTL was implemented in the design of a Branch Line Coupler (BLC). The design of the BLC used a CRLHTL to reduce the physical size of the quadrature wavelength elements of the coupler. The design exercise resulted in a coupler dimension of $5.924 \mathrm{~mm} \times 5.824 \mathrm{~mm}$ that is about $22.9 \%$ smaller compared to conventional BLC $(6.904 \mathrm{~mm} \times 6.778 \mathrm{~mm})$. Hence, the simulation results of the BLC achieved good performance over the bandwidth of 4.5-5.5 GHz compared to the conventional coupler. This type of coupler is widely used in microwave circuits, such as power combiners and dividers, balanced mixers, image rejection mixers, and balanced amplifiers. 


\section{Acknowledgement}

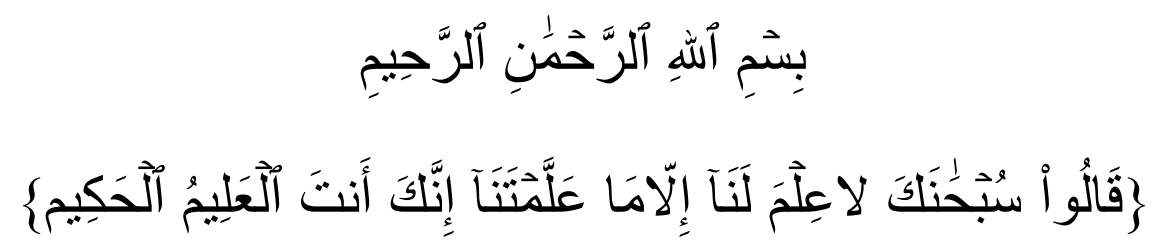

In the name of God, the most beneficent, the most merciful \{"They said: Be glorified! We have no knowledge saving that which Thou hast taught us. Lo! Thou, only Thou, art the Knower, the Wise"\}.

I thank God for blessing me with the knowledge I have attained and I pray that my work be of benefit for those who come after me.

In addition, I would like to thank my co-supervisor, Dr. Ibrahim Haroun, for his support, guidance, advices, and many suggestions throughout the course of my research.

I would like to express my thanks to Professor Calvin Plett, my supervisor, who is a continual source of inspiration and encouragement. In addition, I would like to thank the examining committee members of this thesis for their constructive comments.

Finally, I am very grateful to my family for extending their patience, support and love to me. Without them, this work would never have come into existence. 


\section{Table of Contents}

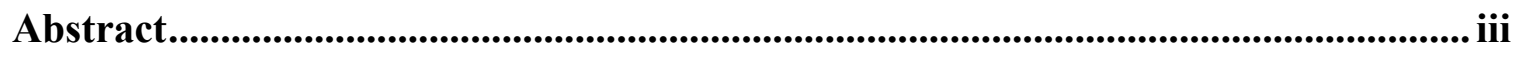

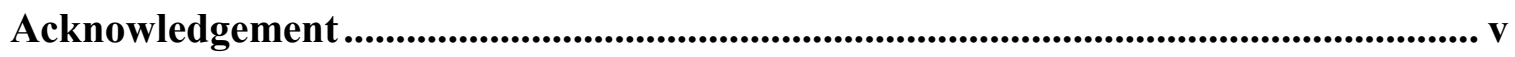

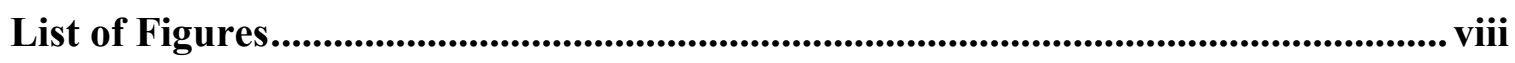

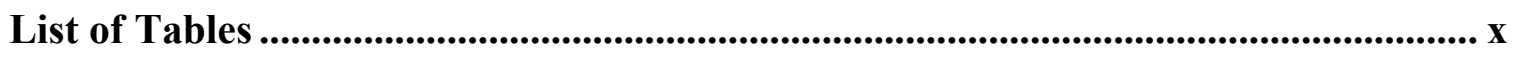

List of Symbols and Acronyms ........................................................................................ xi

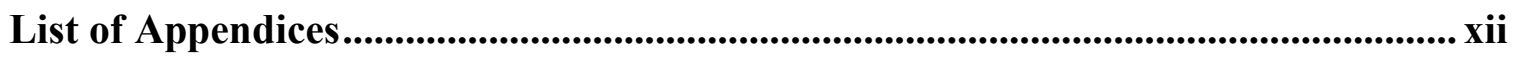

Chapter 1 Introduction ................................................................................................ 1

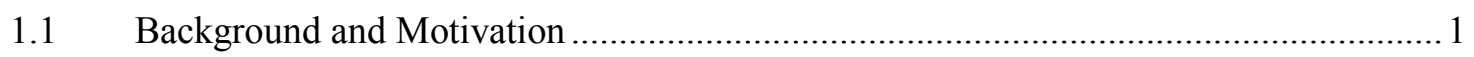

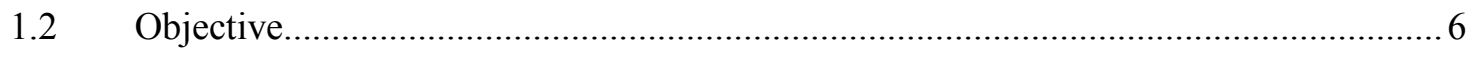

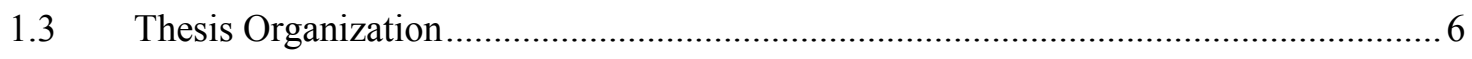

Chapter 2 Overview of Metamaterial Technology .................................................. 8

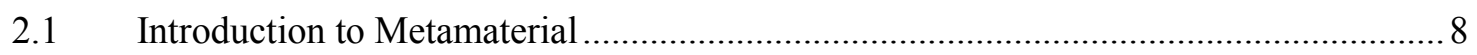

2.2 Composite Right Left-Handed (CRLH ) metamaterials .......................................... 12

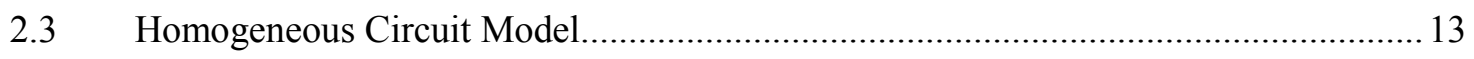

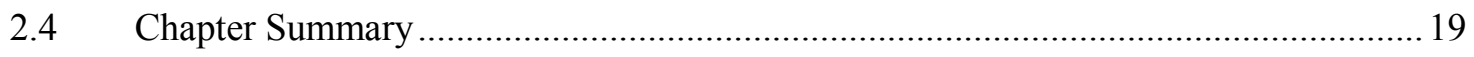

Chapter 3 Designof Metamaterial T.L using LTCC Technology...................... 20

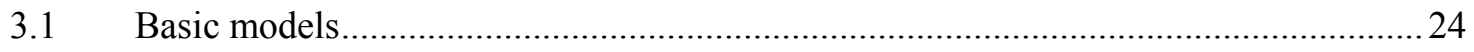

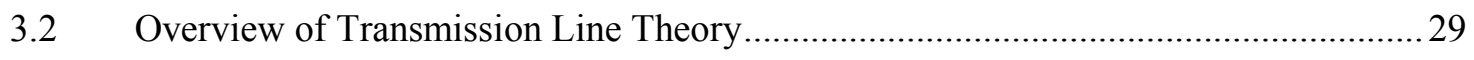

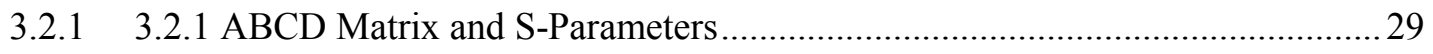

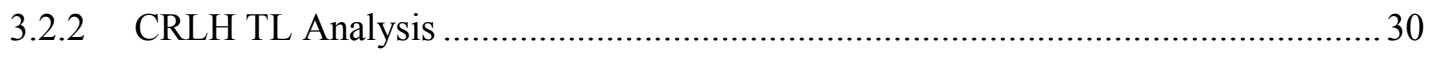

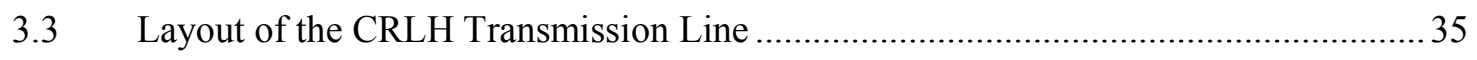

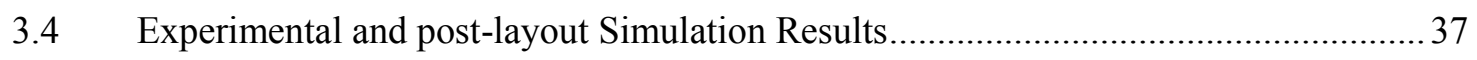

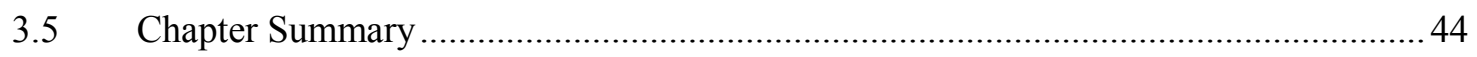

vi 
Chapter 4 Design of Branch Line Coupler Using CRLHTL............................... 46

4.1 Conventional Branch-Line Coupler in LTCC Technology ......................................... 47

4.2 Modeling and Simulation of $90^{\circ}$ conventional LTCC BLC .......................................48

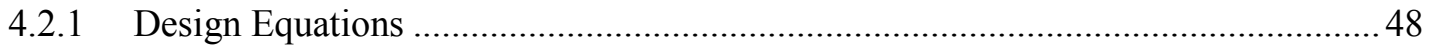

4.2.2 Simulation of Conventional BLC Using Lumped Elements ..................................49

4.3 Reducing the Branch Line Coupler's Size Using CRLHTL ......................................55

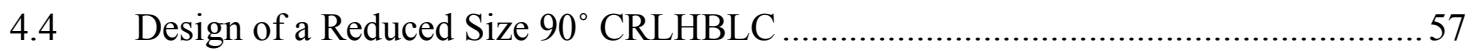

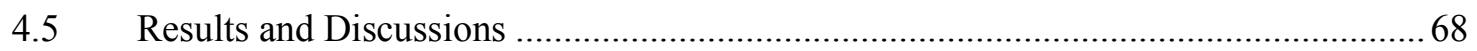

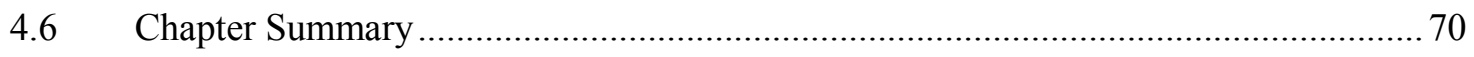

Chapter 5 Conclusions and Future Work.......................................................... 71

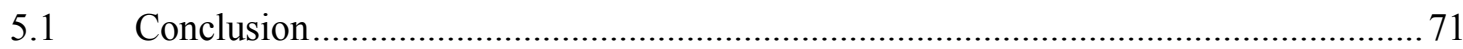

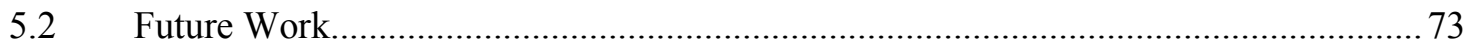

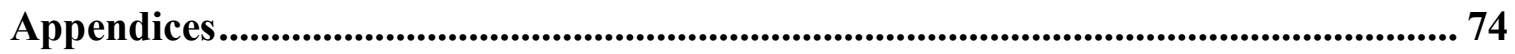

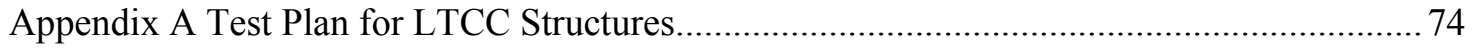

Appendix B Calculating the effective dielectric constant ..................................................... 77

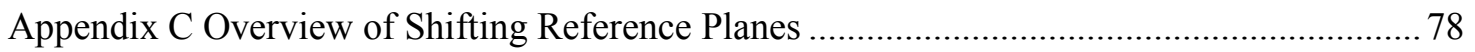

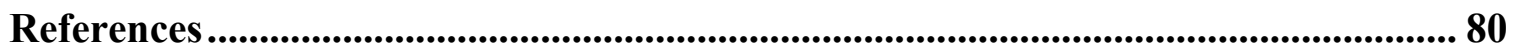




\section{List of Figures}

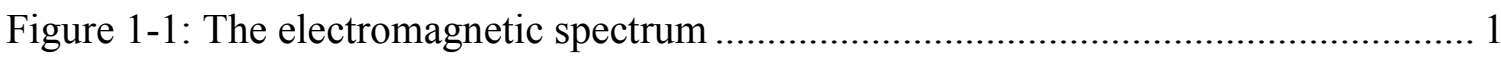

Figure 1-2: Chart represents the US usage of the frequency range ................................... 2

Figure 1-3: Typical wireless network ................................................................... 3

Figure 1-4: Block diagram of a balanced amplifier making use two BLCs ...................... 4

Figure 2-1: Typical index of refraction for the balanced and unbalanced CRLHTL ....... 10

Figure 2-2: Split ring resonator with an external magnetic field B ................................ 11

Figure 2-3: Equivalent circuit model for CRLH TL ..................................................... 13

Figure 2-4: Dispersion diagram of the CRLH TL for energy propagation....................... 16

Figure 2-5: Simplified equivalent circuit model and Dispersion diagram........................ 17

Figure 3-1: Lumped element model for CRLHTL, (b) Layout of the CRLHTL.............. 21

Figure 3-2: Physical structure for the LTCC with 6-Metal-Layers .................................. 24

Figure 3-3: Pure right-handed transmission line (PRHTL) ……………....................... 25

Figure 3-4: Response of the PRHTL and Dispersion diagram for the PRHTL ................. 25

Figure 3-5: Pure left-handed transmission line (PLHTL) ............................................. 26

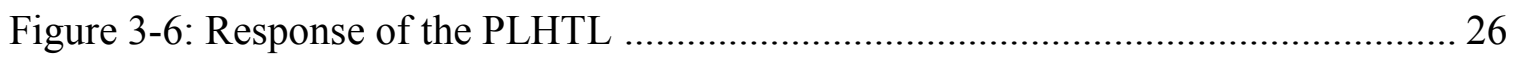

Figure 3-7: Equivalent circuit model of CRLHTL ......................................................... 27

Figure 3-8: The ABCD matrix Two port network …………………….................... 29

Figure 3-9: Equivalent circuit model of CRLHTL ........................................................ 30

Figure 3-10: Schematic diagram of the CRLHTL ……………………......................... 34

Figure 3-11: Simulated (a) Phase response, (b) S-parameters of the TL model............... 34

Figure 3-12: Dispersion diagram of the TL model ........................................................ 35

viii 


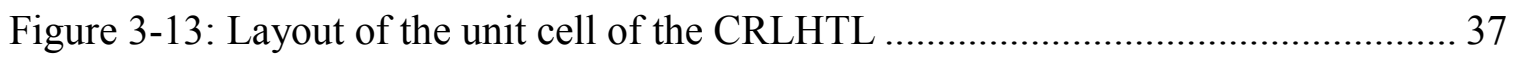

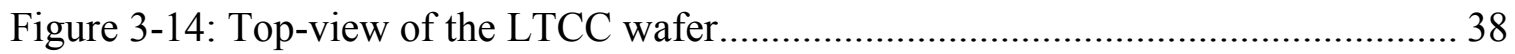

Figure 3-15: Measured and simulated of S(2,1) of the CRLHTL .................................. 40

Figure 3-16: Measured and simulated (a) Return loss, (b) Insertion loss ....................... 41

Figure 3-17: Dispersion diagram of the Measured CRLHTL ...................................... 43

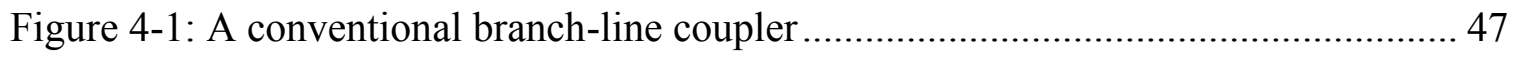

Figure 4-2: Conventional BLC uses lumped components ........................................ 49

Figure 4-3: Coupler model using microstrip elements ............................................ 51

Figure 4-4: Layout of the conventional BLC (a) 3-D view, (b) Dimensions view........... 53

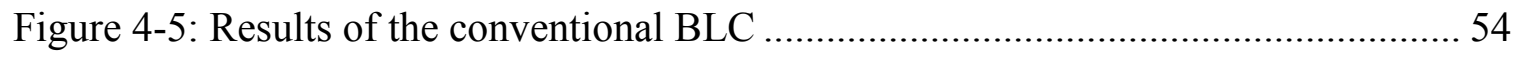

Figure 4-6: Schematic representation of (a) Conventional TL (b) CRLHTL ................... 55

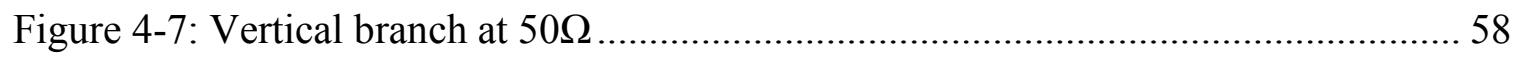

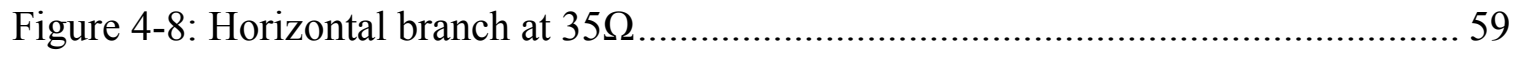

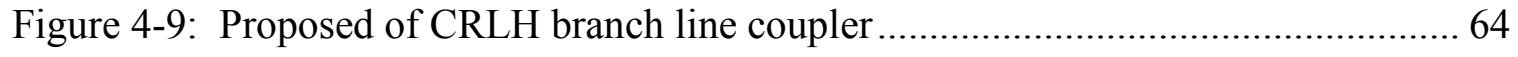

Figure 4-10: Configuration of the T-junction used in CRLHBLC design ....................... 64

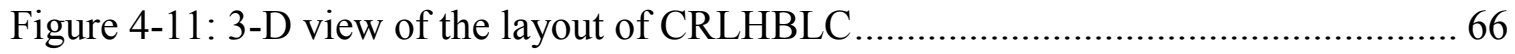

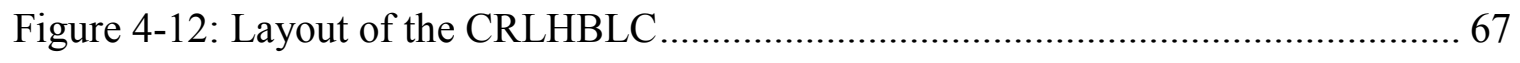

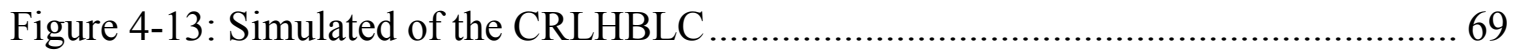




\section{List of Tables}

Table 3-1: Physical parameters of the ceramic substrate of LTCC tape system ............. 23

Table 3-2: Electrical parameters of the ceramic substrate of LTCC tape system............ 23

Table 4-1: Dimensions of the 35 ohms unit cell before and after adding the TL ............ 62

Table 4-2: Dimensions of the 50 ohms unit cell before and after adding the TL ............ 62

Table 5-5-1: Comparisons of the CRLHBLC with the conventional RHBLC ................ 72 


\section{List of Symbols and Acronyms}

\begin{tabular}{|l|l|l|}
\hline Name & Symbol & Unit \\
\hline Wavelength & $\lambda$ & Meter \\
\hline Frequency & $\mathrm{f}$ & Hertz \\
\hline Permeability & $\mu$ & Henry per meter \\
\hline Conductivity & $\sigma$ & Siemens per meter \\
\hline Resistivity & $\rho$ & Ohm per meter \\
\hline Time & $\mathrm{t}$ & Second \\
\hline Electric current & $\mathrm{I}$ & Ampere \\
\hline Permittivity & $\varepsilon$ & Farad per meter \\
\hline Inductance & $\mathrm{L}$ & Henry \\
\hline Capacitance & $\mathrm{C}$ & Farad \\
\hline Phase velocity & Vp & Meter per second \\
\hline Group velocity & Vg & Radians per second \\
\hline Per-unit-length impedance & $\mathrm{Z}^{\prime}$ & Ohm per meter \\
\hline Per-unit-length shunt admittance & $\mathrm{Y}^{\prime}$ & Siemens \\
\hline Times-unit-length capacitance & $\mathrm{C}_{\mathrm{L}}{ }^{\prime}$ & Farad per meter \\
\hline Per-unit-length capacitance & $\mathrm{C}_{\mathrm{R}}{ }^{\prime}$ & Farad per meter \\
\hline Times-unit-length inductance & $\mathrm{L}_{\mathrm{L}}{ }^{\prime}$ & Henry per meter \\
\hline Per-unit-length inductancee & $L_{\mathrm{R}}{ }^{\prime}$ & Henry per meter \\
\hline Speed of light in vacuum & $\mathrm{c}$ & Meter per second \\
\hline Angular Frequency & $\square$ & Radians per second \\
\hline Negative refractive index & NRI & \\
\hline Split Ring Resonators & SRRs & \\
\hline Transmission Line & TL & \\
\hline Composite Right Left Handed Transmission Line & CRLH TL \\
\hline Right Handed Transmission Line & RHTL & \\
\hline Metamaterial & MM & \\
\hline Purely Left Handed & PLH & \\
\hline Purely Right Handed & PRH & \\
\hline Complex propagation constant & $\gamma$ & \\
\hline Attenuation constant & $\alpha$ & \\
\hline Phase propagation constant & $\beta$ & \\
\hline Negative Refractive Index & NRI & \\
\hline & & \\
\hline
\end{tabular}




\section{List of Appendices}

Appendix A Test Plan for LTCC Structures.................................. 74

Appendix B Calculating the effective dielectric...............................76

Appendix C Overview of shifting Reference Plane...............................77 


\section{Chapter 1}

\section{Introduction}

\subsection{Background and Motivation}

Microwaves are electromagnetic waves operating in a frequency range between $300 \mathrm{MHz}$ and $300 \mathrm{GHz}$ [1]. Figure 1-1 shows the electromagnetic spectrum, which covers a wide range of wavelengths, where the microwaves wavelengths are between $1 \mathrm{~m}$ and $1 \mathrm{~mm}$.

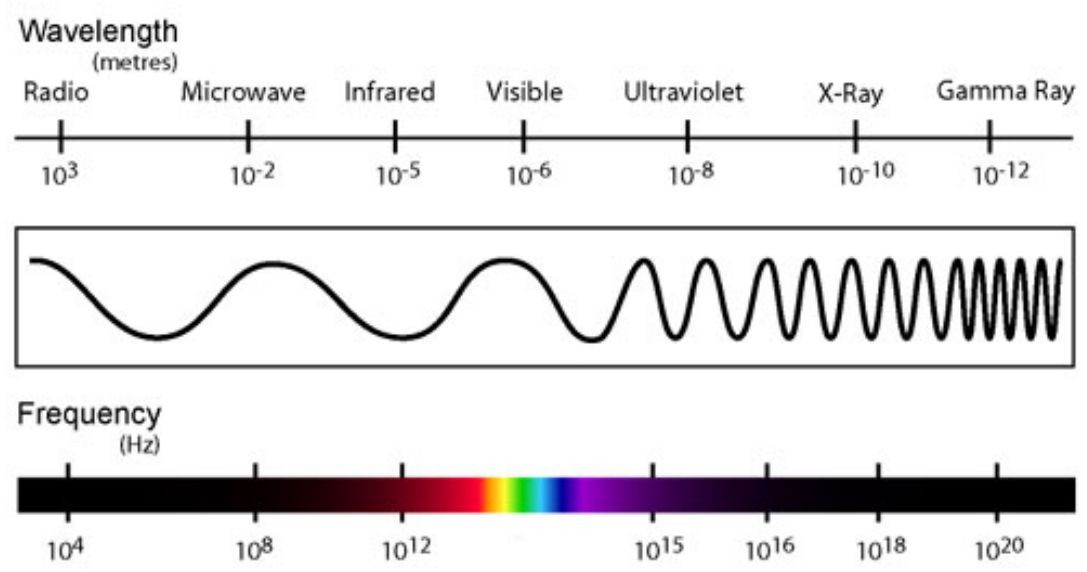

Figure 1-1: The electromagnetic spectrum [47]

The communications C-band was the first frequency band that was allocated for commercial telecommunications via satellites [1]. For instance, Figure 1-2 shows the 4 to $8 \mathrm{GHz}$ frequency range that is called the C-band, which can be used in radar systems and many other applications. 


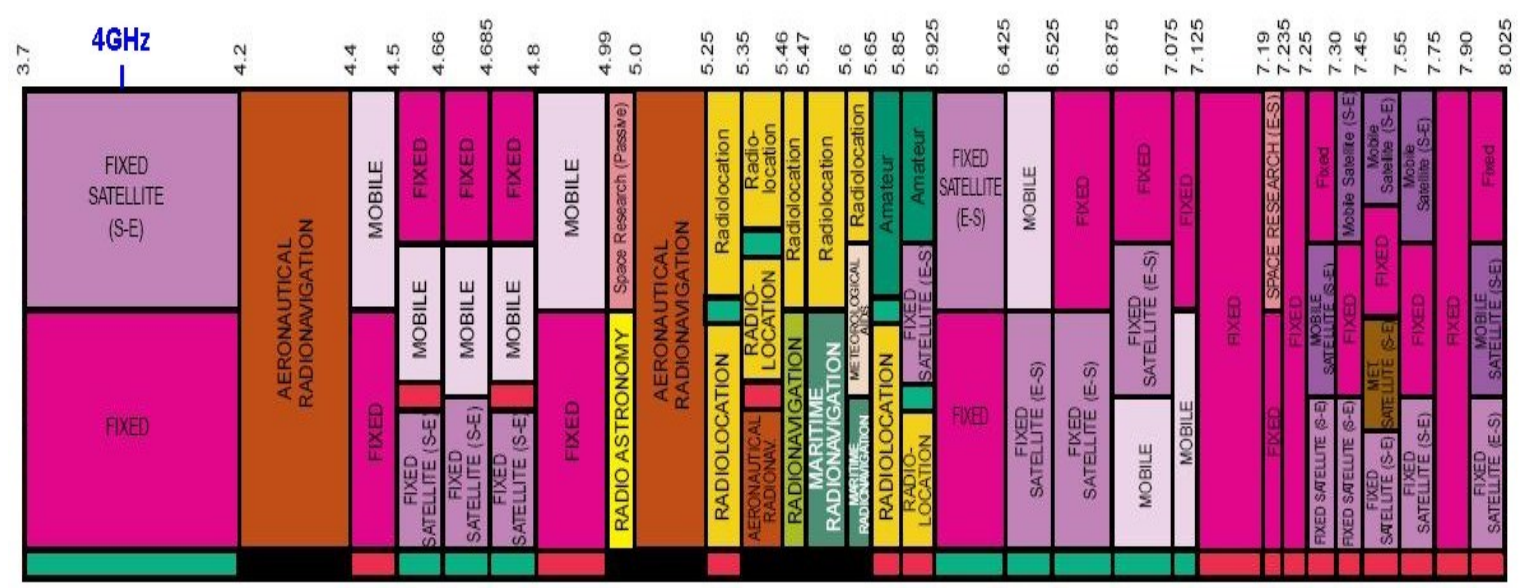

Figure 1-2: Chart represents the US usage of the frequency range [43].

Since the frequency is high and the wavelength approximates the physical dimension of the electrical devices, the distributed components in microwave designs became a typical way to design competitive and cost-effective microwave devices, instead of the conventional ways.

Metamaterials are used to develop the size-reduction and cost reduction of the microwave passive components, such as Branch Line Couplers (BLCs). There are many advantages of metamaterials over the conventional materials. For example, the structure of the metamaterial is an assembly of numerous individual elements shaped from conventional microscopic materials, such as metals or plastics. Since the geometry shape, size, direction and arrangement can affect the EM waves in an unconventional manner, it is possible to create material properties, which are unachievable with conventional materials. In addition, metamaterials achieve desired effects by incorporating structural elements of subwavelength sizes, leads to features that are smaller than the wavelength of the waves they 
affect [53]. There are many advantages of using the LTCC (low temperature co-fired ceramic) technology process, such as [2]

Low processing temperature and high conductivity metals (gold and alumina).

Good dielectric thickness control, and high number of conductive layers, up to 12. High integration density such as

Conductor line width and spacing down to $50 \mu \mathrm{m}$.

Buried via structures.

Low cost technology and high flexibility.

All the previous reasons have created an interest in developing a metamaterial transmission line to implement passive devices at $\mathrm{C}$ band frequencies, such as a branch line coupler, passive devices used in front-end circuits for many microwave applications [2][3]. These couplers play a major role in producing balanced amplifiers, which in turn form the basis of many microwaves applications in telecommunication systems. Figure 1-3 shows a typical wireless network, these networks are assembled of several integrated components, such as a balanced amplifier.

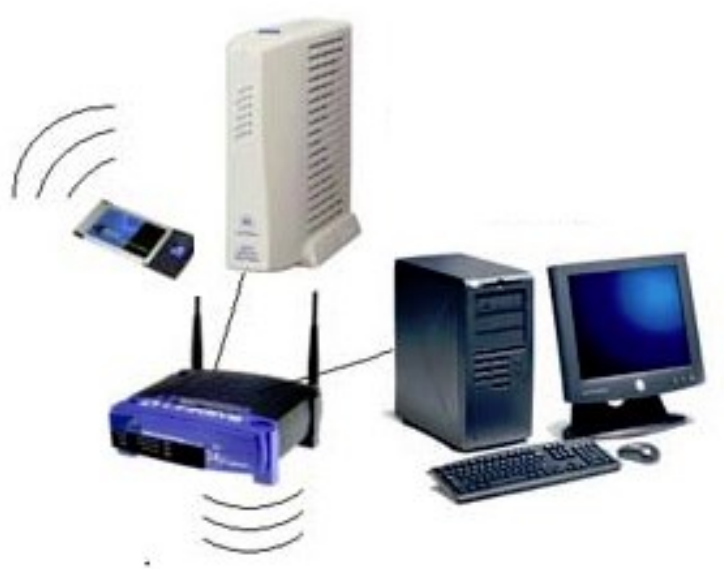

Figure 1-3: Typical wireless network [48] 
Figure 1-4 shows a balanced amplifier block diagram, utilizing two amplifiers in parallel, and making use of two branch line couplers at the input and output stage, respectively. The balanced amplifier can be designed using different types of couplers, such as Branch Line coupler (BLC) as an input and output port. And the balanced amplifier can be used for wireless infrastructure receiver front-end applications.

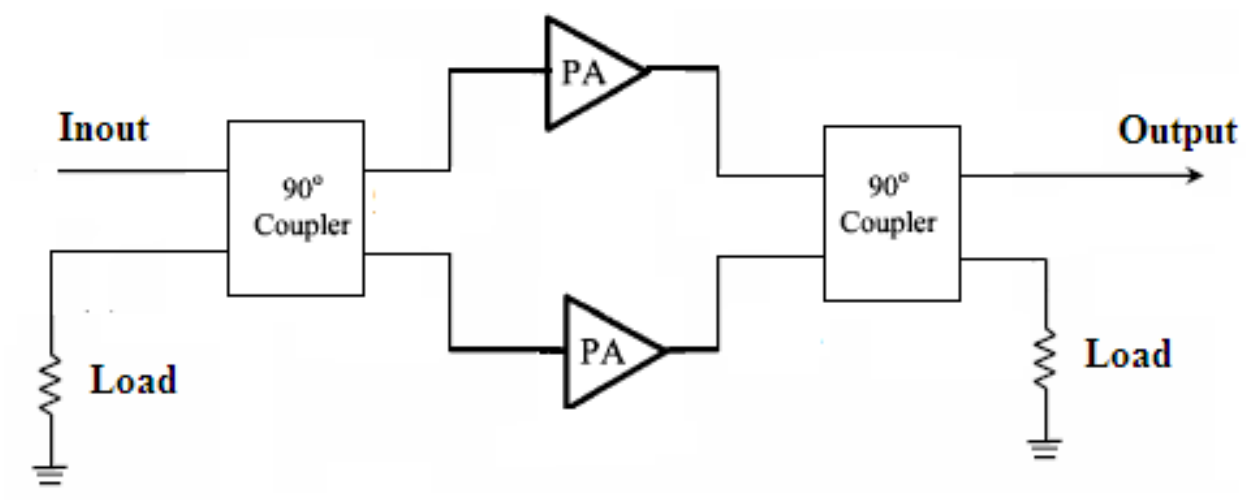

Figure 1-4: Block diagram of a balanced amplifier making use two BLCs

Several different approaches are proposed to realize the metamaterial transmission lines. In [55], C. Liu and K. Huang in chapter 13 focused on the concept of metamaterial transmission line and the circuit models, which facilitate the analysis of metamaterial transmission lines. As well as the relations between composite right/left-handed transmission line and band-pass filters they discussed some applications of metamaterial transmission lines in microwave components, such as power dividers.

In [5], Bo Zhao designed a new type of the microstrip resonant antenna using interdigital capacitor and shorted stub inductor, to form the physical size of the Composite Right Left-Handed (CRLH) transmission line. 
In [38], Hui-yong and Wang proposed a novel compact-size branch-line coupler using composite right left-handed transmission lines. This novel coupler occupies only $22.8 \%$ of the area of the conventional approach at $0.7 \mathrm{GHz}$. The overall size of the BLC and conventional branch-line coupler operating at $0.7 \mathrm{GHz}$ is $36 \times 35.2 \mathrm{~mm}^{2}$ and $72.37 \mathrm{x} 76.82$ $\mathrm{mm}^{2}$, respectively.

In [41], G. Elefthariades and R. Islam presented the Negative-Refractive-Index Transmission-Line (NRI-TL) constructed with series capacitors and shunt inductors. These NRI-TL media exhibit properties that can be utilized for miniaturizing microwave devices and antennas. They proposed a compact size of an antenna, a power divider, and a branch-line coupler using NRI-TL compared to its conventional microstrip at $2.05 \mathrm{GHz}$, it exhibits $72 \%$ by area size reduction and similar performance at a slightly reduced bandwidth.

In [39], J. Liang and G. Wang presented a novel resonate-type composite right left handed transmission line (CRLH TL) based on a high-low impedance section, a capacitive gap on the conductor strip and split ring resonators. For application, a compact branch- line coupler (BLC) centered at $0.88 \mathrm{GHz}$ (GSM band) was designed, fabricated and measured. The measurement results indicated that the coupler achieves $81 \%$ size reduction and a good in-band performance. The BLC is fabricated on the substrate RT/duroid 5880 with constant dielectric of 2.2 , thickness $\mathrm{h}=0.508 \mathrm{~mm}$.

In [40], Hsin. Lu and Yen. Kuo presented the MIM capacitor and shunt spiral inductor where they realized that the CRLH transmission lines in LTCC for a dual band branchline coupler with only series MIM capacitor and shunt spiral inductor in layout. Meas- 
urement results show dual-band coupling responses at $2.4 \mathrm{GHz}$ and $5.2 \mathrm{GHz}$ and a good performance has been achieved.

\subsection{Objective}

The thesis objectives are as follows:

To review the metamaterial transmission line design techniques based on a multilayer ceramic LTCC technology process.

To design, build and test a Composite Right Left Handed Transmission Line (CRLHTL) using ceramic substrate LTCC technology at C-band frequency.

To compare designs in terms of performance and compact size for the CRLH branch line coupler and conventional coupler using the LTCC process at C-band frequency.

\subsection{Thesis Organization}

There are five parts in this thesis. The first part is the introductory chapter outlining the thesis motivation, scope of work and objectives.

The second part is the theory chapter. It contains a background regarding metamaterial technology. It also contains a comprehensive review of wave propagation in transmission lines using Maxwell's equations, and an equivalent circuit model for the CRLHTL. The third part is the application chapter. The main focus is the design of a transmission line using CRLH metamaterial. In addition, the chapter describes the analysis and characterization of the composite right left handed transmission line, and introduces a short overview of phase shift reference plane method, as well as the relation between the 6 
ABCD matrix and S-parameters. The circuit model of the CRLHTL and layout model are also presented and discussed; the chapter also focuses on the design approach of a CRLHTL using LTCC process and the experimental characterization using electromagnetic simulation and network analyzer, respectively. The comparison between the measurement and EM simulation are furthermore presented and discussed.

The fourth part is the case study chapter. It contains the design of a C-band LTCC Branch Line Coupler (BLC). In addition, it contains the outcome of the simulation taken from both the ideal and CRLH branch line couplers. Comparisons between the simulation results are also discussed.

The fifth part is the conclusion chapter. It summarizes the work presented in this thesis and provides suggestions and recommendations for future work in this area of study. 


\section{Chapter 2}

\section{Overview of Metamaterial Technology}

\subsection{Introduction to Metamaterial}

In the real world, the permittivity and permeability for the majority of materials are positive. Metamaterial refers to the artificially constructed material, which is characterized by some properties that cannot be found in the surrounding environment, and have periodic dielectric structures that exhibit properties of Negative Refraction Index (NRI) and negative phase velocity. This type of material was first proposed by Veselago, who named these materials Left Handed Materials (LHMs) because of their unique properties of negative permittivity $(\boldsymbol{\varepsilon})$ and negative permeability $(\mu)$ which lead to create a Negative Refractive Index (NRI) [3][4].

Metamaterial became an exciting topic for researchers because of its capability of producing a negative refractive index that helped in building the homogeneous circuit models. The left-handed metamaterials have the negative permittivity and permeability simultaneously. This provides the left-handed metamaterials various new properties in comparison with the traditional right-handed $(\mathrm{RH})$ material [3]. The electromagnetic metamaterials are synthetically structured composite materials that can be engineered to have chosen electromagnetic properties that allow researchers to provide new applications [6][7]. The understanding of metamaterials comes from the understanding of the material response to electromagnetic waves in homogeneous materials, which is controlled by two parameters, permittivity $\varepsilon(\omega)$ and permeability $\mu(\omega)$. The permittivity describes the response of material to the electric component of electromagnetic waves and light, and the 8 
permeability describes the magnetic component at a frequency $\omega$. Both the permeability and permittivity are considered frequency dependent with complex quantities that describe the response of the material to electromagnetic radiation at a given frequency [45]. A commonly used electromagnetic parameter is that of the index of refraction, which is defined as

$\mathrm{n}^{2}(\omega)=\varepsilon(\omega) \mu(\omega)$

where $\mathrm{n}$ is the refractive index.

The index of refraction measures the speed of an electromagnetic wave as it propagates within a material, and measures the deflection of a beam of light as it crosses the interface between two materials having different values for their refractive indices [45]. Early in the seventeenth century, Willebrord Snell provided a quantitative measure of this bending as in [42] [43], and shows that,

$\mathrm{n}_{1} \sin \theta_{1}=\mathrm{n}_{2} \sin \theta_{2}$

where $\theta_{1}$ and $\theta_{2}$ are the angles of incidence of a ray crossing the interface between two media with refractive indices $n_{1}$ and $n_{2}$.

On the other hand, from $n^{2}=\varepsilon_{r} \mu_{r}$, it is not evident that negative permeability and permittivity lead to a negative reflection index. The negative solution for the index of refraction is selected in left-handed-materials to satisfy the condition that in refracted beams, the power flows away from the interface on the grounds of causality [3], [8]. Figure 2-1 illustrate the index of refraction diagram, where the refractive index is negative when $\omega<\omega_{0}$, and positive when $\omega>\omega_{0}$, where $\omega_{0}$ is the center frequency [3]-[15]. 


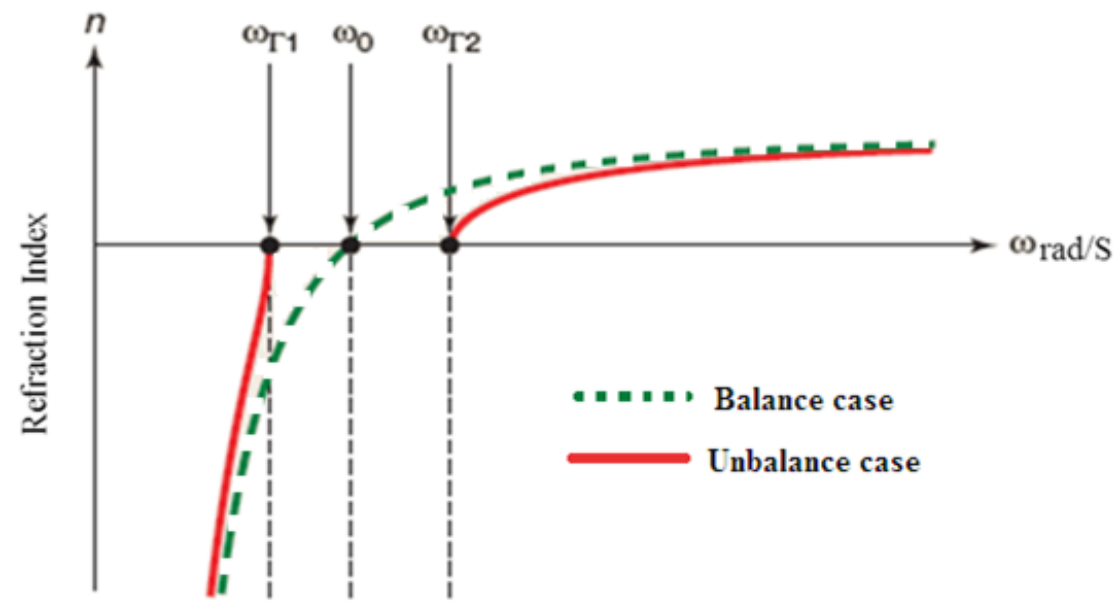

Figure 2-1: Typical index of refraction for the balanced and unbalanced CRLHTL [3]

The Russian physicist Victor Veselago [44] described the negative index of refraction. He realized that if a material was found that had negative values electric $\varepsilon(\omega)<0$ and magnetic $\mu(\omega)<0$ response functions, then its index of refraction would also be negative, $\mathrm{n}(\omega)<0$, and the artificial structure of metamaterials have the capability to control the magnetic and the electric responses over a broad frequency range.

In 1999, Pendry proposed the split ring resonator, which considered the typical element used for response to the magnetic component of the electromagnetic field [45]. Split ring resonators (SRR) can be made up of two microstrip rings with a slit in between. The desired negative index metamaterial properties at some frequencies can be controlled by several parameters such as the width and height of the microstrips, distance between the rings, size of the slit, material properties of the rings, substrate, and surrounding medium. The mechanism of the SRR can be thought of as an LC resonator. A time changing magnetic field polarized perpendicular to the plane of the split ring resonator will induce circulating currents according to Faraday's law. Because of the slit gap in the split ring res10 
onator, this circulating current will result in a buildup of charge across the slit gap with the energy stored in its equivalent capacitance. Figure 2-2 shows a split ring resonator with an external magnetic field B incident upon it. The split ring resonator can be considered as a simple LC circuit, with a resonance frequency of $\omega_{0}=\sqrt{\frac{1}{L C}}$, whereas the inductance results from the current path of the split ring resonator. For frequencies below $\omega_{0}$, currents in the SRR can keep up with the driving force produced by the externally changing magnetic field and a positive response is achieved. However, increasing the frequency will increase the magnetic B-field, which causes the currents to eventually lag, resulting in an out-of-phase or negative response [43] [45].

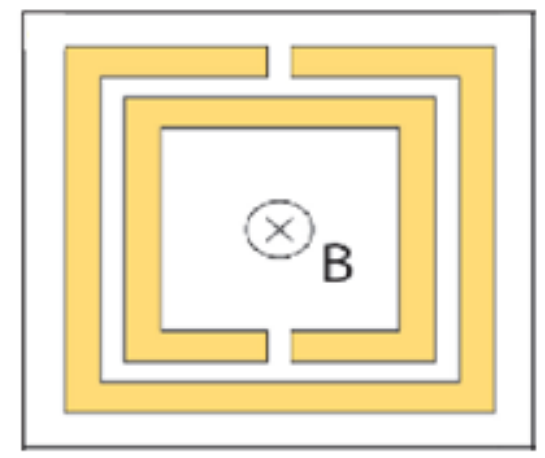

Figure 2-2: Split ring resonator with an external magnetic field B [45]

The elements of the branch line coupler of this work are implemented using composite right left handed transmission lines. The grounded stub inductor and the MIM-capacitor are combined for the CRLH phenomenon. The design of the branch line couplers is based on the idea of controlling the impedance of the longitudinal and the transverse branches by changing the values of the MIM-capacitor and stub inductor to reduce the size. The 
unit cell size of the transmission line $(p)$ is much smaller than the guided wavelength $(\lambda \mathrm{g})$, i.e. $(p<\lambda \mathrm{g})$, the physical length can be obtained as follows [3]

$p=\frac{\pi}{2 . \beta}=\frac{\pi . \mathrm{c}}{2 . \omega}=\frac{\mathrm{c}}{4 . \mathrm{f}}=\frac{\lambda}{4}$

$\lambda_{\mathrm{g}}=\frac{\lambda}{\sqrt{\varepsilon_{\mathrm{eff}}}}$

$p=0.25 \lambda_{\mathrm{g}} \times \sqrt{\varepsilon_{\mathrm{eff}}}$

where $\lambda$ is the wavelength in free space, $\varepsilon_{e f f}$ is the effective dielectric constant, where more details are listed in (appendix B).

The metamaterila transmission line characterized by effective homogeneous permittivity and permeability parameters with negative values that leads to a negative refractive index transmission line [52], and supports backward wave propagation with phase increasing away from the source [8].

\subsection{Composite Right Left-Handed (CRLH ) metamaterials}

Composite Right Left-Handed (CRLH) metamaterials are metamaterial with both righthanded and left-handed properties. It has demonstrated by several applications in microwave systems, such materials have a capability to reduce the number of components in wireless communication systems. The properties of the CRLH metamaterial can be analyzed using the transmission line theory [3][10]. 


\subsection{Homogeneous Circuit Model}

The term Left-Handed Material (LHM) is generally used in this thesis. Other terms that can be used are,

Negative Refractive Index (NRI) material, and

Backward-wave (BW) media.

Materials with only one negative parameter between permittivity and permeability are called single negative (SNG). This terminology aspect of metamaterial can be found in $[3,10-12]$.

The matching homogeneous transmission line modules for the purely Right-Handed (PRH), purely Left-Handed (PLH), and the CRLH transmission lines which are shown in

Figure 2-3 [3].

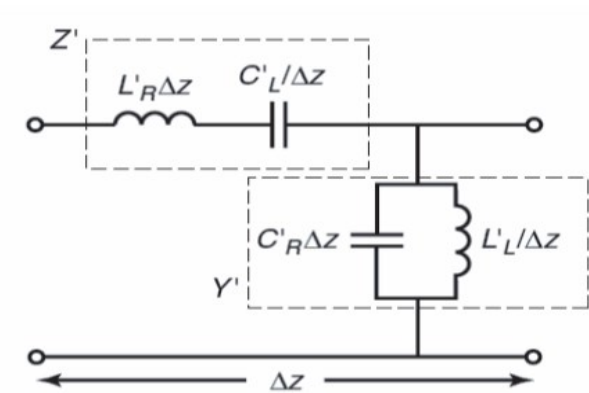

(a)

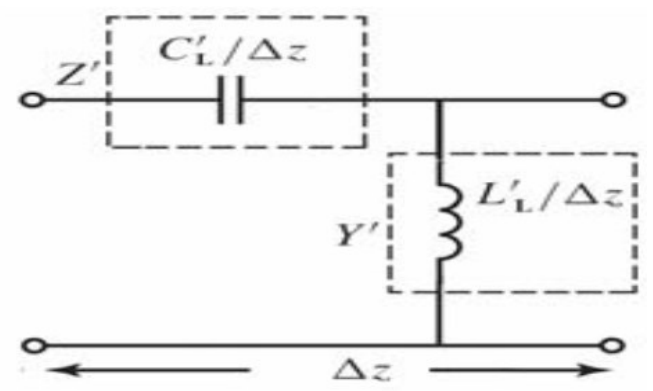

(b)

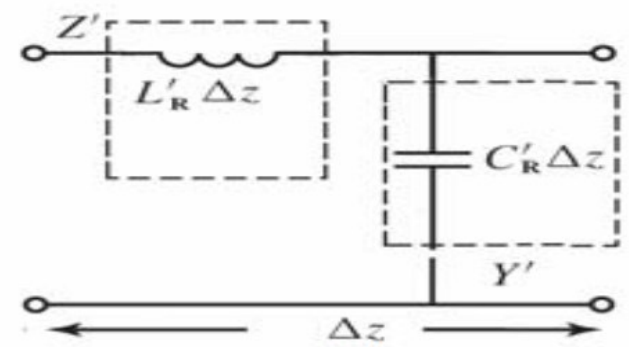

(c)

Figure 2-3: Equivalent circuit model for (a) a Homogeneous CRLH TL (b) a Homogeneous LH transmission line and (c) a Homogeneous RH transmission line [3] 
The PRH TL, which consists of a series inductance and a shunt capacitance, and the PLH TL consists of a series capacitance and a shunt inductance. The PLH TL in reality does not exist because of the parasitic series inductance and the shunt capacitance. Consequently, the CRLH TL is more common than the PLH. The complex propagation constant $\gamma$ of the transmission line can be expressed by [3][4]:

$\gamma=\alpha+\mathrm{j} \beta=\sqrt{\mathrm{Z}^{\prime} \mathrm{Y}^{\prime}}$

where $\gamma$ is the complex propagation constant, $\alpha$ is the attenuation constant, $\beta$ is the phase propagation constant and the parameters $\mathrm{Z}^{\prime}$ and $\mathrm{Y}^{\prime}$ are the impedance and the admittance per-unit length of the transmission line. In the case of the CRLH transmission line, and from Figure 2-3-a, $Z^{\prime}$ and $Y^{\prime}$ can be written as [3][4][13]:

$Z^{\prime}(\omega)=j \omega L_{R}^{\prime}+\frac{1}{j \omega C_{L}^{\prime}}$
$Y^{\prime}(\omega)=j \omega C_{R}^{\prime}+\frac{1}{j \omega L_{L}^{\prime}}$

substitute (2.8) and (2.9) into (2.7), and assuming a lossless dielectric material $(\alpha=0)$, one obtains [3]:

$\beta(\omega)=s(\omega) \sqrt{\omega^{2} L^{\prime}{ }_{R} C_{R}^{\prime}+\frac{1}{\omega^{2} L_{L}^{\prime} C_{L}^{\prime}}-\left(\frac{L_{R}^{\prime}}{L_{L}^{\prime}}+\frac{C_{L}^{\prime}}{C_{\prime}^{\prime}}\right)}$

where

$\mathrm{S}(\omega)=\left\{\begin{array}{l}-1 \text { if } \omega<\min \left(\frac{1}{\sqrt{\mathrm{L}_{\mathrm{R}} \mathrm{C}_{\mathrm{L}}}}, \frac{1}{\sqrt{\mathrm{L}_{\mathrm{L}} \mathrm{C} / \mathrm{R}}}\right) \text { LH range } \\ +1 \text { if } \omega>\max \left(\frac{1}{\sqrt{\mathrm{L}_{\mathrm{R}} \mathrm{C}_{\mathrm{L}}}}, \frac{1}{\sqrt{\mathrm{L}_{\mathrm{L}} \mathrm{C}{ }_{\mathrm{R}}}}\right) \text { RH range }\end{array}\right.$

The phase constant $\beta(\omega)$ can be purely real or purely imaginary, when $\beta$ is purely real, the electromagnetic fields will pass the CRLH transmission line without loss. If $\beta$ is pure- 
ly imaginary, then the CRLH is in a stop-band range, because $\gamma=\mathrm{j} \beta$ is a real number, for the RH transmission line, the phase constant is given by [3][4][14]:

$\beta_{\mathrm{R}}=\omega \sqrt{\mathrm{L}_{\mathrm{R}}^{\prime} \mathrm{C}_{\mathrm{R}}^{\prime}}$

and, for the LH transmission line, the phase constant is given by:

$\beta_{\mathrm{L}}=-\frac{1}{\omega \sqrt{\mathrm{L}_{\mathrm{L}} \mathrm{C}^{\prime} \mathrm{L}}}$

Based on (2.10), (2.12), and (2.13), the dispersion diagrams for PRH TL, PLH TL, and CRLH TL are shown in Figure 2-4, this graph can be also found in [3] [4] [5] and [7][9]. The $\omega$ vs. $\beta$ diagram is called the dispersion diagram, where the group velocity and the phase velocity can be obtained from the following relations:

$v_{\mathrm{g}}=\frac{\partial \omega}{\partial \beta}$

$v_{\mathrm{P}}=\frac{\omega}{\beta}$

where $v_{\mathrm{g}}$ is the group velocity $(\mathrm{m} / \mathrm{s})$, and $v_{\mathrm{P}}$ is the phase velocity $(\mathrm{m} / \mathrm{s})$.

Thus, for the pure right handed transmission line (PRH TL), the group velocity and the phase velocity have the same direction because $v_{\mathrm{g}} v_{\mathrm{P}}>0$, whereas, the group velocity and the phase velocity for the pure left handed transmission line (PLH TL) have the opposite directions because the $v_{\mathrm{g}} v_{\mathrm{P}}<0$ [3]. 


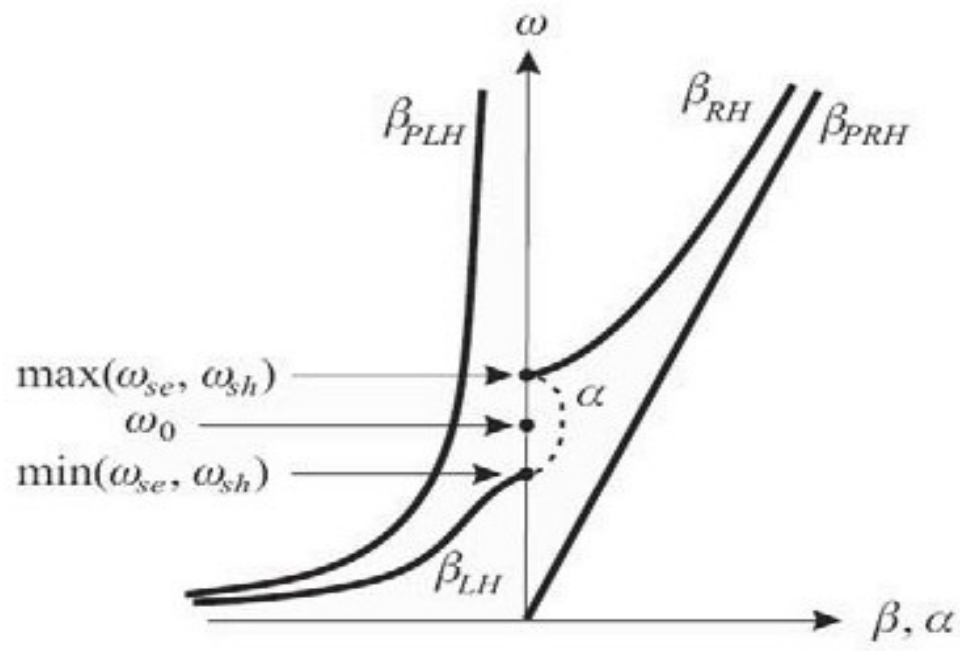

Figure 2-4: Dispersion and attenuation diagram of the CRLH TL for energy propagation along the $+\mathrm{z}$ direction $\left(v_{\mathrm{g}}>0\right)[3]$

The meaning of the symbols is as follows: $\beta_{\mathrm{RH}}$ is the phase constant of the RH range of the CRLH TL; $\beta_{\mathrm{LH}}$ is the phase constant of the LH range of the CRLH TL; $\beta_{\mathrm{PRH}}$ is the phase constant of the pure RH TL; $\beta_{\text {PLH }}$ is the phase constant of the pure LH TL [3].

The series and shunt resonances of the CRLH TL are frequently dissimilar; this is called the unbalanced case. In contrast, if the series and shunt resonances of the CRLH TL are equal, it is called the balanced case [3]. Thus

$\mathrm{L}^{\prime} \mathrm{R} \mathrm{C}^{\prime} \mathrm{L}=\mathrm{L}^{\prime} \mathrm{L} \mathrm{C}^{\prime} \mathrm{R}=\mathrm{L}^{\prime} \mathrm{C}^{\prime}$

The phase constant $\beta$ can be simplified to:

$\beta=\beta_{\mathrm{R}}+\beta_{\mathrm{L}}=\omega \sqrt{\mathrm{L}_{\mathrm{R}}^{\prime} \mathrm{C}_{\mathrm{R}}^{\prime}}-\frac{1}{\omega \sqrt{\mathrm{L}_{\mathrm{L}} \mathrm{C}_{\mathrm{L}}}}$

In the balance case, when $\beta=0$, the transition point from LH to RH occurs at $\omega_{\mathrm{o}}$ as follows [3]: 
$\omega_{\mathrm{o}}=\frac{1}{\sqrt[4]{\mathrm{L}_{\mathrm{R}} \mathrm{C}_{\mathrm{R}} \mathrm{L}_{\mathrm{L}} \mathrm{C}_{\mathrm{L}}}}=\frac{1}{\sqrt{\mathrm{L}^{\prime} \mathrm{C} \prime}}$

if $\omega$ goes to 0 then $\beta \rightarrow \beta_{L}$, and if $\omega$ goes to $+\infty$ then $\beta \rightarrow \beta_{R}$. Figure $2-4$-a shows the equivalent circuit of the balanced CRLHTL, and Figure 2-5-b shows a dispersion diagram [3]-[10]-[13].

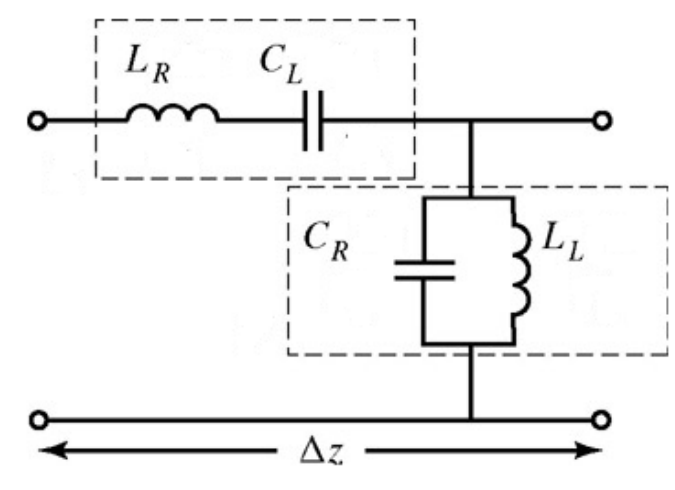

(a)

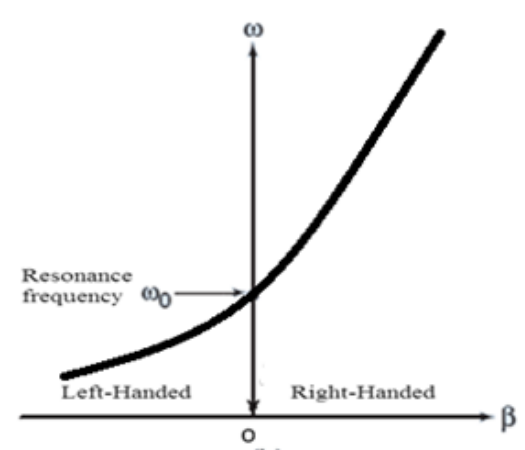

(b)

Figure 2-5: Balance case (a) Simplified equivalent circuit model (b) Dispersion diagram showing seamless LH to RH transition [3]

From the (2.17), we can observe that $\beta$ is at all times a real number. As a result, the propagation constant $\gamma=\mathrm{j} \beta$ is always purely imaginary. In this case, the stop band disappears. Even at the value of $\omega_{0}$, where $\beta=0$, the $v_{g} \neq 0$, so the electromagnetic wave is not stopped [4].

$\mathrm{Z}_{\mathrm{R}}=\sqrt{\frac{\mathrm{L}_{\mathrm{R}}^{\prime}}{\mathrm{C}_{\mathrm{R}}^{\prime}}}$

the characteristic impedance of the CRLH TL can be written as:

$\mathrm{Z}_{0}=\mathrm{Z}_{\mathrm{L}}=\mathrm{Z}_{\mathrm{R}}$

The phase constant in a material is usually defined as:

$\beta=\omega \sqrt{\mu \varepsilon}$ 
where $\mu$ is the permeability $\mathrm{H} / \mathrm{m}$ and $\varepsilon$ is the permittivity $\mathrm{F} / \mathrm{m}$.

Comparing (2.23) with (2.7) for the lossless condition $(\alpha=0)$, then:

$-\omega^{2} \mu \varepsilon=Z^{\prime} Y^{\prime}$

and the transmission line's impedance $\mathrm{Z}_{0}=\sqrt{\mathrm{Z}^{\prime} / \mathrm{Y}^{\prime}}$ can be correlated to the material intrinsic impedance as the following:

$$
\eta=\sqrt{\frac{\mu}{\varepsilon}}
$$

$\mathrm{Z}_{0}=\eta$ or $\frac{\mathrm{Z}^{\prime}}{\mathrm{Y}^{\prime}}=\frac{\mu}{\varepsilon}$

where $\mathrm{Z}^{\prime}$ and $\mathrm{Y}^{\prime}$ are the impedance and the admittance per-unit length of the transmission line, $\eta$ is the material intrinsic impedance, $\mu$ is the material permeability (Henry per meter $\mathrm{H} / \mathrm{m}$ ) and $\varepsilon$ is the material permittivity (Farad per meter $\mathrm{F} / \mathrm{m}$ ).

As a result, the corresponding permeability and the permittivity of the CRLHTL can be written as:

$$
\begin{aligned}
& \mu=\frac{Z^{\prime}}{j \omega}=L_{R}^{\prime}-\frac{1}{\omega^{2} C_{L}^{\prime}} \\
& \varepsilon=\frac{Y^{\prime}}{j \omega}=C_{R}^{\prime}-\frac{1}{\omega^{2} L_{L}^{\prime}}
\end{aligned}
$$




\subsection{Chapter Summary}

The Composite Right Left Handed Metamaterial is a new synthetic material that has unique properties of negative permittivity and negative permeability. The backward wave (BW) will occur in CRLH Metamaterial, i.e. (the group velocity $V_{g}$ and the phase velocity $V_{p}$ have the reverse direction). The underlying properties of propagation of the composite right left handed materials, such as Negative Refraction Index (NRI) has been discussed.

The basic mathematical design equations on Composite Right Left Handed (CRLH) transmission lines as well as the circuit model of the metamaterial transmission line have been presented. From the previous reference studies, the CRLH transmission line has been used in various microwave applications to develop a low-cost and a reduced-size device such as a Branch Line Coupler (BLC), which is used as a front-end-circuit in the microwave systems. 


\section{Chapter 3}

\section{Design of Metamaterial Transmission Line using LTCC Technology}

Introduction

In this chapter, the design and the fabrication of a Composite Right Left Handed Transmission Line (CRLHTL) are presented. Firstly, the concept of CRLHTL is introduced concisely. Secondly, the circuit models, which facilitate the analysis of CRLHTL are discussed. The fabrication process using Low Temperature Co-fire Ceramic (LTCC) technology is discussed. In addition, the CRLHTL is not used to implement any example in this thesis. However, as an application, a new CRLHTL is demonstrated in order to fit in the example of a branch line coupler in Chapter 4.

The artificial CRLHTL can be built using capacitors and stub inductors [13], and can be implemented by cascading microstrip unit cells, which is compatible with the microwave monolithic integrated circuit (MMICs) technologies [3].

In theory, to analyze the microstrip unit cell based CRLHTL properties, the equivalent circuit parameters must be known. The circuit model and the layout of the CRLHTL unit cell are shown in Figure 3.1 (a) and (b). 


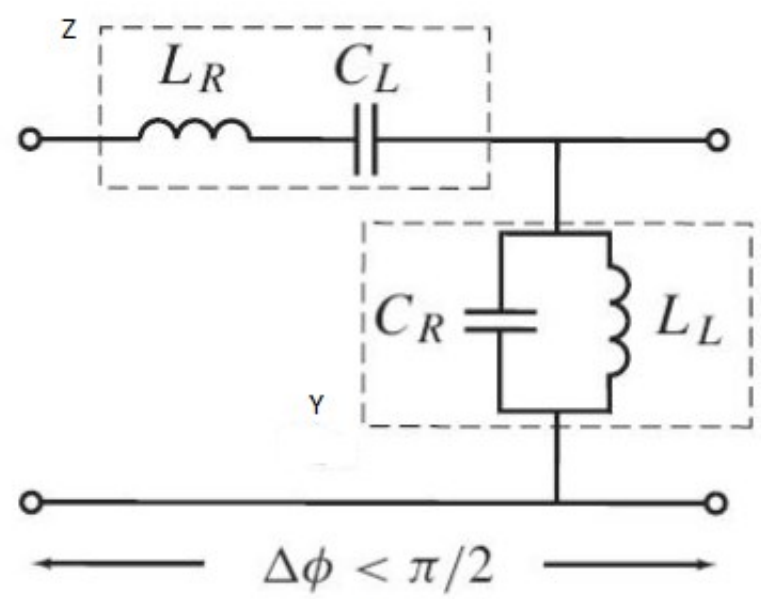

(a)

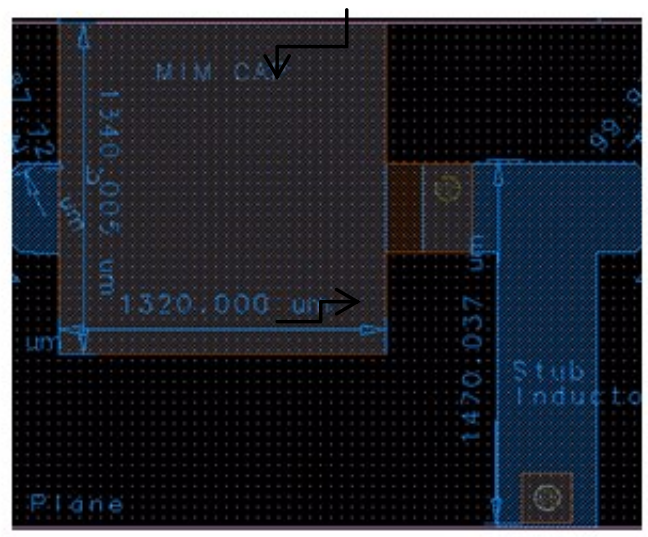

(b)

Figure 3-1: (a) Lumped element model for CRLHTL, (b) Layout of the CRLHTL.

Figure 3-1-a shows the lumped element model for the real composite right left handed transmission line. It is mapped to the layout illustrated in Figure 3-1-b. The layout is consisting of a series of a MIM capacitor and a shunt-stub inductor shorted to ground by Via, as in [3]- [10]-[17]. The series $\mathrm{C}_{\mathrm{L}}$ is implemented as a MIM (metal-insulator-metal) capacitance and the shunt inductance $\mathrm{L}_{\mathrm{L}}$ as a short-circuited stub.

In the layout structure, the parasitic elements are unavoidable and it is a portion of any layout circuit. For example, in the layout shown in Figure 3-1-b, the internal structure of a transmission line resembles the equivalent circuit shown in Figure 3-1-a, which consists of right-handed properties and left-handed properties connected together to form the composite right left handed transmission line. The layout unit cell consists of the MIM capacitors and a shunt stub inductor. The principal for this design is such that (a) the $\mathrm{C}_{\mathrm{L}}$ and $\mathrm{L}_{\mathrm{L}}$ come from the capacitance of the parallel plate and the inductance of the shunt stub, (b) the capacitance between the strip metal and the ground plane is responsible for $C_{R}$ and (c) the 
inductance along the via connected between metal-layer-2 and metal-layer-1 contributes to $\mathrm{L}_{\mathrm{R}}$.

Component designers always try to minimize the parasitic elements, but are unable to eliminate them. The parasitic elements are often modeled as lumped components in equivalent circuits. For instance, the most common parasitic elements seen in this design of work are the inductance and the capacitance of the unit cell shown in Figure 3-1-b. These elements will form the inductor $L_{R}$ and the capacitor $C_{R}$ in Figure 3-1-a, to act as a resonant circuit at some frequency. However, discontinuity due to vias, in a transmission line can also introduce a parasitic element that affects the signal transmission.

The reason is, left-handed materials in our discussion are homogeneous, and onedimensional transmission lines can model them. To fabricate the left-handed transmission line, the inductors and capacitors are loaded in the right-handed medium. As the wave propagates in the left-handed TL, the intrinsic right-handed effect cannot be avoided and a real left-handed structure includes the $\left(\mathrm{L}_{R}\right.$ and $\left.\mathrm{C}_{\mathrm{R}}\right)$ contributions in addition to the $\left(\mathrm{L}_{\mathrm{L}}\right.$ and $\mathrm{C}_{\mathrm{L}}$ ) reactance, as explained in [53].

In this chapter, the dispersion diagram of the fabricated TL and the simulated TL shows a zero degree shift at the operating frequency of $5 \mathrm{GHz}$, and a 90 degree shift at $3.2 \mathrm{GHz}$. This transmission line was initially mistakenly designed for a resonant frequency of $5 \mathrm{GHz}$ rather than the 90 degrees frequency at $5 \mathrm{GHz}$, hence the 90 degrees frequency ended up at 3.2 GHz. Therefore, the CRLHTL is designed at the operating frequency of $\mathrm{f}_{\mathrm{o}}=3.2 \mathrm{GHz}$, and characteristic impedance of $50 \Omega$. The transmission line was fabricated on LTCC ceramic substrate, with a dielectric constant of 7.1 and substrate thickness of $127 \mu \mathrm{m}$. 
Table 3.1 shows the physical properties for the stack-up of LTCC technology, and Table 3-2 shows the electrical parameters for the same stack-up.

Table 3-1: Physical parameters of the ceramic substrate of LTCC tape system [2]

\begin{tabular}{|l|l|}
\hline Physical Property & Value \\
\hline Substrate thickness for one layer & $127 \mu \mathrm{m}$ \\
\hline Permittivity & $\varepsilon \mathrm{r}=7.1 \pm 0.1$ \\
\hline Shrinkage (x,y) & 9.1 \\
\hline Shrinkage (z) & 11.8 \\
\hline Dissipation Factor & $4.7^{*} 10-3$ \\
\hline TCE $\left(23^{0}-300^{0}\right)$ & $4.4 \mathrm{ppm} / \mathrm{K}$ \\
\hline Therm. Conductivity & $4.6 \mathrm{~W} / \mathrm{mK}$ \\
\hline With thermal vias & 10 to $25 \mathrm{~W} / \mathrm{mK}$ \\
\hline Roughness & $0.52 \mu \mathrm{m}$ \\
\hline
\end{tabular}

Table 3-2: Electrical parameters of the ceramic substrate of LTCC tape system [2]

\begin{tabular}{|l|l|}
\hline Electrical Property & Value \\
\hline Dielectric constant & $7.1,+/-0.2$ \\
\hline Loss tangent & 0.0010 \\
\hline Insulation resistance, $(\mathrm{Ohm})$ & $\left(10^{12}\right)$ \\
\hline Breakdown voltage, $(\mathrm{kv} / 25 \mu \mathrm{m})$ & $\geq 1100$ \\
\hline
\end{tabular}


The LTCC technology can be applied in electronic circuits as a competitive and a cost effective substrate technology to integrate the passive and active components in different layers, Figure 3-2 shows a diagram of the structure of the LTCC layers connected with via [15].

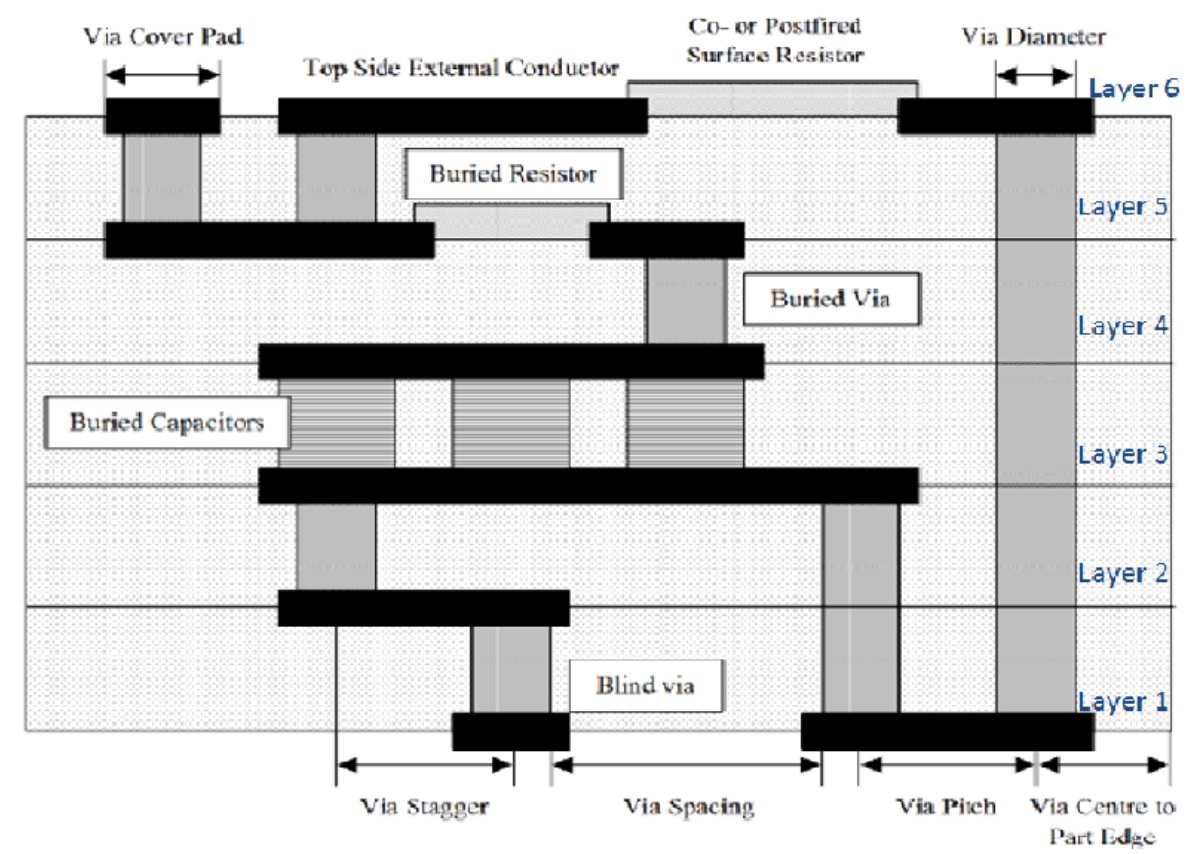

Figure 3-2: Physical structure for the LTCC with 6-Metal-Layers [2]

\subsection{Basic models}

The equivalent circuit model of a conventional right-handed transmission line (RHTL) is shown in Figure 3-3. It consists of series inductors and shunt capacitor, of which the dimensions are much less than the wavelength of the operating frequency. It is well known that many important characteristics, such as phase velocity, and dispersions characteristics could be obtained from the circuit model in Figure 3-3 as shown in Figure 3-4[3] [4]. 


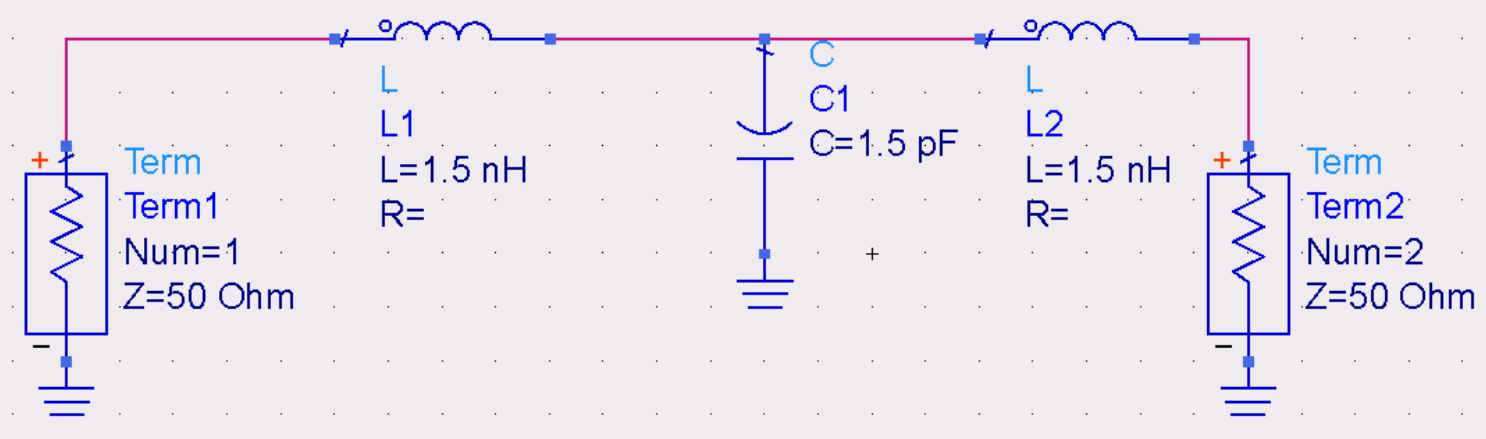

Figure 3-3: Pure right-handed transmission line (PRHTL)

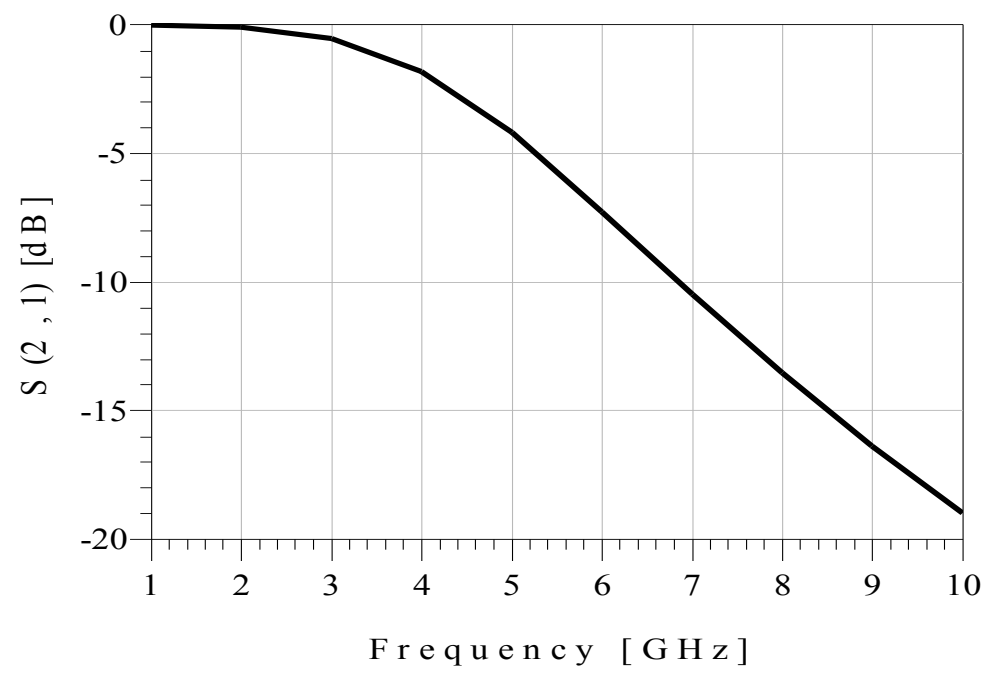

Figure 3-4: Response of the PRHTL showing passband up to $4.2 \mathrm{GHz}$

Figure 3-4 shows the frequency response of the transmission line, the result shows a passband filter up to $4.5 \mathrm{GHz}$.

On the other hand, the equivalent circuit model of a left-handed transmission line (LHTL) is shown in Figure 3-5. All series inductors in the right-handed transmission line model are 
replaced by capacitors in the left-handed transmission line model, and all shunt capacitors are substituted by inductors. It is an ideal model, which does not exist in nature [3] [4].

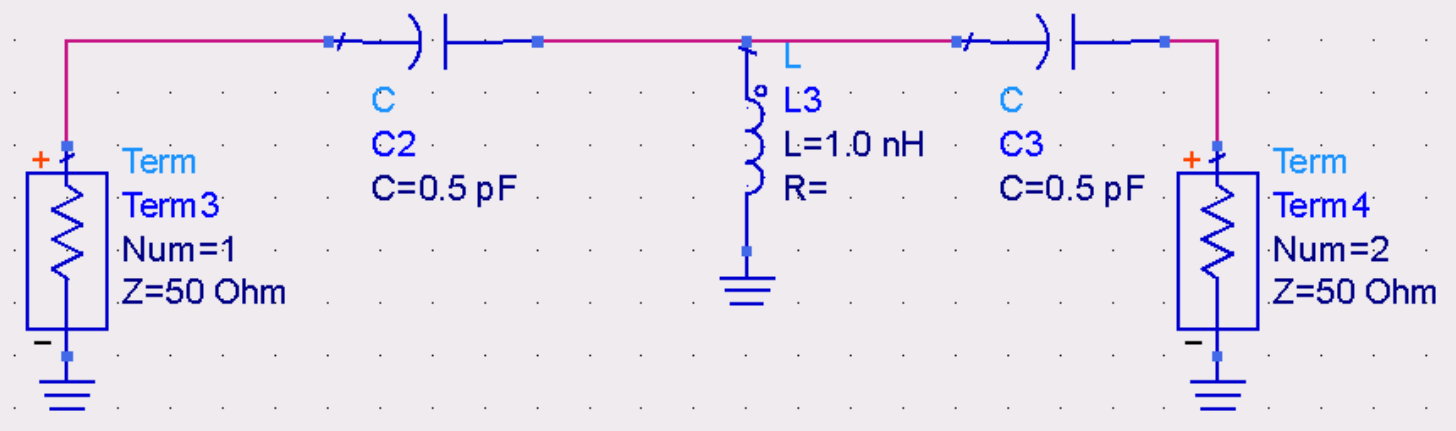

Figure 3-5: Pure left-handed transmission line (PLHTL)

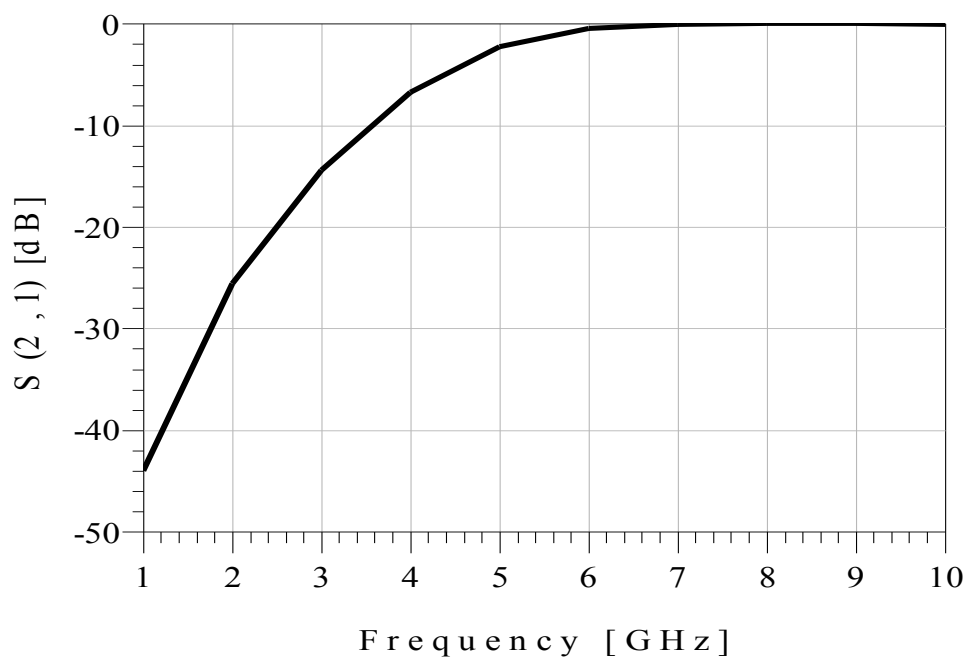

Figure 3-6: Response of the PLHTL showing pass band from about $5 \mathrm{GHz}$

From this diagram, it can be seen that from $5 \mathrm{GHz}$ onwards there is a passband behavior, which agreed well with frequency response in Figure 3-6. From the unit cell analysis, pla- 
nar structure analogy to such a lumped element circuit is a backward radiating structure throughout the passband starting from $5 \mathrm{GHz}$ [3].

Figure 3-7 shows a composite right left-handed transmission line model. It is more suitable than the left-handed transmission line circuit model, because of the parasite series inductance and shunt capacitance cannot be avoided in nature. It consists of series resonators $L_{R}$ and $\mathrm{C}_{\mathrm{L}}$ and shunt resonators $\mathrm{C}_{\mathrm{R}}$ and $\mathrm{L}_{\mathrm{L}}$. This CRLHTL circuit model is a combination of left-handed and right-handed transmission line. At low frequency, $\mathrm{C}_{\mathrm{L}}$ and $\mathrm{L}_{\mathrm{L}}$ are dominant and the CRLHTL shows left-handed characteristics. At high frequency, $L_{R}$ and $C_{R}$ are dominant and the CRLHTL shows right-handed characteristics [3] [4].

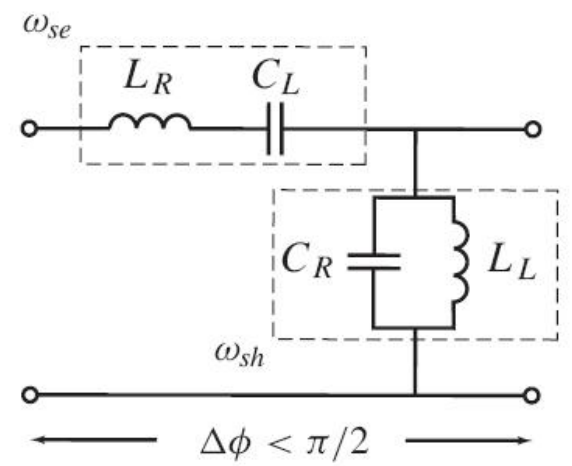

Figure 3-7: Equivalent circuit model of CRLHTL

There is no band-gap between left-handed and right-handed regions, if a so-called balanced condition is satisfied [3]. The balanced condition is

$\mathrm{L}_{\mathrm{L}} \mathrm{C}_{\mathrm{R}}=\mathrm{L}_{\mathrm{R}} \mathrm{C}_{\mathrm{L}}$

which implies the series and shunt LC resonators have the same resonant frequency transition frequency $\left(\omega_{0}\right)$. The characteristic impedance of the CRLHTL is 
$\mathrm{Z}_{\mathrm{E}}=\mathrm{Z}_{\mathrm{L}}=\mathrm{Z}_{\mathrm{R}}$

where $\mathrm{Z}_{\mathrm{L}}=\sqrt{\frac{\mathrm{L}_{\mathrm{L}}}{\mathrm{C}_{\mathrm{L}}}}$ and $\mathrm{Z}_{\mathrm{R}}=\sqrt{\frac{\mathrm{L}_{\mathrm{R}}}{\mathrm{C}_{\mathrm{R}}}}$ are the PLH and PRH characteristic impedances which are frequency independent with the homogenous transmission line approach. The cut-off frequencies of the composite right/left-handed transmission line is [3]

$$
\omega_{c L}=\omega_{R}\left(\sqrt{1+\frac{\omega_{L}}{\omega_{R}}}-1 \text { and } \omega_{c R}=\omega_{R}\left(\sqrt{1+\frac{\omega_{L}}{\omega_{R}}}+1\right.\right.
$$

where $\omega_{\mathrm{L}}=1 / \sqrt{\mathrm{L}_{\mathrm{L}} \mathrm{C}_{\mathrm{L}}}$ and $\omega_{\mathrm{R}}=1 / \sqrt{\mathrm{L}_{\mathrm{R}} \mathrm{C}_{\mathrm{R}}}$ are the resonant frequencies of the left-handed and the right-handed LC circuit, respectively. The transition frequency can be written as $\omega_{0}^{2}=\omega_{c L} \omega_{c R}=\omega_{L} \omega_{R}=1 / \sqrt{L_{L} L_{R} C_{R} C_{L}}$.

Considering the CRLHTL design with periodic elements, there are four parameters, specifically $L_{L}, C_{L}, L_{R}$, and $C_{R}$. When the balanced condition is applied, there are only three independent parameters left. Once two cut-off frequencies and the characteristic impedance $Z_{\mathrm{E}}$ (matching to the system impedance $\mathrm{Z}_{0}$ ) are fixed, a unique composite right left handed configuration is determined [3]

$\omega_{0} L_{R}=\frac{1}{\omega_{0} C_{L}}=\frac{\omega_{0}}{\omega_{c R}-\omega_{c L}} 2 Z_{0}$

$\omega_{0} C_{R}=\frac{1}{\omega_{0} C_{L}}=\frac{\omega_{0}}{\omega_{c R}-\omega_{c L}} 2 / Z_{0}$. 


\subsection{Overview of Transmission Line Theory}

To extract the parameters of the CRLHTL, scattering matrices have to be obtained for the capacitors and the inductors. However, the scattering matrices cannot be obtained directly, it requires a reference plane shift method to calculate the scattering matrices [11][20][21].

\subsubsection{ABCD Matrix and S-Parameters}

The homogeneous CRLHTL can be understood in the structure of a ladder network circuit as shown in Figure 3-8. One of the main advantages of $\mathrm{ABCD}$ parameters in microwave circuit analysis is the simplicity in cascaded circuits, (especially the periodic N-cell ladder network), which is valid if the coupling is minimal between various sections of the circuit [3][13][22].

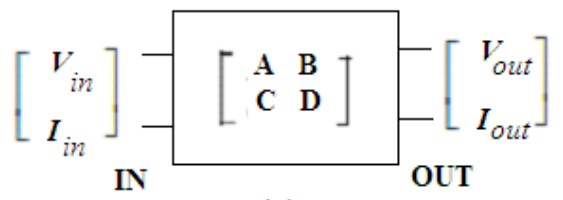

(a)

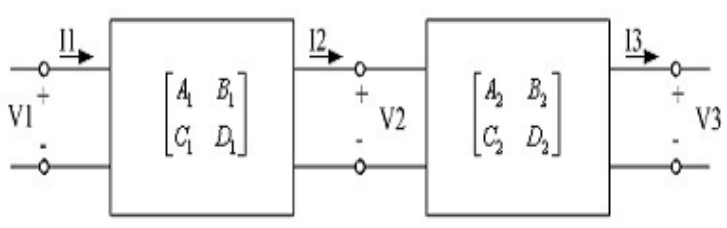

(b)

Figure 3-8: The ABCD matrix (a) Two port network (b) Cascade connection of two-port network [3] [4]

Considering the two-port network in Figure 3-8-a, it relates the input current and voltage to the output current and voltage in the following method: 
$\left[\begin{array}{l}V_{\text {in }} \\ I_{\text {in }}\end{array}\right]=\left[\begin{array}{ll}A & B \\ C & D\end{array}\right]\left[\begin{array}{l}V_{\text {out }} \\ I_{\text {out }}\end{array}\right]$

The same methodology can be applied to cascade connection of N-cell two-port networks shown in Figure 3-8-b:

$\left[\begin{array}{ll}A_{N} & B_{N} \\ C_{N} & D_{N}\end{array}\right]=\prod_{k=1}^{N}\left[\begin{array}{ll}A_{k} & B_{k} \\ C_{k} & D_{k}\end{array}\right]=\left[\begin{array}{cc}A_{k} & B_{k} \\ C_{k} & D_{k}\end{array}\right]^{N}$ For identical cell

That is, the $\left[\mathrm{A}_{N} \mathrm{~B}_{N} C_{N} D_{N}\right]$ matrix is equal to the product of the ABCD matrices for the individual cell, $\left[\mathrm{A}_{\mathrm{K}} \mathrm{B}_{\mathrm{K}} \mathrm{C}_{\mathrm{K}} \mathrm{D}_{\mathrm{K}}\right][1][4]$.

\subsubsection{CRLH TL Analysis}

Figure 3-9 shows the equivalent circuit model of CRLHTL consisting of a series impedance $\mathrm{Z}$ and a shunt admittance $\mathrm{Y}$, which is repeatedly used in research and its ABCD matrix format $[1][3][6][20][21]$.

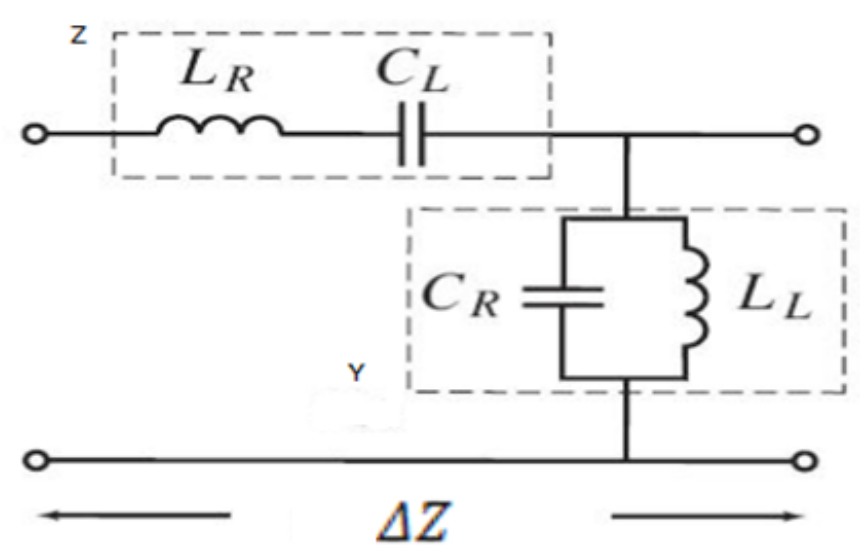

Figure 3-9: Equivalent circuit model of CRLHTL [3]

The ABCD matrix of the structure above can be written as follows:

$\left[\begin{array}{ll}\mathrm{A} & \mathrm{B} \\ \mathrm{C} & \mathrm{D}\end{array}\right]_{\mathrm{CRLH}}=\left[\begin{array}{ll}1 & Z \\ 0 & 1\end{array}\right]\left[\begin{array}{ll}1 & 0 \\ \mathrm{Y} & 1\end{array}\right]=\left[\begin{array}{cc}1+\mathrm{ZY} & \mathrm{Z} \\ \mathrm{Y} & 1\end{array}\right]$ 
and the complex propagation constant $\gamma$ can be expressed by [3]

$\gamma=\alpha+\mathrm{j} \beta=\sqrt{\mathrm{ZY}}$

where $\gamma$ is the complex propagation constant, $\alpha$ is the attenuation constant, $\beta$ is the phase propagation constant and the parameters $\mathrm{Z}$ and $\mathrm{Y}$ are the impedance and the admittance per-unit length of the CRLHTL, respectively, can be written as [3]

$Z(\omega)=j\left(\omega L_{R}-\frac{1}{\omega C_{L}}\right) ; Y(\omega)=j\left(\omega C_{R}-\frac{1}{\omega L_{L}}\right)$

By substituting (3-5) into (3-4), and assuming a lossless dielectric material $(\alpha=0)$, one obtains [3]

$\beta(\omega)=s(\omega) \sqrt{\omega^{2} L_{R}^{\prime} C_{R}^{\prime}+\frac{1}{\omega^{2} L_{L}^{\prime} C_{L}^{\prime}}-\left(\frac{L_{R}^{\prime}}{L_{L}^{\prime}}+\frac{C_{R}^{\prime}}{C_{L}^{\prime}}\right)}$

where

$\mathrm{S}(\omega)=\left\{\begin{array}{l}-1 \text { if } \omega<\min \left(\frac{1}{\sqrt{\mathrm{L}_{\mathrm{R}}^{\prime} \mathrm{C}_{\mathrm{L}}^{\prime}}}, \frac{1}{\sqrt{\mathrm{L}_{\mathrm{L}}^{\prime} \mathrm{C}_{\mathrm{R}}^{\prime}}}\right) \text { LH range } \\ +1 \text { if } \omega>\max \left(\frac{1}{\sqrt{\mathrm{L}_{\mathrm{R}}^{\prime} \mathrm{C}_{\mathrm{L}}^{\prime}}}, \frac{1}{\sqrt{\mathrm{L}_{\mathrm{L}}^{\prime} \mathrm{C}_{\mathrm{R}}^{\prime}}}\right) \mathrm{RH} \text { range }\end{array}\right.$

The dispersion relation can be obtained by Kirchoff's law and applying the periodic boundary conditions (PBCs) of the Bloch-Floquet theorem, thus [3] [25]

$\beta(\omega)=\frac{1}{\Delta \mathrm{Z}} \cos ^{-1}\left(1+\frac{\mathrm{ZY}}{2}\right)$

where $\Delta \mathrm{Z}<<\lambda \mathrm{g}$ at least $\left(\Delta \mathrm{Z}<\frac{\lambda \mathrm{g}}{4}\right)$

where $\lambda \mathrm{g}$ represents the guided wavelength and and $\Delta \mathrm{z}$ is typically equal the average unit cell size $\mathrm{p}$. 
From the $\mathrm{ABCD}$ matrix equation (3-3), the equivalent S-parameters of the network with termination ports impedance $\mathrm{Z}_{0}$ can be derived as the following [1][3][4]:

$$
\left[\begin{array}{ll}
S_{11} & S_{12} \\
S_{21} & S_{22}
\end{array}\right]=\frac{1}{A+\frac{B}{Z_{o}}+C Z_{o}+D}\left[\begin{array}{cc}
A+\frac{B}{Z_{o}}-C Z_{o}-D & 2(A D-B C) \\
2 & A+\frac{B}{Z_{o}}-C Z_{o}-D
\end{array}\right]
$$

The characteristic impedance of CRLH TL in the balanced case (i.e., $\mathrm{L}_{\mathrm{R}} \mathrm{C}_{\mathrm{L}}=\mathrm{L}_{\mathrm{L}} \mathrm{C}_{\mathrm{R}}$ ) can be written as:

$\mathrm{Z}_{\mathrm{c}}=\mathrm{Z}_{\mathrm{L}}=\sqrt{\frac{\mathrm{L}_{\mathrm{L}}}{\mathrm{C}_{\mathrm{L}}}}=\mathrm{Z}_{\mathrm{R}}=\sqrt{\frac{\mathrm{L}_{\mathrm{R}}}{\mathrm{C}_{\mathrm{R}}}}$

if the CRLH TL has the same characteristic impedance of the termination ports impedance $\mathrm{Z}_{0}$, the phase shift of the unit cell can be derived as the following:

$\emptyset_{\mathrm{c}}=-\arctan \left(\frac{\omega_{\mathrm{L}}\left[\left(\frac{\omega}{\omega_{\mathrm{O}}}\right)^{2}-1\right]\left[\left(1-\left(\frac{\mathrm{x}}{4}\right)-\left(\frac{1}{\omega}\right)\right)\right]}{\omega(2-\mathrm{x})}\right)$

where

$\mathrm{x}=\left(\frac{\omega}{\omega_{\mathrm{R}}}-\frac{\omega_{\mathrm{L}}}{\omega}\right)^{2}, \quad \omega_{\mathrm{R}}=\frac{1}{\sqrt{\mathrm{L}_{\mathrm{R}} \mathrm{C}_{\mathrm{R}}}}, \omega_{\mathrm{L}}=\frac{1}{\sqrt{\mathrm{L}_{\mathrm{L}} \mathrm{C}_{\mathrm{L}}}}$

$\omega_{0}=2 \pi \mathrm{f}_{0}=\omega_{\mathrm{sh}}=\omega_{\mathrm{se}}=\frac{1}{\sqrt{\mathrm{L}_{\mathrm{L}} \mathrm{C}_{\mathrm{R}}}}$

and $\omega$ is in $\frac{\mathrm{rad}}{\mathrm{s}}$.

The series and shunt resonance frequencies are given by

$$
\begin{aligned}
& \omega_{s e}=1 / \sqrt{L_{R} C_{L}} \mathrm{rad} / \mathrm{s} \\
& \omega_{s h}=1 / \sqrt{L_{L} C_{R}} \mathrm{rad} / \mathrm{s}
\end{aligned}
$$


At the balance case the CRLH characteristics impedance is obtained explicitly by the following:

$Z_{c}=Z_{L} \sqrt{\frac{\left(\omega / \omega_{s e}\right)^{2}-1}{\left(\omega / \omega_{s h}\right)^{2}-1}}$

And

$\mathrm{Z}_{\mathrm{L}}=\sqrt{\frac{\mathrm{L}_{\mathrm{L}}}{\mathrm{C}_{\mathrm{L}}}}$

$\mathrm{Z}_{\mathrm{R}}=\sqrt{\mathrm{L}_{\mathrm{R}} / \mathrm{C}_{\mathrm{R}}}$

The CRLH TL exhibits interesting properties in the particular case where the series and shunt resonant frequencies are equal [3]

$\omega_{s e}=\omega_{s h}$ or equivalently, $\mathrm{Z}_{\mathrm{L}}=\mathrm{Z}_{\mathrm{R}}$

In contrast, in the balanced case $\left(\omega_{s e}=\omega_{s h}=\omega_{o}\right)$, and the zero $\omega_{s e}$ and the pole $\omega_{s h}$ in the characteristic impedance cancel each other suppressing resonance effect [3]. As a consequence, the characteristic impedance becomes a frequency-independent quantity, where $\mathrm{Z}_{\mathrm{c}}=\mathrm{Z}_{\mathrm{R}}=\mathrm{Z}_{\mathrm{L}}$

This means that the balanced condition allows matching over an infinite bandwidth [3].

Consider the CRLHTL shown in Figure 3-10, N=1, $\mathrm{L}_{\mathrm{L}}=0.02 \mathrm{nH}, \mathrm{L}_{\mathrm{R}}=2.5 \mathrm{nH}$ and $\mathrm{C}_{\mathrm{L}}=2.5$ $\mathrm{pF}$ and $\mathrm{C}_{\mathrm{R}}=0.04 \mathrm{pF}$. where $\mathrm{N}$ is the number of unit cells.

The dispersion diagram of the transmission line is shown in Figure 3-12, and the phase shift of (S21) is 90 degrees at the frequency point of $3.2 \mathrm{GHz}$ as shown in Figure 3-11-a. The scattering parameters show that the $\mathrm{S} 11$ has less than $-20 \mathrm{~dB}$ at the resonance frequency, and the TL has a bandwidth close to $3 \mathrm{GHz}$ at S11 of $-10 \mathrm{~dB}$. 33 


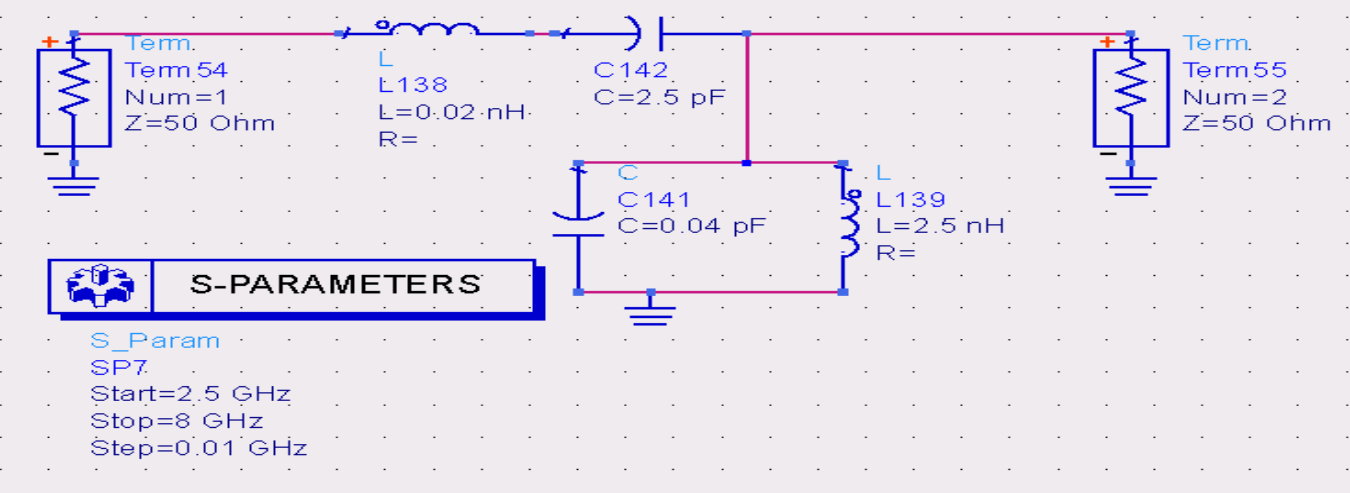

Figure 3-10: Schematic diagram of the CRLHTL

Figure 3-11-a and 3-11-b shows the phase response and the scattering parameters of the CRLHTL, respectively. The return loss $\mathrm{S}_{11}$ at $3.2 \mathrm{GHz}$ is about $-6.5 \mathrm{~dB}$ and it is perfect at the resonance point. The insertion loss $\mathrm{S}_{21}$ shows a reasonable value of the power transmitted from port 1 to port $2 \mathrm{~S}_{21}=-0.25 \mathrm{~dB}$ at the frequency of $3.2 \mathrm{GHz}$, and $0 \mathrm{~dB}$ at the resonance point. In Figure 3-11-a, the phase shift $\left(\mathrm{S}_{21}\right)$ shows a 90 degrees phase at $3.2 \mathrm{GHz}$, and shows 0 degrees phase shift at resonance frequency of $5 \mathrm{GHz}$.

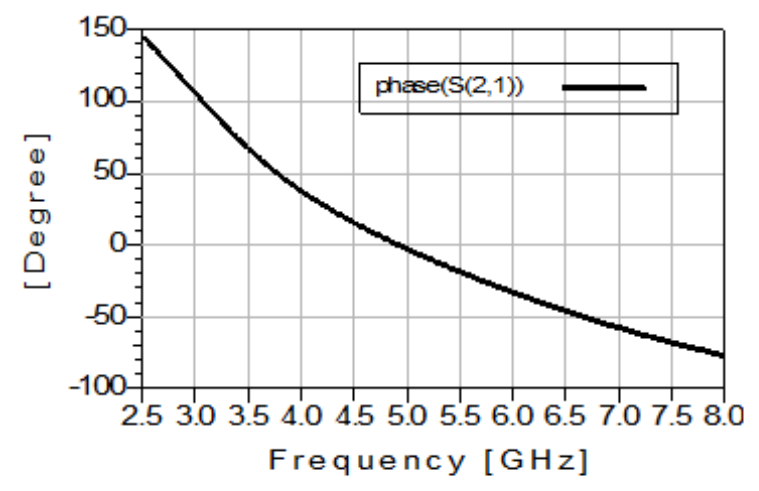

(a)

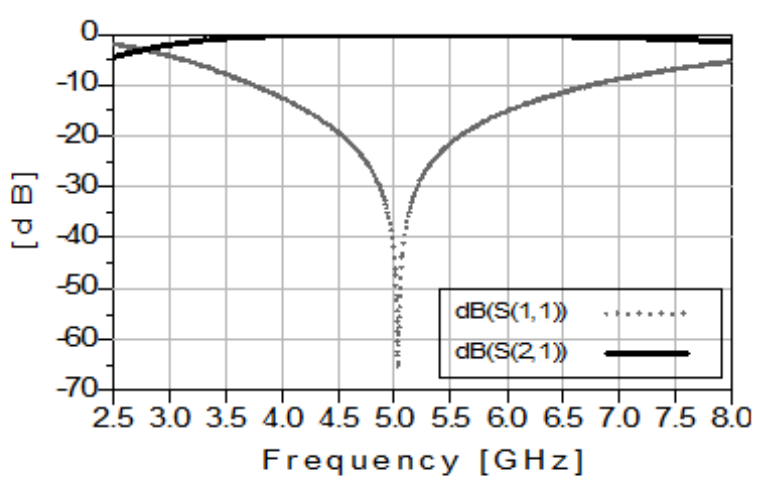

(b)

Figure 3-11: Simulated (a) Phase response, (b) S-parameters of the TL model shown in Figure 3-10 
In Figure 3-12, the dispersion diagram obtained using the unit cell analysis is given in Figure 3.10. Although the CRLHTL has both a left-handed range and a right-handed range, the dispersion curve in each of these ranges significantly differs from that of the Pure Left Handed (PLH) and Pure Right Handed (PRH) structures, respectively, because of the combined effects of left-handed and right-handed contributions at all frequencies [3].

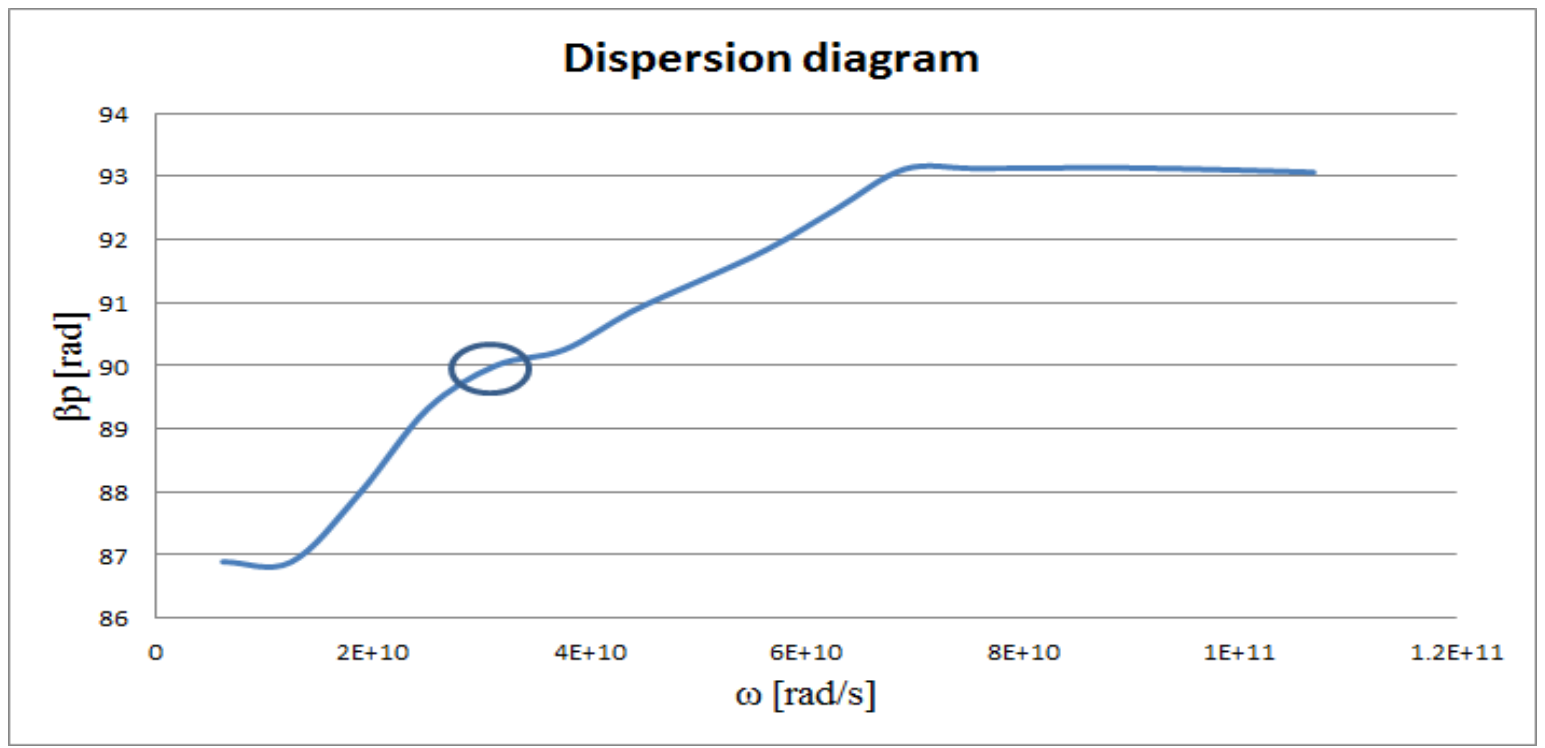

Figure 3-12: Dispersion diagram of the TL model shown in Figure 3-11

\subsection{Layout of the CRLH Transmission Line}

The initial step in the proposed design methodology is to synthesize the values of the stub inductor and MIM capacitor based on the design frequency and the characteristic impedance, followed by a simulation of the layout design using the microstrip transmission line. The following equations were used to synthesize the element values based on the frequency and characteristic impedance [3]. 
$\mathrm{L}=\frac{Z_{o}}{2 \pi \mathrm{f} \tan (\beta \mathrm{p})}$

where $\mathrm{L}$ is the inductance value $(\mathrm{nH}), \mathrm{Z}_{\mathrm{o}}$ is the characteristic impedance $(\Omega), \mathrm{f}$ is the design frequency, $\beta$ is the electrical phase constant radians/meter and $p$ is the length of the transmission line.

$\mathrm{C}=\frac{\varepsilon_{\mathrm{o}} \varepsilon_{\mathrm{r}} \mathrm{WLe}}{\mathrm{h}}$

where $\mathrm{C}$ is the capacitance value $(\mathrm{pF}), \mathrm{W}$ and Le are the width and the length of the parallel plate of the MIM capacitor, and $\mathrm{h}$ is the distance between the two plates.

The phase constant as a function of frequency is given by

$$
\beta=2 \pi \mathrm{f} \sqrt{\mathrm{LC}}
$$

Figure 3-13 shows the layout of the CRLH transmission line shown in Figure 3-10. The operating frequency is chosen to be $\mathrm{f}_{\mathrm{o}}=5 \mathrm{GHz}$. The signal line is implemented on the top metal layer C01_T while the ground plane of the microstrip element is implemented on C02_B. The width of the signal line is $220 \mu \mathrm{m}$ and the width of the ground plane is 2089 $\mu \mathrm{m}$. The two metal layers $\mathrm{C} 01 \_\mathrm{T}$ and $\mathrm{C} 02 \_\mathrm{T}$ form the MIM capacitor. Whereas the stubinductor formatted on one metal layer C01_T and connected through VIA to the ground. The VIA radius is $50 \mu \mathrm{m}$, the length is $127 \mu \mathrm{m}$, and the $250 \mu \mathrm{m}$ testing pad is formatted on the top layer C01_T and connected through VIA to the ground plane, the size of the CRLH transmission line without test pad is $2.183 \mathrm{~mm} \times 2.041 \mathrm{~mm}$. The measurements of the transmission line were carried out using Agilent-E8361A $10 \mathrm{MHz}$ to $67.0 \mathrm{GHz}$ vector network analyzer, and the EM simulation was performed by using an electromagnetic simulator (Agilent ADS). 


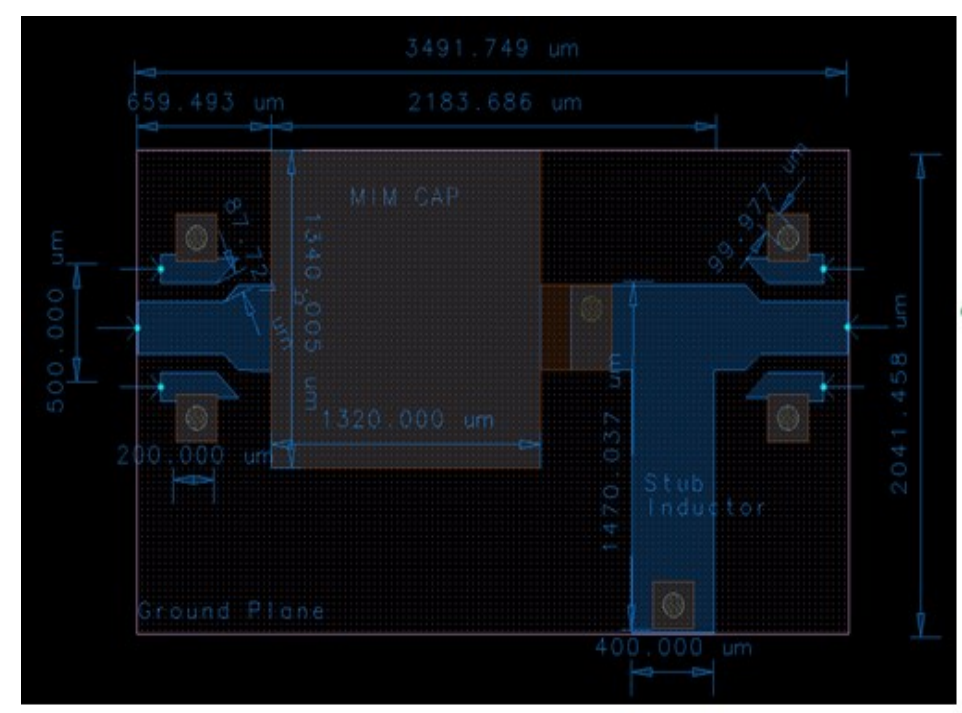

(a)

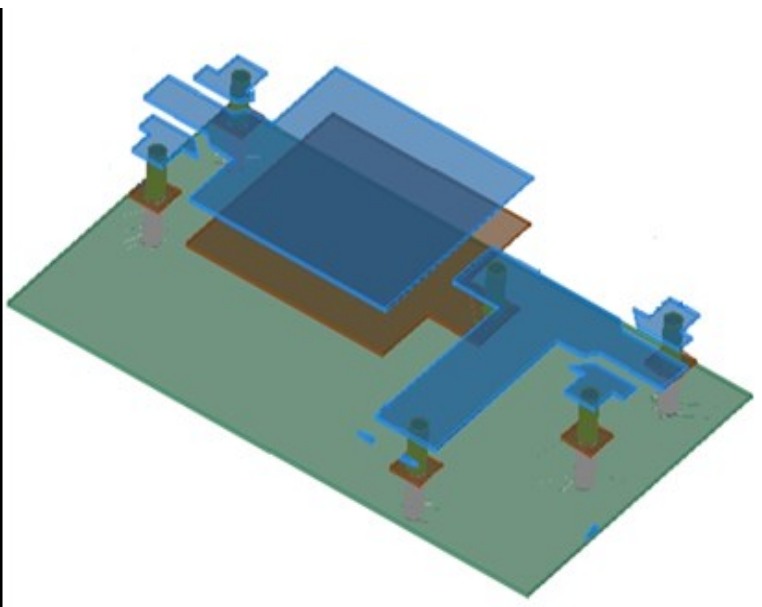

(b)

Figure 3-13: (a) Layout of the unit cell of the CRLHTL, (b) 3-D view

\subsection{Experimental and post-layout Simulation Results}

Figure 3-14 shows the manufactured LTCC wafer which consists of 3-metal- layers of DuPont 9K7 [2]. The scattering parameters have been measured for the CRLHTL inside the red-box using an Agilent E861A network analyzer, while the EM simulation performed using an electromagnetic simulator (Agilent ADS momentum). 


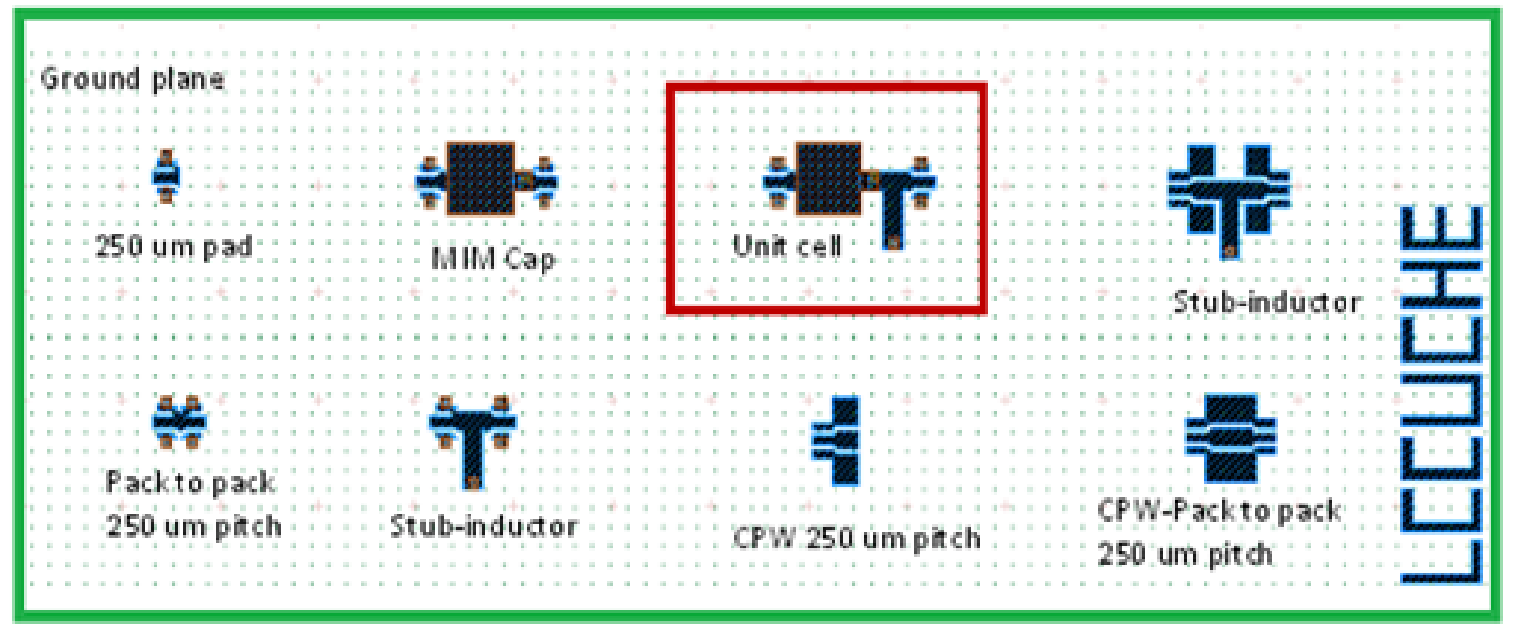

Figure 3-14: Top-view of the LTCC wafer

The phase response of the CRLHTL is plotted in Figure 3.15. From this figure, we have two frequencies at which phase is 90 degrees one was lagging and one leading at $3.2 \mathrm{GHz}$ and $8.7 \mathrm{GHz}$, respectively. This kind of circuits can be used to design dual-band (DB) components, such as a dual-band branch line coupler. Such a dual-band component therefor accomplishing the same function at two different arbitrary frequencies $\omega_{1}$ and $\omega_{2}$.

in chapter 4 of this research a branch line coupler is designed using a single band frequency and occupied a frequency bandwidth $400 \mathrm{MHz}$.

Also from this figure, at the center frequency of $5 \mathrm{GHz}$ the phase shift equal to zero, and therefore $\omega_{0}=2 \pi f_{0}$ is defined as the critical frequency or the phase origin of the CRLHTL [3]. Also it can be seen that the unit cell indeed has left handed properties over the frequency from $1-5 \mathrm{GHz}$ and right handed behavior over the frequency range of 5-10 GHz as can see in Figure 3-17. 
The essential characteristics of a CRLHTL circuit shown in Figure 3-13 can be inferred from analysis of the equivalent circuit of Figure 3-1-a. At low frequencies, $L_{R}$ and $C_{R}$ tend to be short and open, respectively, so that the equivalent circuit is essentially reduced to the series $\mathrm{C}_{\mathrm{L}}$ and shunt $\mathrm{L}_{\mathrm{L}}$ circuit, which is $\mathrm{LH}$ since it has antiparallel phase and group velocities; this LH circuit is of high-pass nature; therefore, below a certain cut-off, a LH stopband is present. At high frequencies, $\mathrm{C}_{\mathrm{L}}$ and $\mathrm{L}_{\mathrm{L}}$ tend to be short and open, respectively, so that the equivalent circuit is essentially reduced to the series $L_{R}$ and shunt $C_{R}$ circuit, which is RH since it has parallel phase and group velocities; this LH circuit is of low-pass nature; therefore, above a certain cut-off, a RH stop-band is present. In this case, the series resonance $\omega_{\text {se }}$ and shunt resonance $\omega_{\text {sh }}$ are equal, so that there is no gap exists between the LH and the RH ranges. However, this circuit designed where these resonances are balanced, that explain this gap disappears, and an infinite wavelength $(\lambda g=2 \pi /|\beta|)$ propagation is achieved at the transition frequency $\omega_{0}$. Note that, although a CRLH structure has both a LH range and a RH range, the dispersion curve Figure 3-17 in each of these ranges significantly differs from that of the PLH and PRH structures, respectively, because of the combined effects of LH and RH contributions at all frequencies [3] [4]. 


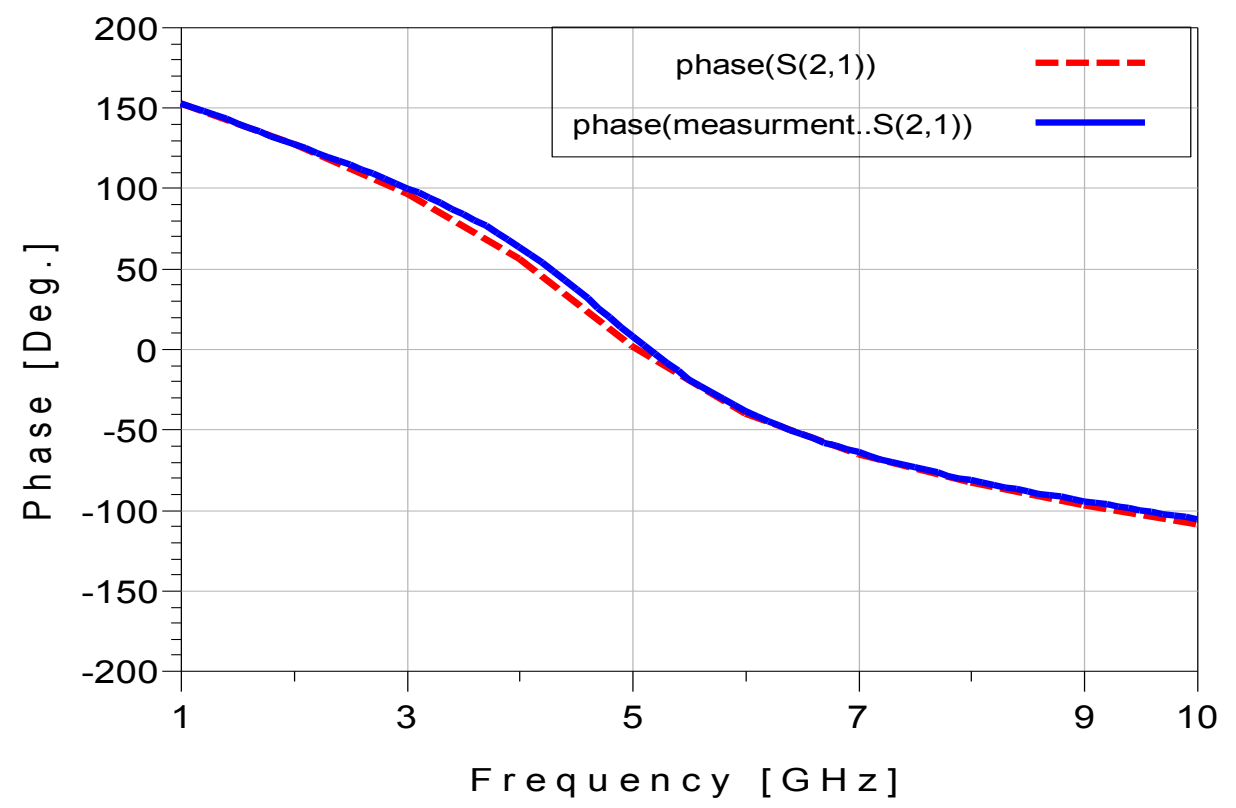

Figure 3-15: Measured and simulated of S(2,1) of the CRLHTL shown in Figure 3-13 and $3-14$

Figure 3-16-a shows the measured versus the simulated return loss magnitude response for port 1 where the results show a good agreement between the two. The measured and simulated return loss is below $-10 \mathrm{~dB}$ over the bandwidth of 4.5-5.5 GHz. Figure 3-17-b shows the power transmitted from port 1 to port 2, the measured and simulated insertion loss S21 are in good agreement, the measured and the simulated insertion loss are remain above -1.5 $\mathrm{dB}$ for the entire bandwidth range from 4.5-5.5 GHz.

Figure 3-16 shows the S-parameters of the fabricated versus simulated CRLHTL. From Figure 3-16 the S-parameters $\left(\mathrm{S}_{11}\right)$ and $\left(\mathrm{S}_{21}\right)$ of the fabricated CRLHTL match the simulated transmission line over the frequency range 1-10 GHz. The dispersion diagram of the fabricated TL shows a zero degree at the resonance frequency $5 \mathrm{GHz}$, while the same diagram shows a 90 degree at different value of frequency $3.2 \mathrm{GHz}$ as seen in Figure 3-17. Thus, this circuit becomes nearly a short circuit in series and open circuit in parallel, so as 40 
expected the match is good and the Insertion loss $\left(\mathrm{S}_{21}\right)$ is good. However, since we need to operate at 90 degrees, this circuit doesn't satisfy this condition. Also, the zero-degree phase shift is not sufficient to design a branch line coupler, where this type of coupler required a 90-degree phase shift. This transmission line was initially mistakenly designed for a resonant frequency of $5 \mathrm{GHz}$ rather than the 90 degrees frequency at $5 \mathrm{GHz}$, hence the 90 degrees frequency ended up at $3.2 \mathrm{GHz}$. In chapter 4 , the design of the branch line coupler required a 90-degree phase shift. Thus, a new design of CRLHTL will be implemented to satisfy this condition. For instance, this CRLHTL will stay since it will be considered as a useful information as part of LTCC technology evaluation.

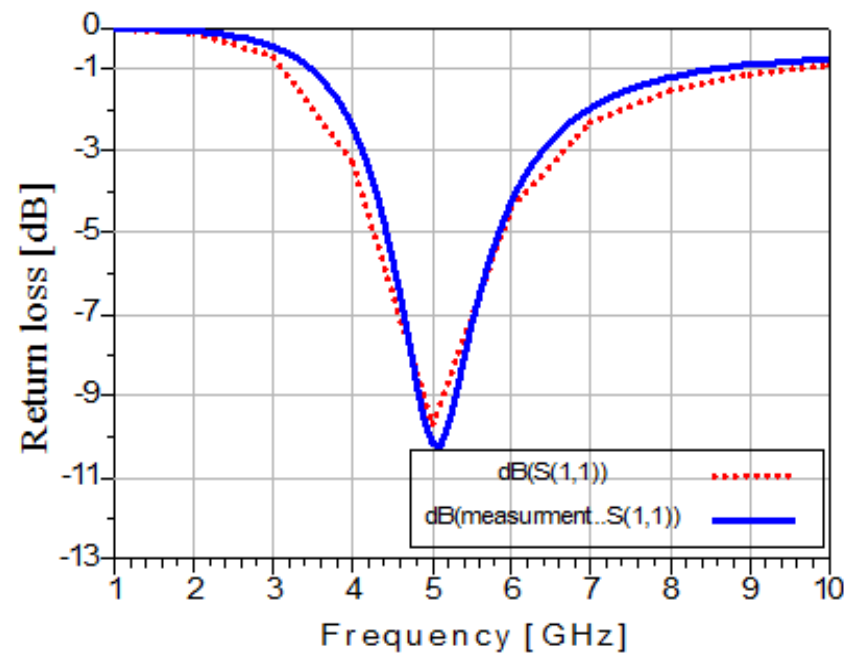

(a)

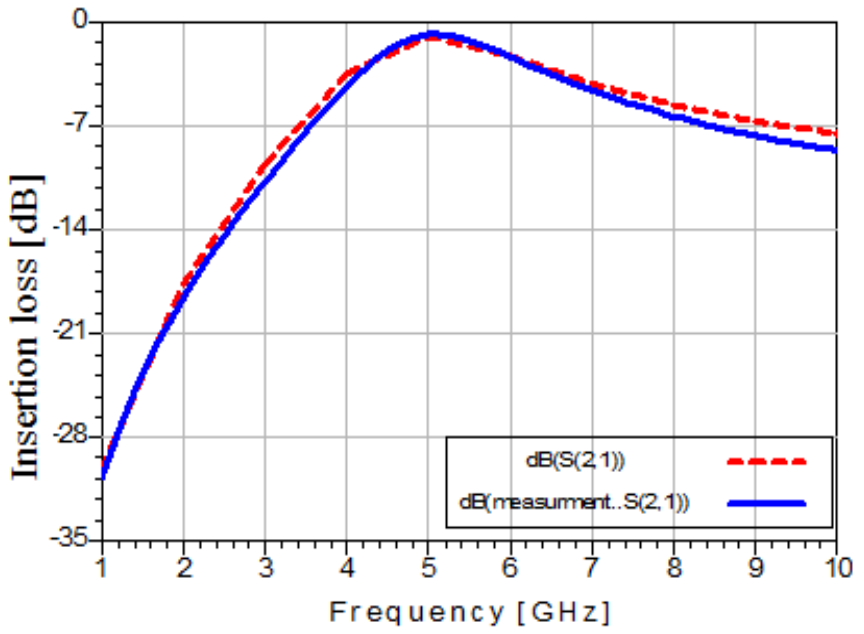

(b)

Figure 3-16: Measured and simulated (a) Return loss, (b) Insertion loss

The measured and the simulated results were compared using excel spread sheet of the SPfile for the measured TL and the S-parameters of the simulated transmission line. Figure 316 illustrates that a good agreement between the measurements and post-simulation results have been achieved, because of the Agilent (E861A) network analyzer achieves highly ac- 
curate measurements by correcting for the systematic errors in the instrument, the characteristics of cables, adapters and test fixtures.

The measurements have been taken over the frequency range of 1 to $20 \mathrm{GHz}$ using OSL (Open-Short-Load) in order to achieve better source match and load match corrections than the other calibrations methods. The Open-Short-Load requires a high-reflection standard (usually, a short or open) whose impedance does not have to be well characterized, but it must be electrically the same for both test ports. The CRLHTL calibration has been performed using a full 2-port reflectivity and transmission calibration. In this case there were 12 possible systematic errors, such as source match, directivity and reflection tracking, where the most common method for correcting these involves measuring a short, load and open standard on each of the two ports, as well as transmission between the two ports. The difference between the measured and simulated results is due to the accuracy of the ceramic substrate parameters of the LTCC technology. This difference between the measurement and the simulation results is not critical over the frequency band. Thus, the measurement and the post-layout simulation results are in close agreement over the bandwidth of (4.5-5.5 $\mathrm{GHz})$.

Figure 3-17 shows the dispersion diagram obtained using the fabricated and the simulated CRLHTL. The CRLHTL has both a left-handed range and a right-handed range, the dispersion curve in each of these ranges significantly differs from that of the PLH and PRH structures, because of the combined effects of left-handed and right-handed contributions at all frequencies. 


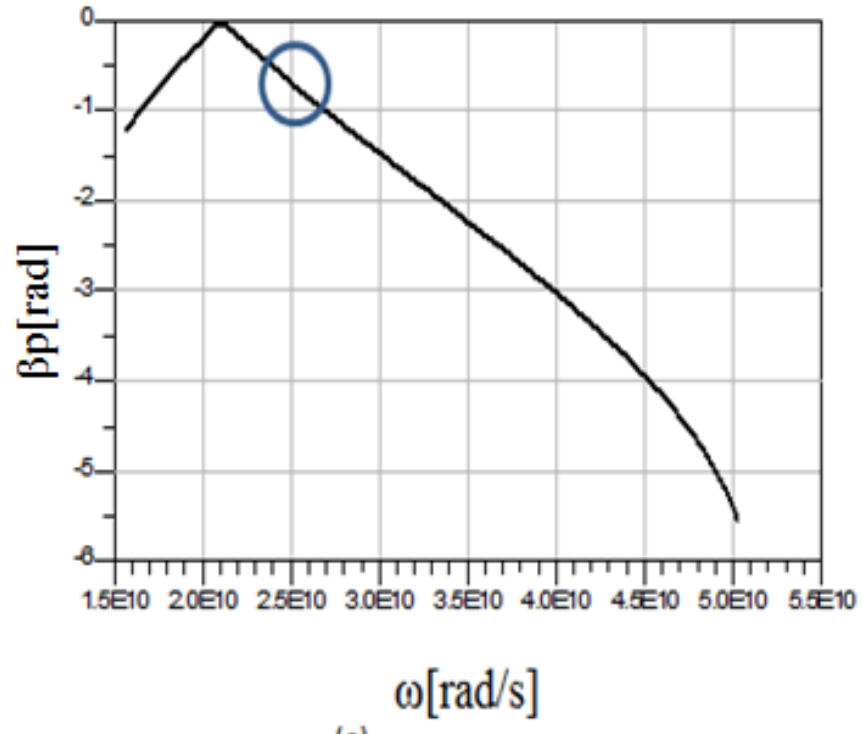

(a)

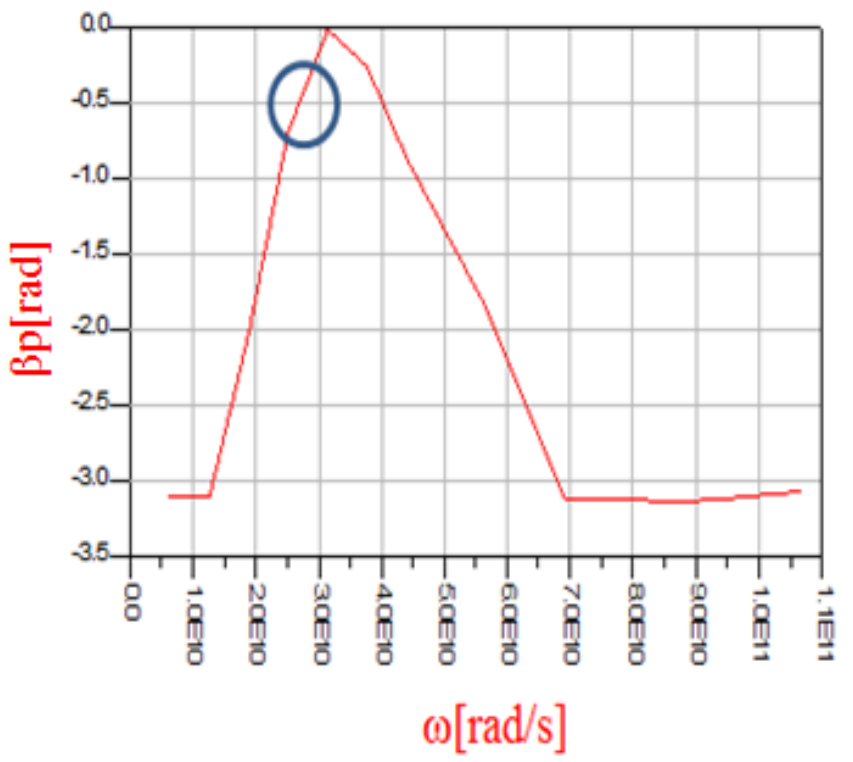

(b)

Figure 3-17: Dispersion diagram of the (a) Measured CRLHTL shown in Figure 3-10, (b)

Simulated CRLHTL shown in Figure 3-14 


\subsection{Chapter Summary}

The main objective of this chapter is to discuss the design of the CRLHTL and the fabrication process of its layout using LTCC technology. The CRLHTL is constructed using MIM-capacitors and shorted stub-inductors. The CRLHTL is fabricated using three metal layers, and tested over the bandwidth of 1-10 GHz. The dispersion diagram of the transmission line shows a negative propagation phase constant below $5 \mathrm{GHz}$ and a positive propagation phase constant above the center frequency of $5 \mathrm{GHz}$.

The conventional TL (RHTL) is represented by a series inductor $\left(\mathrm{L}_{\mathrm{R}}\right)$ and a shunt capacitance $\left(\mathrm{C}_{\mathrm{R}}\right)$, implying the use of a low pass topology. By interchanging the position of the inductor and capacitor, the resulting structure is referred to as Left Handed Transmission Line (LHTL) with a high pass configuration, as shown in section 3.1.

In this LHTL, the phase and group velocities are opposite to each other. The LHTL is considered as the 1-D version of the so-called metamaterials [3]. Composite Right Left Handed (CRLH) TL represents practical LHTL, because RH parasitic effects are unavoidable when the realization of a LHTL is attempted.

In the layout structure, the parasitic elements are unavoidable and it is a portion of any layout circuit. For example, in the layout shown in Figure 3-1-b, the internal structure of a transmission line resembles the equivalent circuit shown in Figure 3-1-a, which consists of right-handed properties and left-handed properties connected together to form the composite right left handed transmission line.

The dispersion diagram of the fabricated TL and the simulated TL shows a zero degree at the operating frequency of $5 \mathrm{GHz}$, and a 90 degrees at the $3.2 \mathrm{GHz}$. This transmission line 44 
was initially mistakenly designed for a resonant frequency of $5 \mathrm{GHz}$ rather than the 90 degrees frequency at $5 \mathrm{GHz}$, hence the 90 degrees frequency ended up at $3.2 \mathrm{GHz}$. Thus, the zero-degree phase shift is not sufficient to design such applications as a branch line coupler. Thus, this transmission line can be used to design different applications at $3.2 \mathrm{GHz}$, such as antennas, notch filter and diplexer.

However, the S-parameters of the post-layout simulation results were compared with the measurement results and a suitable match was observed over the bandwidth of 1-10 GHz. This circuit does not behave like a real transmission line at $5 \mathrm{GHz}$, since the real transmission line with finite length would have finite phase shift at $5 \mathrm{GHz}$, thus, this circuit can be used instead of a short circuit at $5 \mathrm{GHz}$. 


\section{Chapter 4}

\section{Design of Branch Line Coupler Using CRLHTL}

In this chapter, a Branch Line Coupler (BLC) is designed based on the Composite Right Left Handed Transmission Line (CRLHTL). The CRLHTL, which is used to implement the BLC is different from the transmission line in Chapter 3. The difference is simply that TL in chapter 3 was designed for the wrong frequency, so in this chapter it will be redesigned to move the frequency to $4.5-5.5 \mathrm{GHz}$.

In this example, a new transmission line is designed that achieves a 90-degrees phase shift and shows a good performance over the frequency band of (4.5-5.5 GHz). In addition, the BLC is implemented on ceramic substrate of LTCC technology using 3-metal layers with a substrate thickness of $127 \mu \mathrm{m}$ and a constant dielectric of 7.1. This type of coupler has important characteristics that widely used in microwave circuits, such as power combiners and dividers, balanced mixers, image rejection mixers, and balanced amplifiers [3].

At low frequencies of the microwave band, the size of the BLC becomes larger because the wavelength is inversely proportional to the operating frequency, thus, it is needed to reduce the size of the coupler to agree with the application requirements [27].

Several techniques were used to reduce the size of the BLCs. One of these techniques is replacing each section of the conventional transmission line coupler by equivalent

CRLHTL that have characteristic impedances $Z_{o} / \sqrt{2}$ and $Z_{o}$ for the horizontal and vertical branches, respectively, which connected to form the branch line coupler [3]. 


\subsection{Conventional Branch-Line Coupler in LTCC Technology}

The principle of the conventional branch line coupler is shown in Figure 4-1, the input power is split equally between the coupled and the direct ports with a $90^{\circ}$ phase shift difference, and the fourth (isolated) port is terminated by a 50-ohm load [27].

The quarter wavelength $\lambda / 4$ transmission lines are used in the distributed design of the conventional coupler. The design method selected for this example uses the CRLHTL presented in the previous chapter.

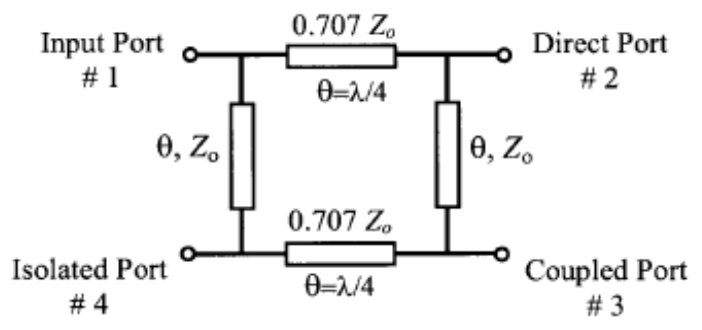

Figure 4-1: A conventional branch-line coupler [27]

Figure 4-1 shows the conventional BLC consists of four quarter wavelengths spaced at $\lambda / 4$, the horizontal branch line impedance is $\left(\frac{z_{o}}{\sqrt{2}}\right)$ and the vertical branch line impedances is $\mathrm{Z}_{0}$, where $\mathrm{Z}_{\mathrm{o}}$ is the characteristic impedance $=50 \Omega[30][31]$.

If ports 2 and 3 are properly terminated with matching impedances, nearly all the signals applied to port 1 is transmitted to the loads connected to ports 2 and 3 . In this situation, port 4 receives negligible power and is termed isolated, however, if an impedance mismatch occurs at port 2, then the signal power reflected back from port 2 will be divided proportionally between ports 1 and 4, and the power is not fed to port 3 [29]. There are 47 
many parameters describing the performance of BLC networks such as coupling factor, directivity, phase difference between the output ports and isolation [29][32], as follows:

Coupling factor $=\mathrm{C}(\mathrm{dB})=10 \log \left[\frac{\mathrm{P}_{1}}{\mathrm{P}_{3}}\right]=20 \log \left|\mathrm{S}_{31}\right|$

Directivity $=\mathrm{D}(\mathrm{dB})=10 \log \left[\frac{\mathrm{P}_{3}}{\mathrm{P}_{4}}\right]=20 \log \left|\mathrm{S}_{31} / \mathrm{S}_{41}\right|$

and the isolation and transmitted power are given by:

Isolation $=\mathrm{I}(\mathrm{dB})=10 \log \left[\frac{\mathrm{P}_{1}}{\mathrm{P}_{4}}\right]$ Or $=\mathrm{D}+\mathrm{C}=-20 \log \left|\mathrm{S}_{41}\right|$

Transmitted power $=\mathrm{T}(\mathrm{dB})=10 \log \left[\frac{\mathrm{P}_{2}}{\mathrm{P}_{1}}\right]=20 \log \left|\mathrm{S}_{21}\right|$

\subsection{Modeling and Simulation of $90^{\circ}$ conventional LTCC BLC}

\subsubsection{Design Equations}

The fundamental design equations can be written as:

$$
\beta .1=\frac{\pi}{2}
$$

$\mathrm{Z}_{\text {horizontal }}=\frac{\mathrm{z}_{\mathrm{o}}}{\sqrt{2}}$

$\mathrm{Z}_{\mathrm{vertical}}=\mathrm{Z}_{\mathrm{o}}$

with

$$
\begin{aligned}
& \beta . \mathrm{l}=\frac{\pi}{2} \\
& \mathrm{l}=\frac{\pi}{2 . \beta}=\frac{\pi \cdot \mathrm{c}}{2 . \omega}=\frac{\mathrm{c}}{4 . \mathrm{f}}=\frac{\lambda}{4}
\end{aligned}
$$

where $l$ is defined by a quarter wavelength, $Z_{o}$ is the characteristic impedances $=50 \Omega$.

To design a $3 \mathrm{~dB}$ coupler, by applying the design equations as the followings [3]-[6]: 
$\mathrm{Z}_{\text {horizontal }}=\frac{\mathrm{Z}_{\mathrm{o}}}{\sqrt{2}} \cdot=35.35 \Omega$

$\mathrm{Z}_{\mathrm{vertical}}=\mathrm{Z}_{\mathrm{o}}=50 \Omega$

where $\mathrm{Z}_{\mathrm{o}}$ is the characteristic impedance which is $=50 \Omega$.

\subsubsection{Simulation of Conventional BLC Using Lumped Elements}

Figure 4-2 shows the circuit design of the conventional branch line coupler using lumped elements. It consists of two horizontal lines which have impedance of $35 \Omega$ each, and the vertical lines with a $50 \Omega$ impedance each [21][35].

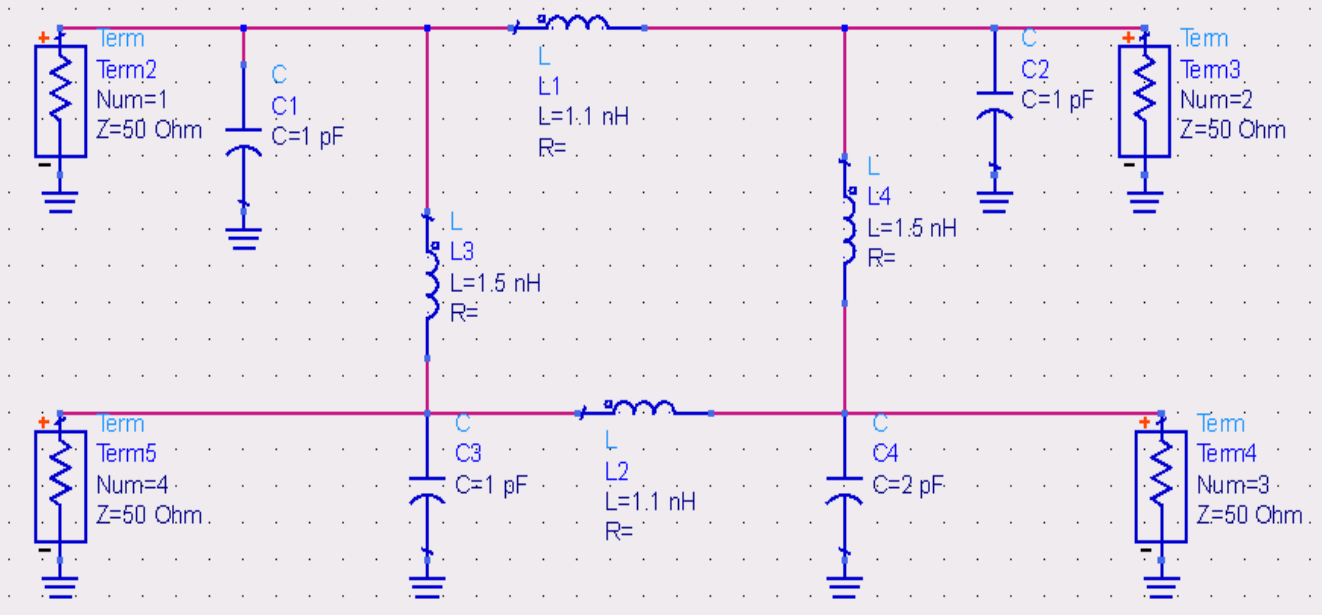

Figure 4-2: Conventional BLC uses lumped components

The values of lumped elements are obtained by equating $\mathrm{ABCD}$-matrix parameters to the ABCD-matrix of the structure. The ABCD-matrix of a lossless transmission line section of characteristic impedance $Z_{o}$ and the electrical length $\theta$ is given by [21]: 
$\left[\begin{array}{ll}\mathrm{A} & \mathrm{B} \\ \mathrm{C} & \mathrm{D}\end{array}\right]=\left[\begin{array}{cc}\cos \theta & \mathrm{j} \mathrm{Z}_{\mathrm{o}} \sin \theta \\ \frac{\mathrm{j}}{\mathrm{Z}_{\mathrm{o}}} \sin \theta & \cos \theta\end{array}\right]$

and the ABCD-matrix of any of the series arm or shunt arm networks shown in Figure 4-2 is given by [21]:

$$
\begin{aligned}
{\left[\begin{array}{ll}
A & B \\
C & D
\end{array}\right]=\left[\begin{array}{cc}
1 & 0 \\
j \omega C & 1
\end{array}\right]\left[\begin{array}{cc}
1 & j \omega L \\
0 & 1
\end{array}\right]\left[\begin{array}{cc}
1 & 0 \\
j \omega C & 1
\end{array}\right] } \\
=\left[\begin{array}{cc}
1-\omega^{2} L C & j \omega L \\
j \omega C\left(2-\omega^{2} L C\right) & 1-\omega^{2} L C
\end{array}\right]
\end{aligned}
$$

by equating the matrix elements in (4.9) and (4.10), the following results have obtained:

$$
\begin{aligned}
& \cos \theta=1-\omega^{2} \mathrm{LC} \\
& \theta=\cos ^{-1}\left(1-\omega^{2} \mathrm{LC}\right)=90 \\
& \mathrm{Z}_{\mathrm{o}} \sin \theta=\omega \mathrm{L} \\
& \frac{1}{\mathrm{Z}_{\mathrm{o}}} \sin \theta=\omega \mathrm{C}\left(2-\omega^{2} \mathrm{LC}\right) \\
& \frac{1}{\mathrm{Z}_{\mathrm{o}}}=\sqrt{\frac{2 \mathrm{C}}{\mathrm{L}}-(\omega \mathrm{C})^{2}}
\end{aligned}
$$

or

$\mathrm{L}=\frac{\mathrm{Z}_{\mathrm{o}} \sin \theta}{\omega}$

$C=\frac{1}{\omega Z_{o}} \sqrt{\frac{1-\cos \theta}{1+\cos \theta}}$

when $\theta=90^{\circ}$ then $\operatorname{Cos}(\theta)=0$, and the element values are:

$$
\begin{aligned}
& \mathrm{L}=\frac{\mathrm{Z}_{\mathrm{o}}}{\omega} \\
& \mathrm{C}=\frac{1}{\omega \mathrm{Z}_{\mathrm{o}}}
\end{aligned}
$$


The analysis does not contain any losses and any other lumped element parasitic effects. Typical lumped-element values for a $5 \mathrm{GHz}$ coupler designed for $50 \Omega$ terminal impedance are: $\mathrm{L}=1.59 \mathrm{nH}, \mathrm{C}=0.636 \mathrm{pF}$, and for the $35 \Omega$ terminal impedance $\mathrm{L}=1.11 \mathrm{nH}$, $\mathrm{C}=0.909 \mathrm{pF}$.

Figure 4-3 shows the layout of the conventional BLC constructed using microstrip TLs with a constant dielectric of 7.1 and a substrate thickness of $127 \mu \mathrm{m}$.

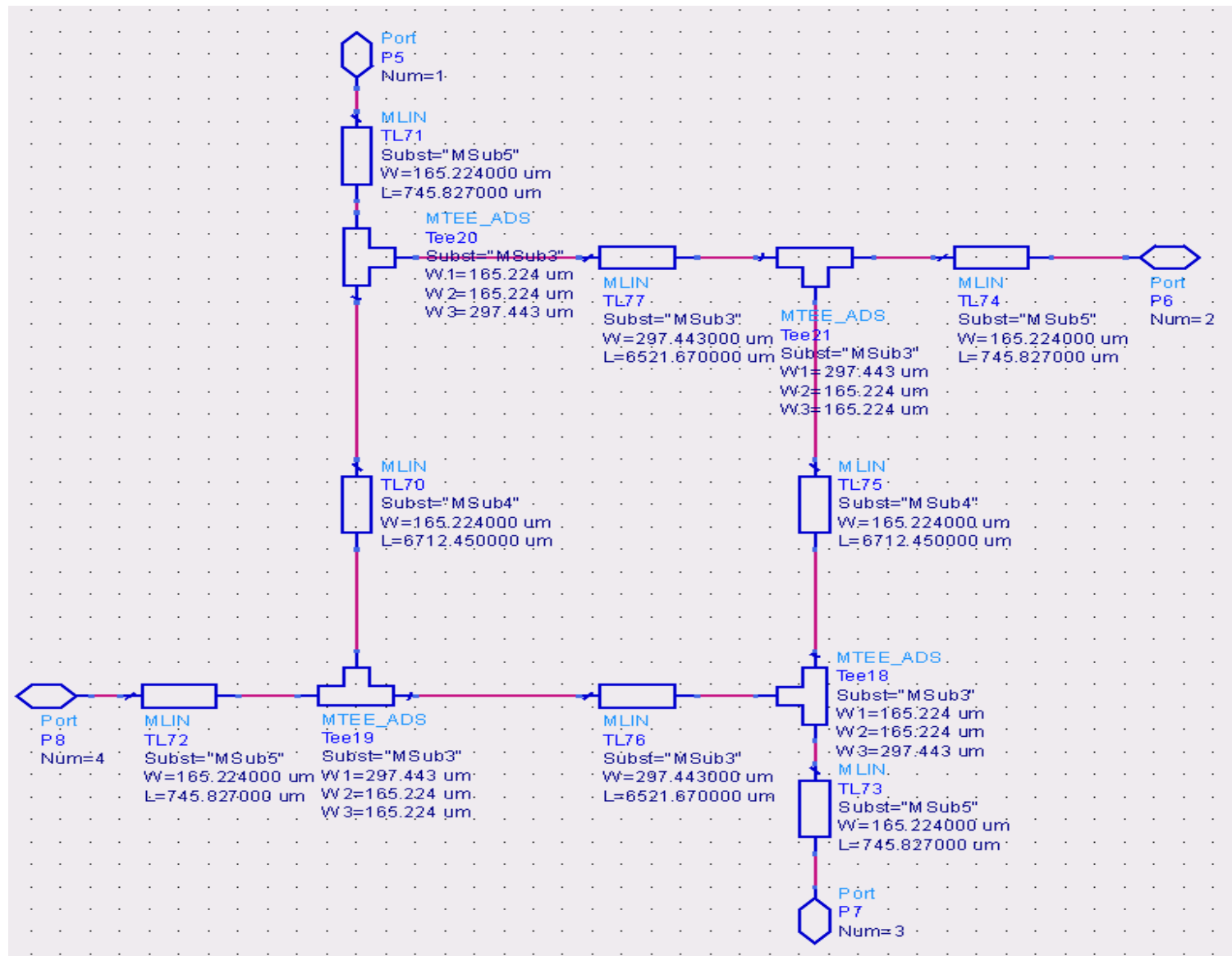

Figure 4-3: Coupler model using microstrip elements 
Figure 4-3 shows the coupler model using microstrip elements, this coupler have external transmission lines connected to each port to enable adjusting the phase between these ports. Also contains a T-junctions at the corners of the coupler, these T-junctions would affect the electrical length of the transmission line, thus it can be also adjusted by simply adjusting the lengths of the external transmission lines.

Figure 4-4-a shows the 3-dimensions view of the conventional BLC. The coupler is designed using two metal layers with a substrate thickness of $127 \mu \mathrm{m}$ and a dielectric of 7.1 . The simulated S-parameters at the schematic level of the BLC are shown in Figure 4-5-b, where the insertion loss between the input and the direct ports (S21) is about $-3 \mathrm{~dB}$ from (4.5-5.5 GHz), and the coupling between the input and the coupled ports (S31) close to -4 $\mathrm{dB}$ at $5 \mathrm{GHz}$. The amplitude imbalance between the direct and the coupled ports is shown in Figure 4-5-a. Whereas the phase difference between the direct and the coupled ports of the BLC is shown in Figure 4-5-d, where the phase difference between the output ports are 90 degrees at $5 \mathrm{GHz}$. Figure 4-5-c shows the return loss for the conventional BLC where the return losses for all ports are less than $-10 \mathrm{~dB}$ for the bandwidth $(4.5-5.5 \mathrm{GHz})$. The size of the simulated conventional BLC is $6.604 \mathrm{~mm} \times 6.778 \mathrm{~mm}$. 


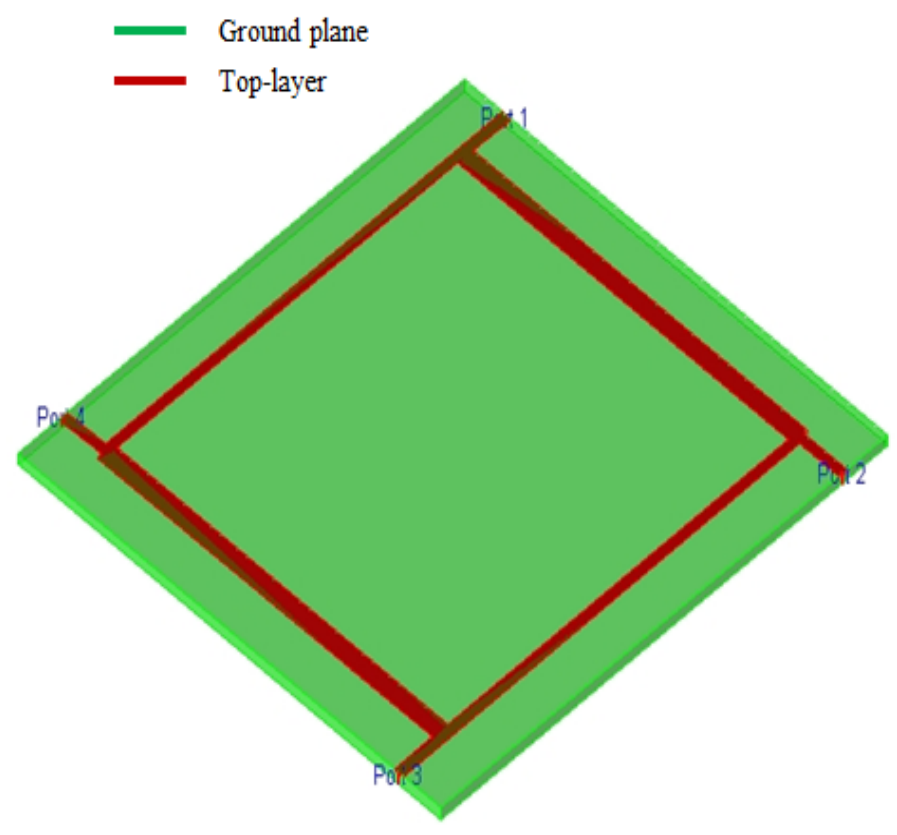

(a)

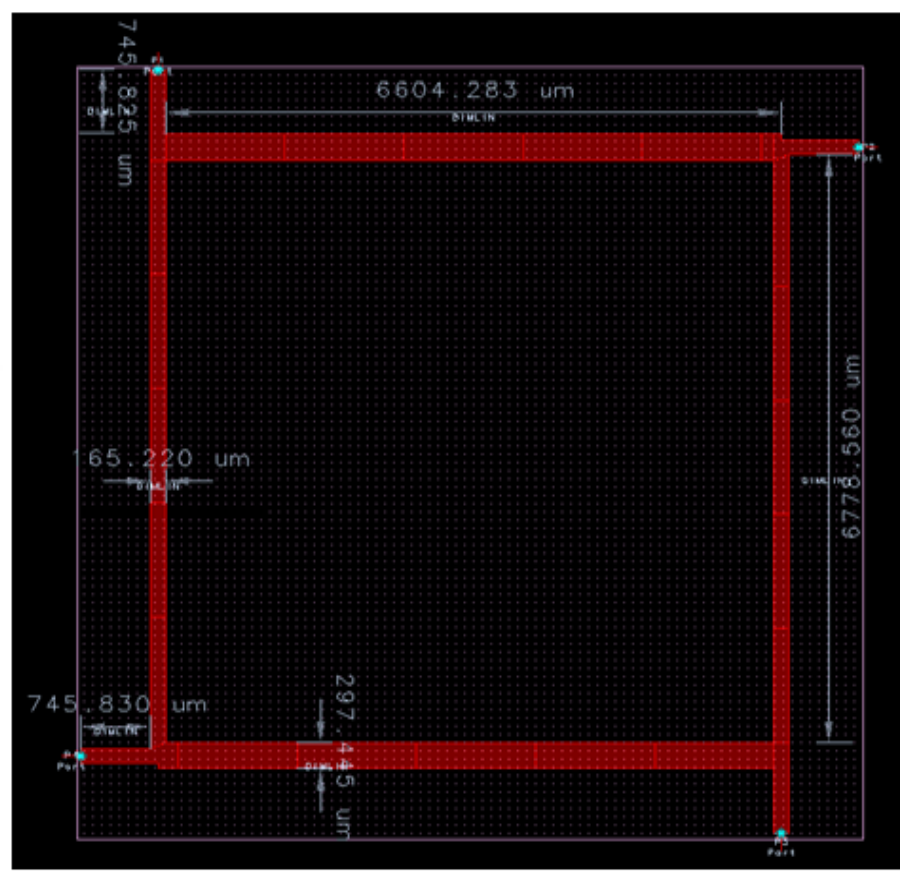

(b)

Figure 4-4: Layout of the conventional BLC (a) 3-D view, (b) Dimensions view 

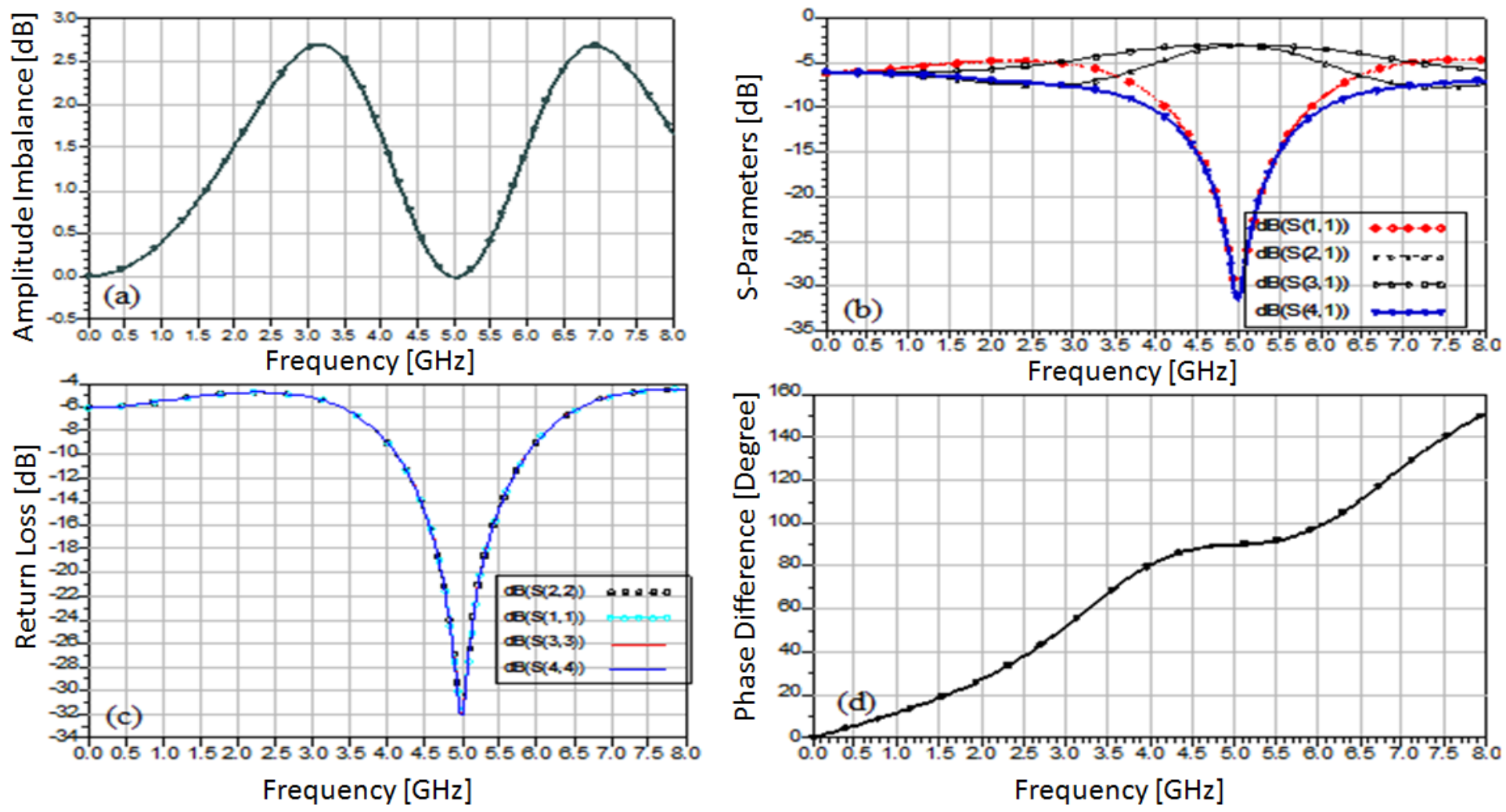

Figure 4-5: Results of the conventional BLC (a) Amplitude imbalance, (b) S-parameters, (c) Return loss, (d) Phase difference 


\subsection{Reducing the Branch Line Coupler's Size Using CRLHTL}

In this sub-section, a new transmission line is designed, which achieves a 90-degree phase shift and shows a good performance over the frequency band of $(5-5.3 \mathrm{GHz})$. The layout of the BLC implemented on ceramic substrate of LTCC technology using 3-metal layers with a substrate thickness of $127 \mu \mathrm{m}$ and a constant dielectric of 7.1 .

We will replace each section of transmission line in the conventional coupler, which has electrical length of 90 degree, by equivalent circuit of transmission line connected in series as shown in Figure 4-6.

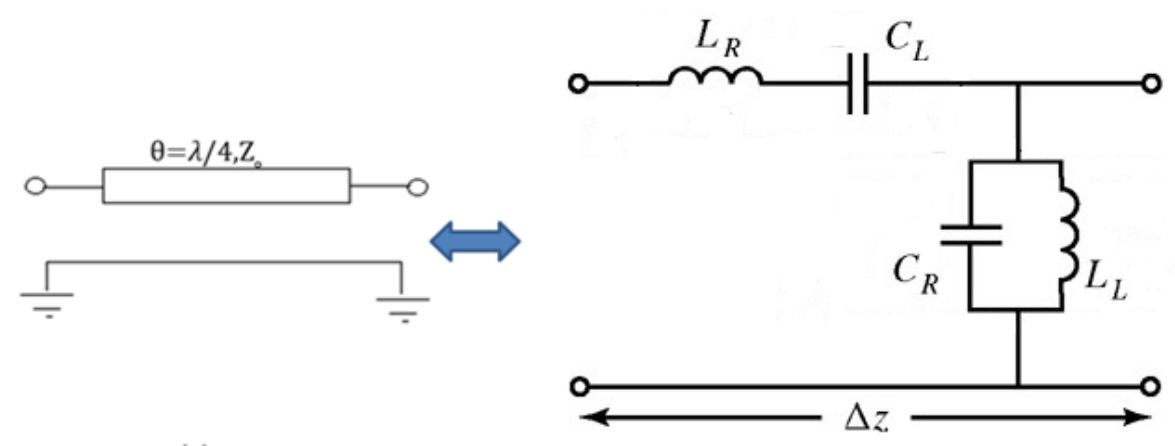

(a)

(b)

Figure 4-6: Schematic representation of (a) Conventional TL (b) CRLHTL

The values of the capacitors and the inductors in the CRLHTL can be determined by equating the ABCD-matrices of the two network circuits of Figure 4-6-a and 4-6-b which are given by [4]:

$\left[\begin{array}{ll}A & B \\ C & D\end{array}\right]_{a}=\left[\begin{array}{cc}\cos \theta & j Z_{o} \sin \theta \\ \frac{j}{z_{o}} \sin \theta & \cos \theta\end{array}\right]$ 
$\left[\begin{array}{ll}A & B \\ C & D\end{array}\right]_{b}=\left[\begin{array}{cc}1-X & Z \\ Y & 1\end{array}\right]=\left[\begin{array}{cc}1-X & j \frac{\left(\omega / \omega_{s e}\right)^{2}-1}{\omega C_{L}} \\ j \frac{\left(\omega / \omega_{s h}\right)^{2}-1}{\omega L_{L}} & 1\end{array}\right]$

where the series and the shunt resonance $\omega_{\text {se }}$ and $\omega_{\text {sh }}$ are $\frac{1}{\sqrt{L_{R} C_{L}}}$ and $\frac{1}{\sqrt{L_{L} C_{R}}}$, respectively.

$X=Z Y=\left(\frac{\omega}{\omega_{R}}\right)^{2}+\left(\frac{\omega_{L}}{\omega}\right)^{2}-K \omega_{L}^{2}$

$\omega_{\mathrm{R}}=\frac{1}{\sqrt{\mathrm{L}_{\mathrm{R}} \mathrm{C}_{\mathrm{R}}}}$

$\omega_{\mathrm{L}}=\frac{1}{\sqrt{\mathrm{L}_{\mathrm{L}} \mathrm{C}_{\mathrm{L}}}}$

$\mathrm{K}=\mathrm{L}_{\mathrm{R}} \mathrm{C}_{\mathrm{L}}+\mathrm{L}_{\mathrm{L}} \mathrm{C}_{\mathrm{R}}$

The balance condition for the circuit network of the TL can be given by:

$\omega_{\text {se }}=\omega_{\text {sh }}$ or $\mathrm{L}_{\mathrm{R}} \mathrm{C}_{\mathrm{L}}=\mathrm{L}_{\mathrm{L}} \mathrm{C}_{\mathrm{R}}$ or $\mathrm{Z}_{\mathrm{L}}=\mathrm{Z}_{\mathrm{R}}$

By assuming a balance case and choosing the characteristic impedance $\mathrm{Z}_{\mathrm{o}}=50 \mathrm{ohm}$ at the center frequency of $5 \mathrm{GHz}$ and with $\theta=90$ degree, the values of the capacitors and inductors can be determined as follows:

$$
\begin{aligned}
& \mathrm{C}_{\mathrm{L}}=\frac{\left(\omega / \omega_{\mathrm{se}}\right)^{2}-1}{\omega \mathrm{Z}_{\mathrm{o}}} \sin \theta \\
& \mathrm{L}_{\mathrm{L}}=\frac{\mathrm{Z}_{\mathrm{o}}\left(\omega / \omega_{\mathrm{sh}}\right)^{2}-1}{\omega} \sin \theta
\end{aligned}
$$

The impedance and the electrical lengths of the longitudinal and transverse branches of the coupler can be obtained from the ABCD matrix in (4-12), thus,

$$
\frac{\sin \theta_{\text {horizontal }}}{\sin \theta_{\text {vertical }}}=\sqrt{2} \frac{Z_{\text {vertical }}}{Z_{\text {horizontal }}}
$$

From (4-15), choosing the wavelength $=\lambda / 4$ for the horizontal and vertical branches, results the angle $90^{\circ}$ and $Z_{\text {vertical }}=\sqrt{2} Z_{\text {horizontal }}$. The characteristic impedance for the branch 56 
line coupler $\mathrm{Z}_{\mathrm{o}}=50 \mathrm{ohms}$, thus, the impedance of vertical branch in the BLC becomes 50 ohms and the impedance of the horizontal is $35.35 \mathrm{ohms}$.

\subsection{Design of a Reduced Size 90 ${ }^{\circ}$ CRLHBLC}

In this section, the design of a compact BLC using lumped element CRLHTL is presented. The compactness is achieved by replacing the RHTL in a conventional BLC with a 90degree CRLHTLs implemented using lumped elements. The feed line also has characteristic impedance of $50 \Omega$. The CRLH branch line coupler is formed using $35.5 \mathrm{ohm}$ and 50 ohm for the horizontal and vertical branches, respectively. The operating frequency bandwidth is chosen to be $300 \mathrm{MHz}(5-5.3 \mathrm{GHz})$. The simulated S-parameters of the horizontal and vertical branches are shown in Figure 4-7 and Figure 4-8.

In the conventional BLC that is shown in Figure 4.3, the branch lines need to be $\lambda / 4$ long since the overall size of the coupler is quite large $(6.604 \times 6.778 \mathrm{~mm})$ over the operating frequency. In addition, the CRLHTL used to implement lumped elements to replace these branch lines. The CRLHTL unit cells, which are used to replace the $50 \mathrm{ohms}$ vertical branch line and $35.35 \mathrm{ohms} \mathrm{horizontal}$ branch line in the conventional branch line coupler of Figure 4.3, are shown in Figures 4-7 and 4-8, respectively [53]. A computer simulation was used to obtain dimensions of the CRLHTL for the required phase shifts and impedance. The $50 \Omega$ vertical line was designed on a ceramic substrate, LTCC technology, with a relative dielectric constant of 7.1, thickness of $127 \mu \mathrm{m}$ and a loss tangent of 0.001 . The de-

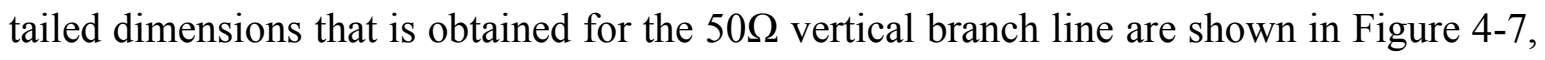
they have a phase shift of 90 degree and an impedance of $50 \Omega$ at $5 \mathrm{GHz}$, with the required for the components of $\mathrm{C}=1.6 \mathrm{pF}$, and $\mathrm{L}=2 \mathrm{nH}$. The simulated $|\mathrm{S} 11|$ and $|\mathrm{S} 21|$ are shown 57 
in Figure 4-6-c and the simulated phase response is in Figure 4-7-b. We can see that the branch line had a bandwidth from 5 to $5.3 \mathrm{GHz},(|S 11|<-20 \mathrm{~dB})$ and a phase shift of 88 degree. Thus, it could be used to design a BLC at $5 \mathrm{GHz}$.

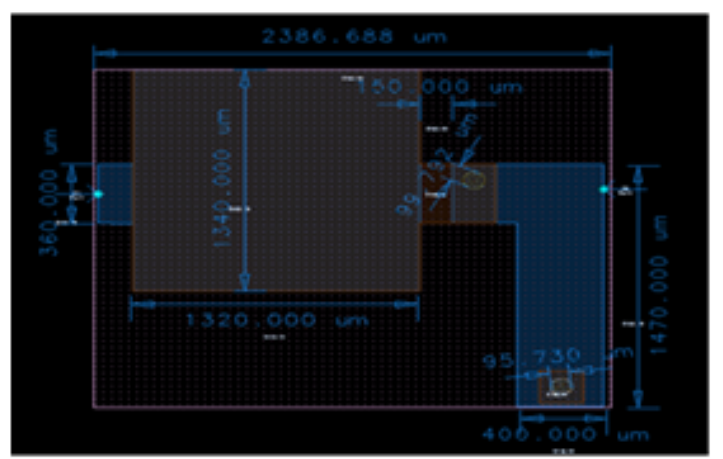

(a)

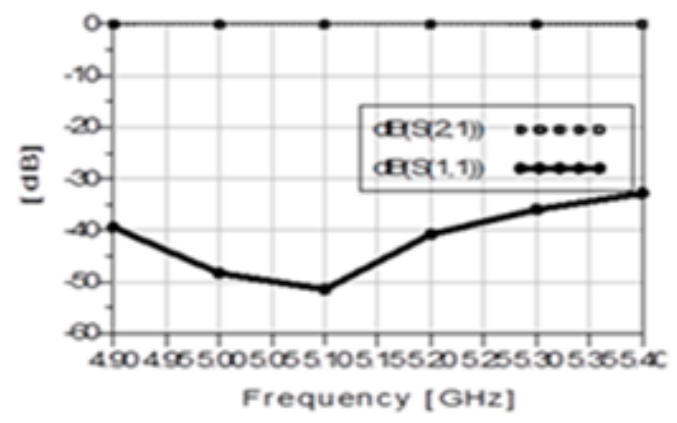

(c)

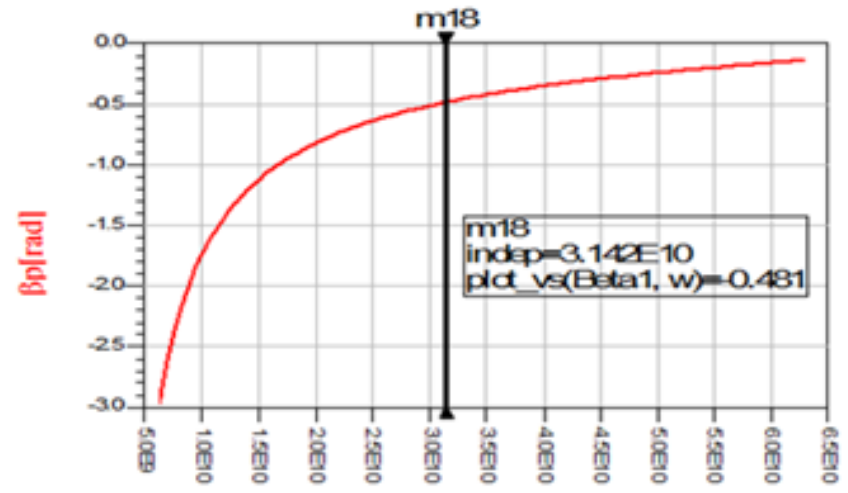

$\omega[\mathrm{rad}](\mathrm{b})$

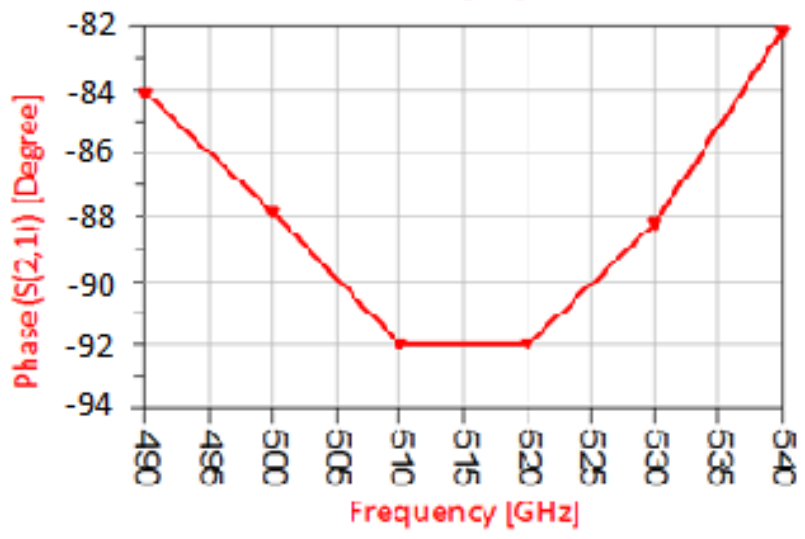

(d)

Figure 4-7: Vertical branch at $50 \Omega$ (a) Layout of the transverse branch, (b) Dispersion diagram and (c) $|S 11|$ and $|S 21|($ d) Phase of $\mathrm{S}(2,1)$

For the $35.35 \Omega$ horizontal line that is shown in Figure 4-8, the width of the microstrip line is needed to be adjusted in order to have an impedance of $50 / \sqrt{2}=35.35 \Omega$. However, this change of dimension would change the operating frequency band of having the 90-degree phase shift required in a BLC. To solve this issue, the values of the capacitors required to 
shift the operating frequency of having 90-degree phase shift back to $5 \mathrm{GHz}$ were obtained using computer simulation. Simulation showed that the required values for the components in the horizontal line were $C=1.4 \mathrm{pF}$ and $\mathrm{L}=1.8 \mathrm{nH}$. The simulated $|S 11|,|S 21|$ and phase response for the 35.35 $\Omega$ horizontal branch line are shown in Figure 4-8. Figure 4-8-c shows that the horizontal branch line had a bandwidth $(|\mathrm{S} 11|<-15 \mathrm{~dB})$ at $5 \mathrm{GHz}$ and Figure 4-8-b shows that the branch line had a phase shift of 85 degree at $5 \mathrm{GHz}$. Thus horizontal branch line satisfied the required characteristic to be used in a BLC.

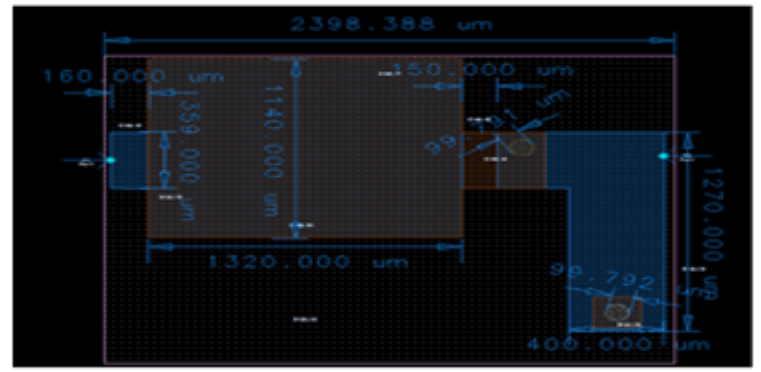

(a)

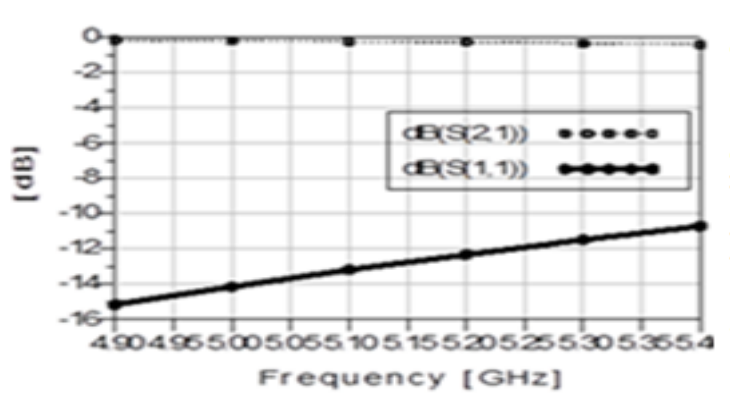

(c)

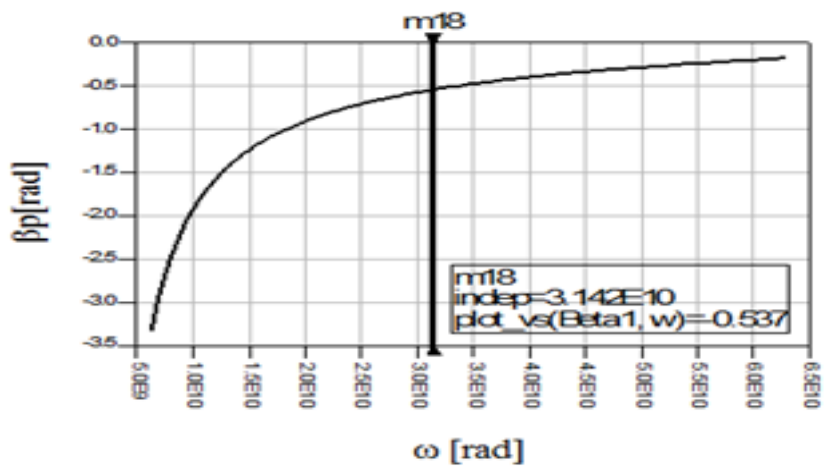

(b)

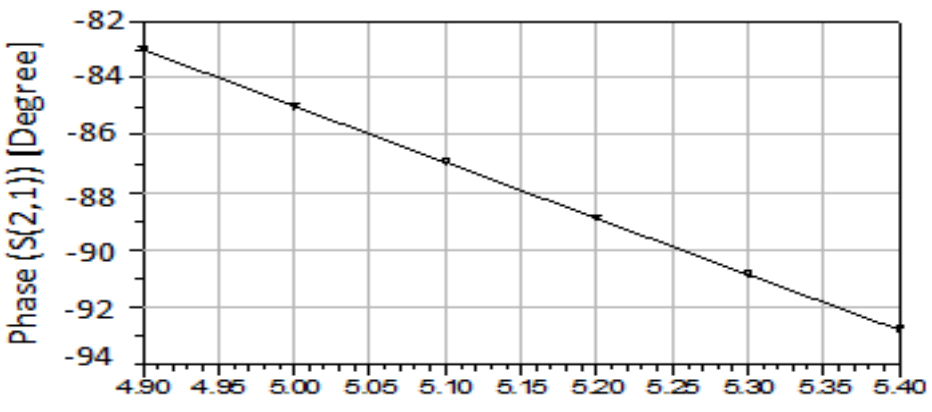

Frequency $[\mathrm{GHz}]$

(d)

Figure 4-8: Horizontal branch at $35 \Omega$ (a) Layout of the transverse branch, (b) Dispersion diagram and (c) $|S 11|$ and $|S 21|$ (d) Phase of $(\mathrm{S}(2,1))$ 
In Figures, 4-7 and 4-8 the unit cell has a phase shift of 88 and 85 degrees respectively. Following that, in Figure 4-9 an extra transmission lines were added to either sides of the unit cells to enable adjusting the phase between the ports. The dimensions of the extra lines are $660 \mu \mathrm{m} \times 201 \mu \mathrm{m}$ at each corner. Thus, these added extra transmission lines have changed the phase shift of the unit cells used to form the BLC, by adding more phase shift to the unit cell, which has different polarity for a regular RHTL and LHTL. The extra phase shift came from the extra transmission lines have taken into accounts to design the new unit cells accordingly to form the coupler shown in Figure 4-9. To get rid of the extra phase shift in the unit cells and to achieve the suitable phase shift for the BLC, the dimensions of the horizontal branch at $35 \Omega$ in Figure 4-8 and the vertical branch at $50 \Omega$ were adjusted to achieve the required phase. From Figures 4-7 and 4-8 the capacitors have 1340 $\mu \mathrm{m} \times 1320 \mu \mathrm{m}$ and $1140 \mu \mathrm{m} \times 1320 \mu \mathrm{m}$, respectively, also the dimensions of the stub inductors in both figures are $1270 \mu \mathrm{mx} 400 \mu \mathrm{m}$. Hence, in Figure 4-9 the dimensions of the capacitors are changed to be for the vertical $1040 \mu \mathrm{mx} 1320 \mu \mathrm{m}$ and for the horizontal 1440 $\mu \mathrm{mx} 1520 \mu \mathrm{m}$, and the stub inductors have the dimensions of $1397 \mu \mathrm{mx} 313 \mu \mathrm{m}$. These changes of dimensions and the effect of the T-junctions of the unit cells used to form the CRLHBLC, was successfully used to return the phase shift of the unit cell to the right phase angle.

In Figure 4-11 the size of the coupler might be reduced much further by reducing the length of the internal transmission lines, but as discussed previously, the internal and the external transmission lines were added to either sides of the unit cell to eliminate the discontinuities effects of the T-junctions at the corners of the coupler. And reducing the length 60 
of the internal TL will require adjustments to the sizes of capacitors and inductors to maintain 90 degrees. To achieve a $22.9 \%$ size reduction in the current design the internal transmission lines have occupied a $625 \mu \mathrm{m}$ in each side of the unit cell. Thus, to save more sizes in CRLHBLC comparing to the conventional coupler, these internal transmission lines have to be reduce as much as can be to achieve the minimum size reduction. On the other hand, reducing the length of the internal transmission lines will occur increasing the size of the capacitors and the stub-inductors of the unit cell, this will leads to overlapping between the inductors inside the coupler shape and will also occur to increase the length of the external line to adjust the T-junction to desired the 90 degrees phase shift.

For instant, adjusting the length of the internal transmission lines to be $382 \mu \mathrm{m}$ instead of $625 \mu \mathrm{m}$ on each side of the unit cell will lead to achieve a coupler size of $5438 \mu \mathrm{mx} 5338$ $\mu \mathrm{m}$, which means a $31.8 \%$ size reduction. Following that, the stub-inductors in each unit cell have reached to the maximum length before start overlapping, as well as the length of the external transmission lines had increased to be $710 \mu \mathrm{m}$ instead of $660 \mu \mathrm{m}$. In conclusion, more simulations are required by changing the length of the internal transmission lines to achieve more size reductions, and to predict how much the phase shift would change then adjust for that by resizing the LC components of the unit cell, for instant the designed CRLHBLC has achieved $22.9 \%$ size reduction comparing to conventional coupler using LTCC technology.

Table 4-1 and 4-2 illustrates the dimensions of the unit cell before and after adding the transmission lines for the 35 and 50 ohms unit cells, respectively. 
Table 4-1: Dimensions of the 35 ohms unit cell before and after adding the TL

\begin{tabular}{|l|l|l|l|}
\hline At 35 Ohm TL & Original cell & $\begin{array}{l}\text { Original cell with a T.Line } \\
\text { element at each end }\end{array}$ & $\begin{array}{l}\text { Adjusted the original cell } \\
\text { and the T. Lines }\end{array}$ \\
\hline $\begin{array}{l}\text { Cell dimension } \\
{[\mu \mathrm{m}]}\end{array}$ & $2238.4 \times 1270$ & $2238.4 \times 1270$ & $2708 \times 1640$ \\
\hline LL $[\mathrm{nH}]$ & 1.55 & 1.55 & 1.94 \\
\hline CL $[\mathrm{pF}]$ & 1.21 & 1.21 & 1.82 \\
\hline LR $[\mathrm{nH}]$ & 0.11 & 0.11 & 0.20 \\
\hline CR $[\mathrm{pF}]$ & 0.59 & 0.59 & 0.75 \\
\hline $\begin{array}{l}\text { Phase shift } \\
{[\mathrm{Degree}]}\end{array}$ & 86.6 & 81.2 & 88.2 \\
\hline S11 $[\mathrm{dB}]$ & -17 & -12 & -23 \\
\hline S21 $[\mathrm{dB}]$ & 0.35 & 0.65 & 0.1 \\
\hline $\begin{array}{l}\text { T.L. LxW } \\
{[\mu \mathrm{m}]}\end{array}$ & NA & $825 \times 200$ & $825 \times 200$ \\
\hline
\end{tabular}

Table 4-2: Dimensions of the 50 ohms unit cell before and after adding the TL

\begin{tabular}{|l|l|l|l|}
\hline At 50 Ohm TL & Original Cell & $\begin{array}{l}\text { Original cell with a T.Line } \\
\text { element at each end }\end{array}$ & $\begin{array}{l}\text { Adjusted the original cell } \\
\text { and the T. Lines }\end{array}$ \\
\hline $\begin{array}{l}\text { Cell dimension } \\
{[\mu \mathrm{m}]}\end{array}$ & $2226.7 \times 1470$ & $2226.7 \times 1470$ & $2533 \times 1540$ \\
\hline $\mathrm{LL}[\mathrm{nH}]$ & 1.52 & 1.52 & 2.20 \\
\hline CL $[\mathrm{pF}]$ & 1.16 & 1.16 & 1.85 \\
\hline LR $[\mathrm{nH}]$ & 0.10 & 0.10 & 0.41 \\
\hline CR $[\mathrm{pF}]$ & 0.31 & 0.31 & 0.55 \\
\hline $\begin{array}{l}\text { Phase shift } \\
{[\mathrm{Degree}]}\end{array}$ & 85.1 & 80.9 & 88.4 \\
\hline S11 $[\mathrm{dB}]$ & -14 & -12 & -25 \\
\hline S21 $[\mathrm{dB}]$ & 0.55 & 1.3 & 0.7 \\
\hline $\begin{array}{l}\text { T.L. LxW } \\
{[\mu \mathrm{m}]}\end{array}$ & NA & $822.5 \times 200$ & $822.5 \times 200$ \\
\hline
\end{tabular}

It well known that adding a transmission line to the circuit should add extra phase shift to that line. In this case, from Tables 4-1 and 4-2, the third column seems to show the opposite where the phase shift had reduced by 5 degrees roughly when adding an extra transmission line to the original unit cell. Generally speaking, there is a difference in phase be- 
tween a right handed line and a left handed line. The phase is opposite for the two, hence when we add a right handed line to a left handed line, we expect the phase to be opposite. In other words if the left handed unit cell increases the phase, then the right handed cell must decrease the phase. To clarify this more, by adding numbers and polarity on the phase shift, a +90 degrees phase shift of a left handed transmission line could be achieved (for example the experimental cell at $3.2 \mathrm{GHz}$ on Page 42), or we could build a right handed cell or line with -90 degrees of phase (for example the experimental cell on Page 42 close to $9 \mathrm{GHz}$ ). In other words, a left handed line has phase lead, and the right handed line has phase lag; therefore if phase lead is labeled as positive then phase lag must be labeled as negative. Thus, if the left handed cell starts with a +90 degrees then adding a right handed line will add phase lag (negative phase). As an example, if the right-handed line is not too long, it happens to subtract 5 degrees from the left-handed cell. Then noting that the total phase is now too low, and then we modified the original cell in the fourth column to get the 5 degrees back again. Then this would be equivalent to having designed the LH unit cell to have +93.2 degrees of phase shift (93.2 degrees of phase lead) then we add -5 degrees (or 5 degrees of phase lag) and we end up with 88.2 degrees. 


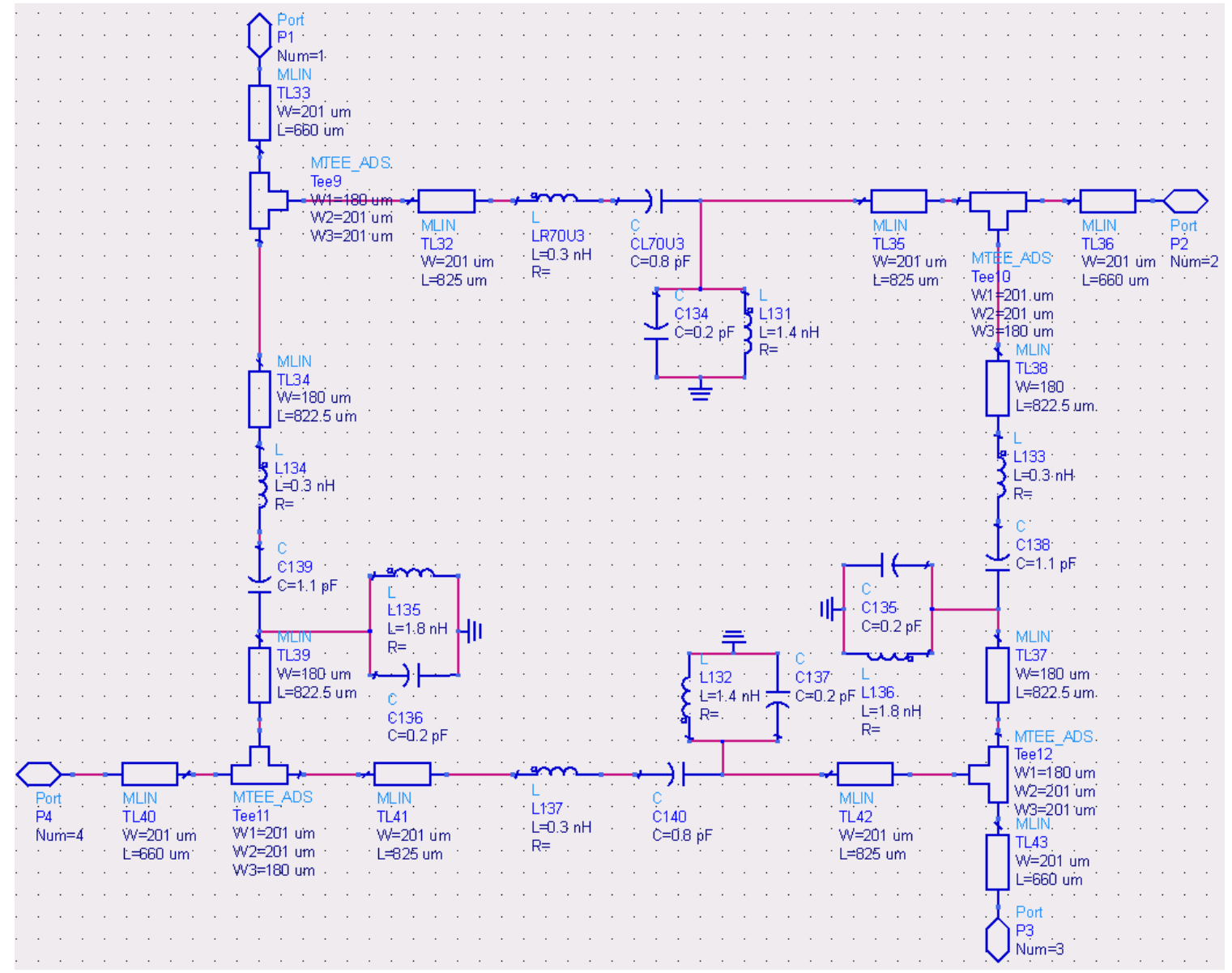

Figure 4-9: Proposed of CRLH branch line coupler

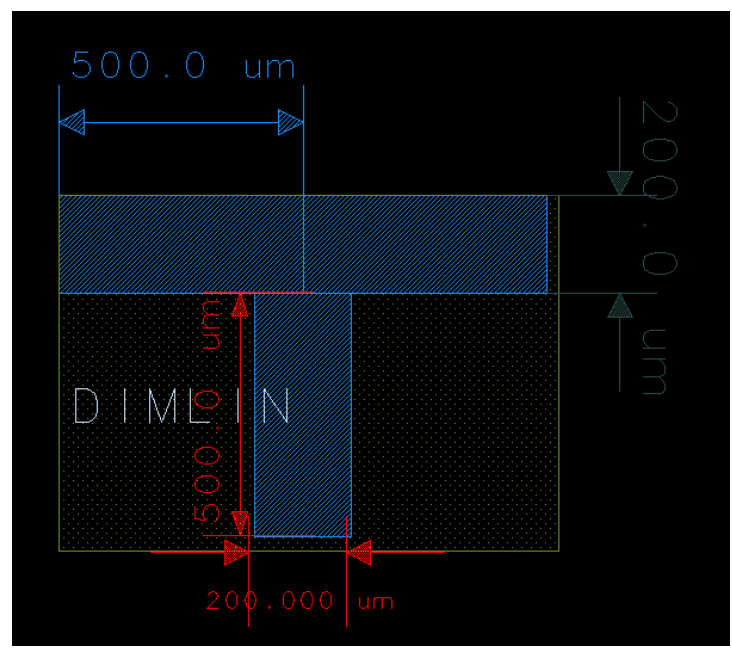

Figure 4-10: Configuration of the T-junction used in CRLHBLC design 64 
With the use of the $35.35 \Omega$ horizontal branch line and $50 \Omega$ vertical branch line that are shown in Figures 4-8 and 4-7, respectively, the CRLHBLC was designed as it is shown in Figure 4-11. The coupler had an area of $5.924 \mathrm{mmx} 5.824 \mathrm{~mm}$ as shown in Figure 4-12. To save space, all stubs are placed inside the square formed by the branches.

Figure 4-11 shows the 3-D view of the CRLHBLC implemented by connecting the layout of the horizontal and the vertical branches with an extra lines on each side connected with a T-junction in each corner to form the coupler. The CRLHBLC is simulated using 3-metal layers on the ceramic substrate LTCC technology with a dielectric constant of 7.1 and a thickness of $127 \mathrm{um}$.

As shown in Figure 4-12 the CRLHBLC which has external transmission lines $875 \mu \mathrm{m}$ length on each corner to enable adjusting the change in phase at each port.

The conventional BLC is bigger than the CRLHBLC because the core of the conventional coupler has longer RHTL than the CRLHTL. The size of the simulated CRLHBLC is $5.924 \mathrm{~mm} \times 5.824 \mathrm{~mm}$ and achieved $22.9 \%$ size reduction in comparison with the conventional coupler. 


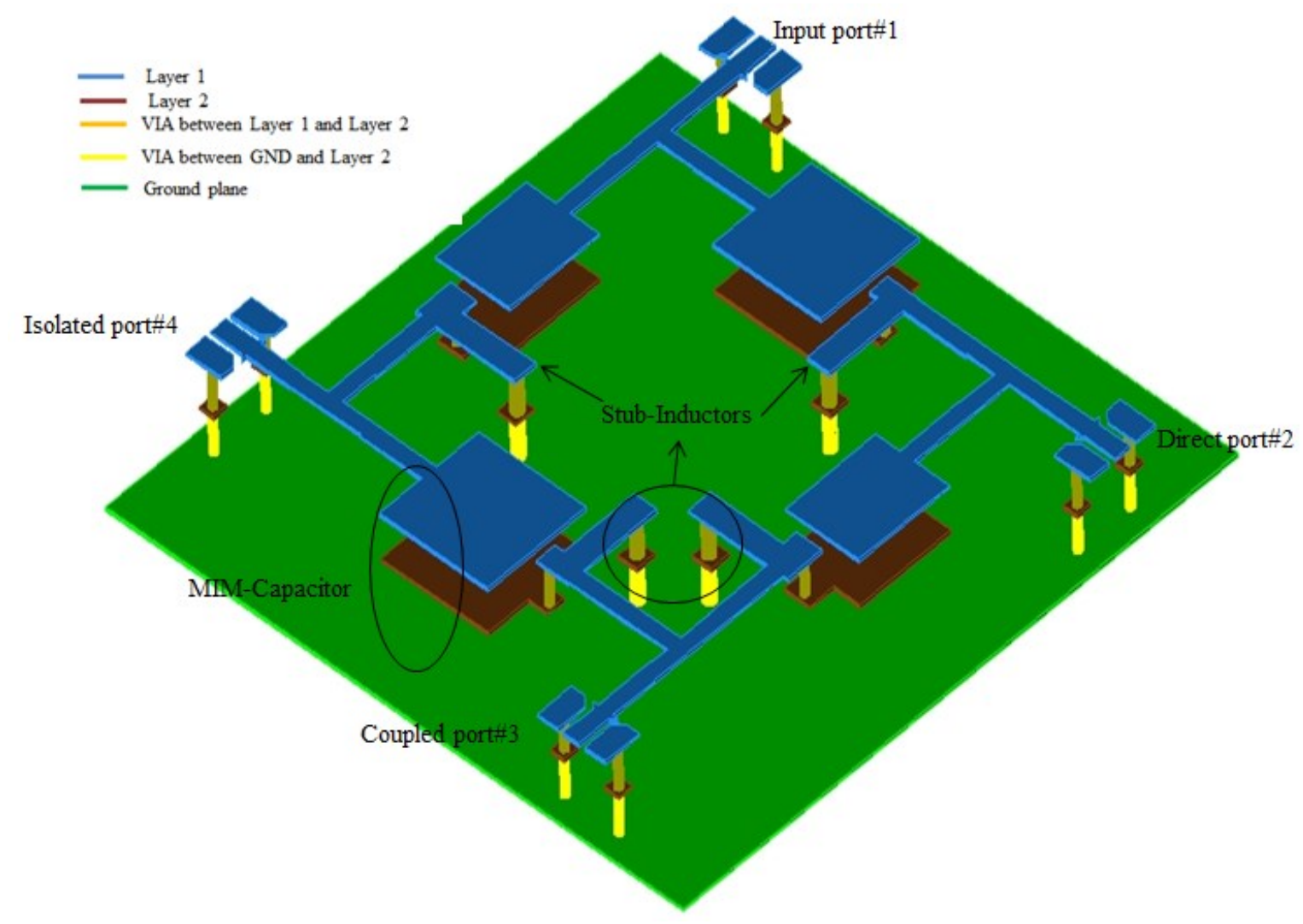

Figure 4-11: 3-D view of the layout of CRLHBLC 


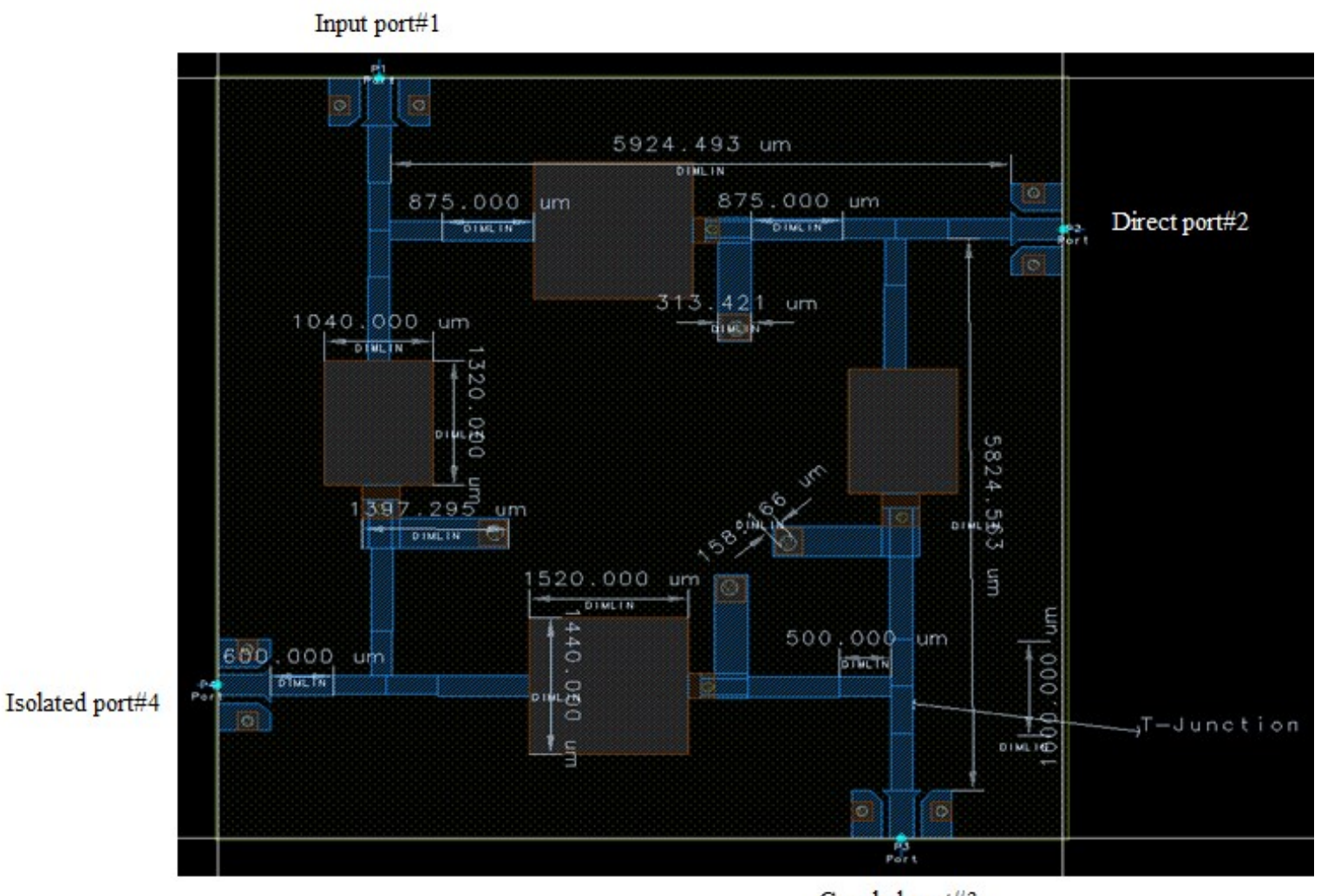

Coupled port\#3

Figure 4-12: Layout of the CRLHBLC 


\subsection{Results and Discussions}

The final simulations of the BLC were carried out in a hybrid simulation mode within Agilent -ADS called a Co-simulation. This allows the placement of the planar models imported from momentum within a single schematic design. When the simulation is run, ADS calls the momentum simulator which analyzes the planar structures within the design using the method-of-moments, and then returns the result to the schematic as a frequencydependent S-parameter file. The ADS schematic simulator then performs a nodal analysis on all of the interconnected elements within the design and produces the final results. Thus, the BLC was analyzed using the Co-simulation technique within ADS.

Figure 4-13 shows the simulation results of the CRLHBLC. The simulated S-parameters of the CRLHTL are shown in Figure 4-13-a, the insertion loss between the input port and the direct port (S21) is close to $-3 \mathrm{~dB}$ over the frequency bandwidth of $(4.5-5.5 \mathrm{GHz})$, the coupling between the input port and the coupled port $(\mathrm{S} 31)$ is close to $-3.5 \mathrm{~dB}$, and the phase difference between the direct port and the coupled port of the coupler is shown in Figure 4-13-d, the phase difference is about 266 - 271 degrees, and Figure 4-13-c shows the return loss for all ports which are less than $-10 \mathrm{~dB}$ over the frequency bandwidth of $(4.5-5.5 \mathrm{GHz})$.

This can be compared to the conventional BLC which was implemented using RLTLs and works at the same frequency band. The proposed CRLHBLC design occupied an area of $77.1 \%$ of the conventional coupler`s size. 

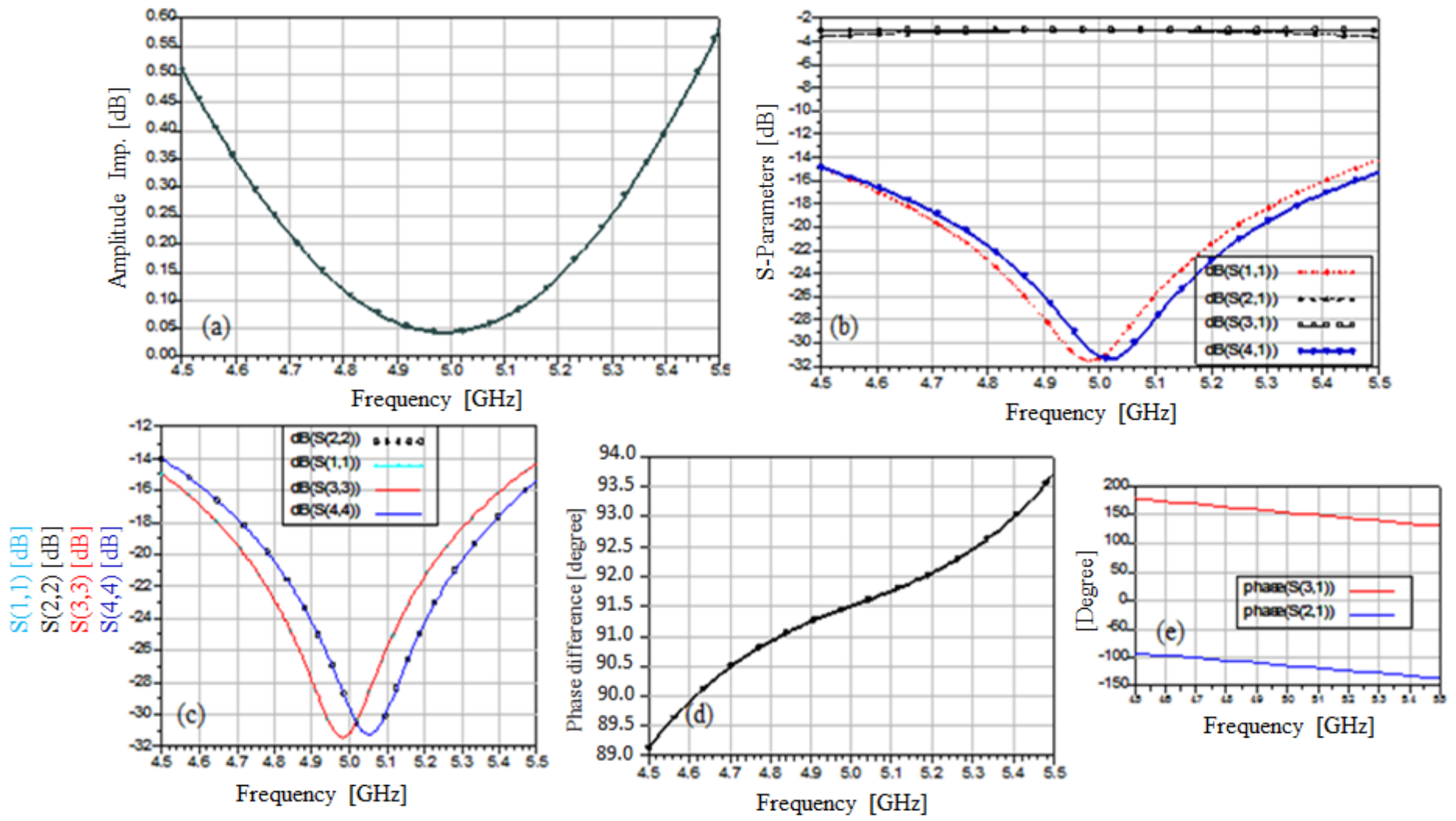

Figure 4-13: Simulation of the CRLHBLC shown in Figure 4-10 (a) Amplitude imbalance,

(b) S-Parameters, (c) Return Loss, (d) Phase difference between port 2 and 1, (e) Individual phase for port 2 and port 3 


\subsection{Chapter Summary}

In this chapter, the design of a compact branch line coupler using CRLHTL is presented. The compactness is achieved by replacing the 90-degree RHTL in a conventional BLC with the CRLHTLs implemented using lumped elements. Computer simulation is used to study the performance of the CRLH-BLC and the results show that the coupler has a bandwidth from 4.5 to $5.5 \mathrm{GHz}$, with $|\mathrm{S} 11|$ and $|\mathrm{S} 41|$ lower than $-10 \mathrm{~dB},|S 21|$ and $|S 31|$ close to $-3.5 \mathrm{~dB}$, and a phase difference closed to 270 degrees between ports 2 and 3 . For comparison, a conventional BLC implemented using the RHTL and working at the same frequency band is designed. Simulation results show that the proposed CRLH-BLC achieved a size reduction of $22.9 \%$ compared with the conventional RHTL BLC. 


\section{Chapter 5}

\section{Conclusions and Future Work}

\subsection{Conclusion}

This thesis has investigated the development of metamaterial transmission lines and their successful implementation in the development of a CRLH reduced-size branch line coupler.

The proposed CRLHTL unit cell is implemented using lumped elements (MIM-capacitors and shorted stub-inductors) to replace the conventional RHTL. The CRLHTL is tested over the bandwidth of 4.5-5.5 GHz. The dispersion diagram of the transmission line shows a negative propagation phase constant and a positive propagation phase constant above the center frequency of $5 \mathrm{GHz}$. The fabricated transmission line has zero-degree phase shift at the resonance frequency of $5 \mathrm{GHz}$. Thus, this transmission line behaves like a short circuit at resonance frequency. Also, the dispersion diagram of the fabricated TL and the simulated TL shows a 90 degree shift at $3.2 \mathrm{GHz}$. This transmission line was initially mistakenly designed for a resonant frequency of $5 \mathrm{GHz}$ rather than the 90 degree frequency at $5 \mathrm{GHz}$, hence the 90 degree frequency ended up at $3.2 \mathrm{GHz}$. Thus, it can be used to design many components at $3.2 \mathrm{GHz}$, such as antennas, notch filter and diplexer.

For application use, a new CRLHTL is designed which provides flexibility to achieve the desired impedance that facilitates the design of passive components in microwave frontends, in order to achieve good performance. This transmission line is used to build a branch 
line coupler using the LTCC technology. For comparison, a conventional BLC implemented using Right Handed Transmission Lines (RHTL) has also been designed and studied. Simulation results have shown that our proposed design achieved an area reduction of $22.9 \%$ compared with a conventional RHTL BLC, over a bandwidth of about $300 \mathrm{MHz}$ (5$5.3 \mathrm{GHz}$ ). Table 5.1 gives a comparison between the CRLH coupler and the conventional coupler.

Table 5-1: Comparisons of the CRLHBLC with the conventional RHBLC

\begin{tabular}{|l|l|l|}
\hline & Conventional BLC & CRLH BLC (this work) \\
\hline Simulation on ceramic substrate- & 2-metal layers & 3-metal layers \\
\hline Frequency range [GHz] & $4.5-5.5$ & \\
\hline Fractional BW [dB] & 20 & $4.5-5.5$ \\
\hline Dimension $(\mathrm{mm})^{2}$ & $6.604 \times 6.778$ & 20 \\
\hline Relative area & $100 \%$ & $5.924 \times 5.824$ \\
\hline Power Dividing Imbalance $[\mathrm{dB}]$ & 0.1 & $77 \%$ \\
\hline Max $\left(\mathrm{S}_{11}\right)$ over bandwidth $[\mathrm{dB}]$ & -12 & 0.4 \\
\hline IL at $\mathrm{f}_{\mathrm{o}}[\mathrm{dB}]$ & 3.1 & -12 \\
\hline Isolation $[\mathrm{dB}]$ less than & 10 & 3.3 \\
\hline Transmission Medium & Microstrip & CRLHTL \\
\hline
\end{tabular}




\subsection{Future Work}

Future research could be carried out to develop accurate passive components using LTCC technology at frequencies in the C-band. Also, utilizing the composite right left handed transmission line of this work, the design of the $5 \mathrm{GHz}$ band branch line coupler in ceramic substrate LTCC technology can be undertaken to increase the performance and reduction in size. 


\section{Appendices}

\section{Appendix A Test Plan for LTCC Structures}

The LTCC wafer consists of 3 layers of DuPont 9K7 with gold metallization. The structures to be tested are located over the surface of the wafer in various locations, as shown in Figure (A-1). The wafer is labeled LCCUCHE for identification.

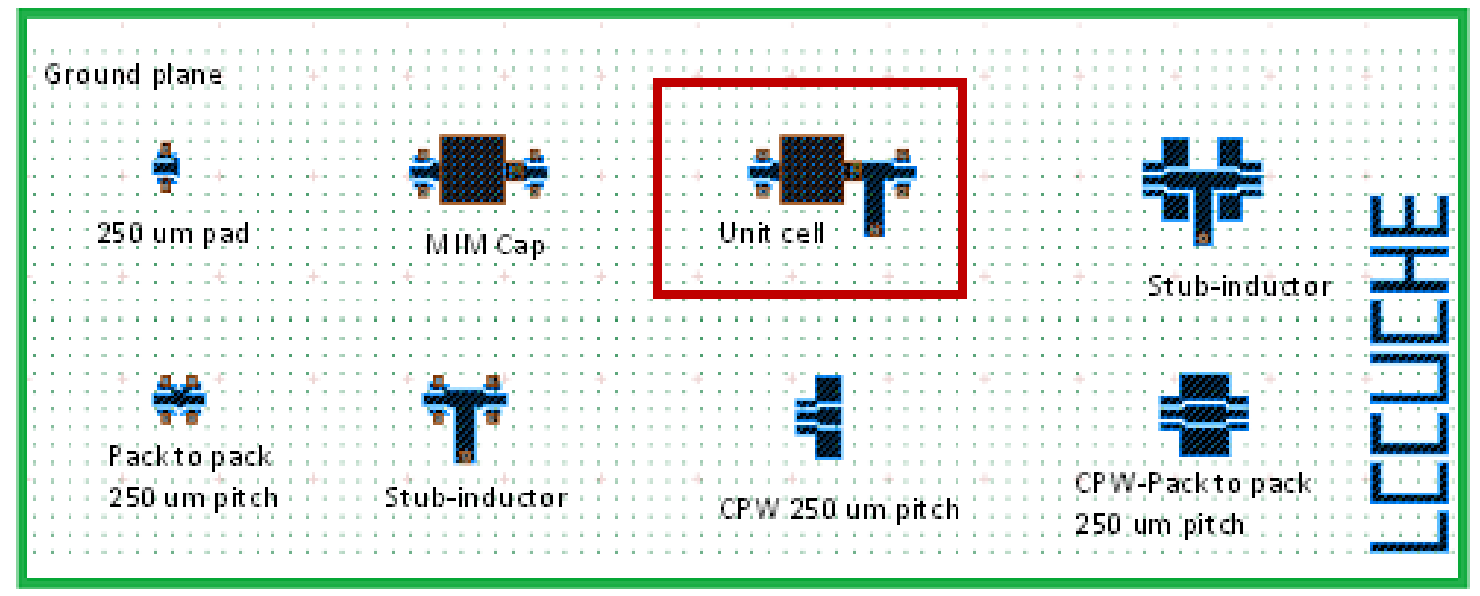

Figure A-1: LTCC Wafer LCCUCHE

The various devices consist of capacitors, inductive microstrip line stubs, microstrip lines, coplanar microstrip lines, grounded coplanar microstrip lines, and artificial transmission lines. Test structures for probe and landing pad de-embedding are also included.

Equipment

The following equipment will be needed:

250 um pitch, $50 \Omega$ ground-signal-ground probes

Probing station, and appropriate accessories (cables, calibration kits, etc.)

2 port vector network analyzer 


\section{Testing}

Testing will consist of measuring the S-parameters of each device on the wafer. Most are 2 port devices, although there are some 1 port structures.

Frequency Range

The measurements will be made in 1 frequency ranges. All structures located in the wafer, will be measured from $1 \mathrm{GHz}$ to $20 \mathrm{GHz}$.

\section{Calibration and De-embedding}

Calibration of the probes will be required. This may be done with the appropriate calibration kit. De-embedding of the pads will be done offline, mathematically after measurements, thus all measurements will include the landing pads.

\section{Probe Landing}

The appropriate landing target for the probes is shown in A-2.

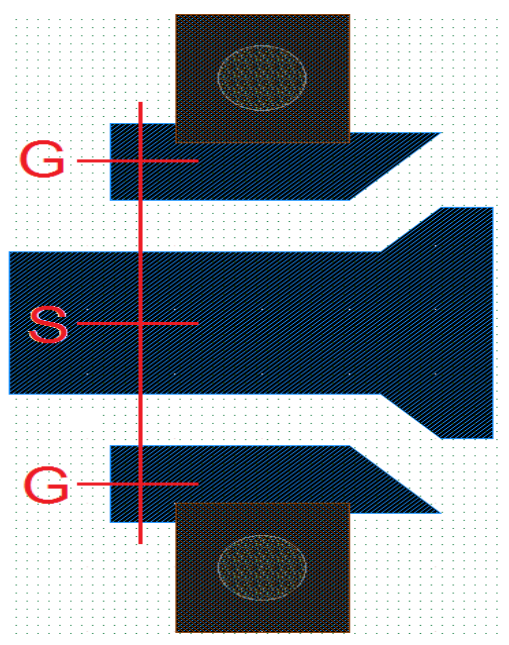

Figure A-2: Probe Landing Target 


\section{Block Diagram of the Test Setup}

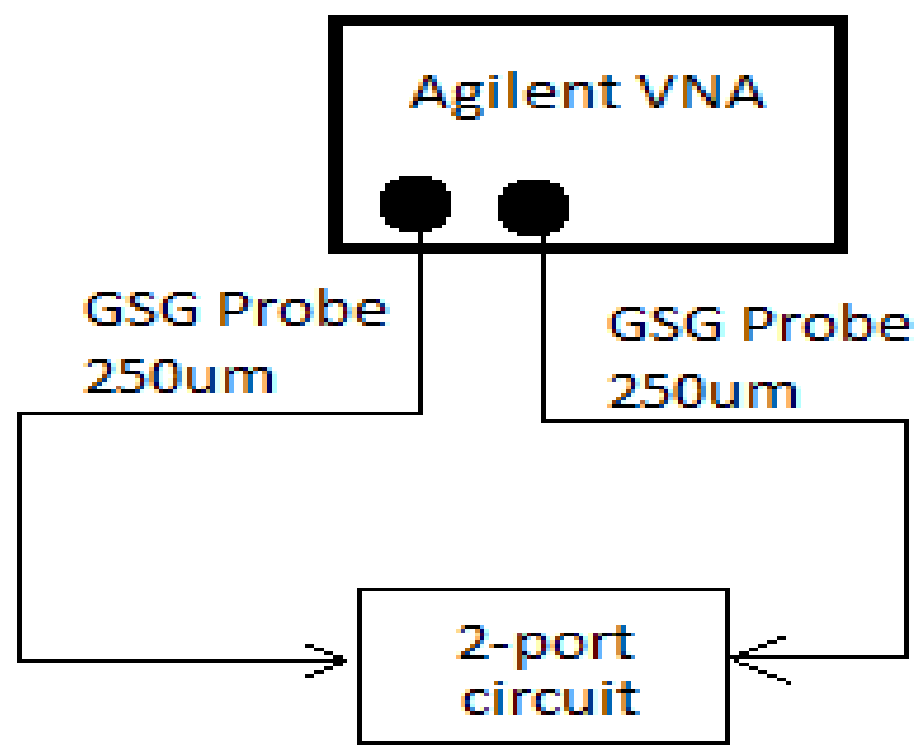

Figure A-3: Test setup for measuring the 2-port transmission line 
Appendix B Calculating the effective dielectric constant

$$
\begin{aligned}
& \text { If } \frac{w}{h}<1 \\
& \varepsilon_{\text {eff }}=\frac{\varepsilon_{r}+1}{2}+\left(\frac{\varepsilon_{r}-1}{2}\right)\left[\frac{1}{\sqrt{\left(1+\frac{12 h}{e}\right)}}\right]+0.04\left(1-\frac{w}{h}\right)^{2} \\
& \text { If } \frac{w}{h}>=1 \\
& \varepsilon_{\text {eff }}=\frac{\varepsilon_{r}+1}{2}+\left(\frac{\varepsilon_{r}-1}{2}\right)\left[\frac{1}{\sqrt{\left(1+\frac{12 h}{e}\right)}}\right]
\end{aligned}
$$

where,

$\varepsilon_{e f f}=$ Effective dielectric constant

$\varepsilon_{r}=$ dielectric constant of the material

$w=$ width of the trace

$h=$ separation of the trace from ground plane

$e=$ trace thickness 


\section{Appendix C Overview of Shifting Reference Planes}

To obtain the S-parameters of the CRLHTL unit cell, the reference plane shifting method is applied [24]. Figure C-1 shows that the same length of microstrip lines are added to each side of the CRLHTL, where $\left(\mathrm{L} 1=\mathrm{L}_{2}=\mathrm{l}\right)$. The reference planes for the unit cells are, at $\mathrm{Z}_{1}$ $=0$ and $\mathrm{Z}_{2}=0$. Using $\mathrm{ADS}$ momentum simulation, the network parameters were obtained at reference planes at $Z_{1}=L_{1}$ and $Z_{2}=L_{2}$. The shift of the reference planes from $Z_{1}=L_{1}$ and $Z_{2}=L_{2}$ to $Z_{1}=0$ and $Z_{2}=0$ have been applied to obtain the network parameters for the CRLHTL [3][4][21].

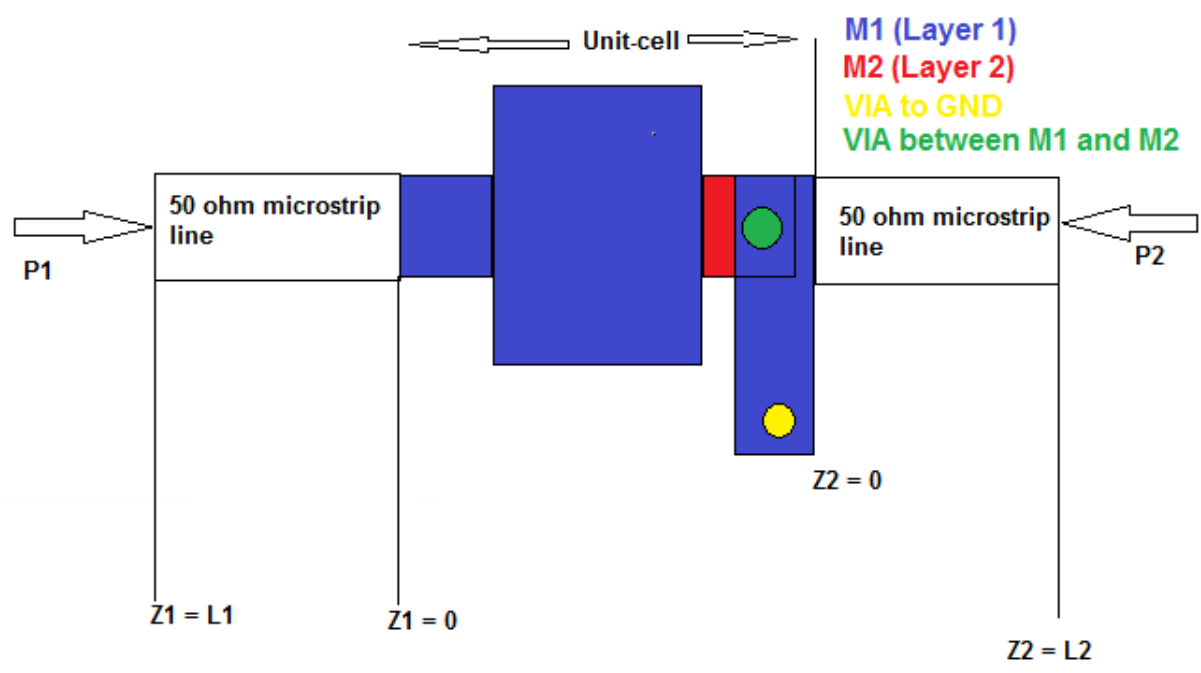

5-1: Reference plane shift for the metamaterial transmission line

A momentum simulation was used to obtain the S-parameters for the full structure shown in Figure 3-9, where the reference plane $Z_{1}=L_{1}$ and $Z_{2}=L_{2}$. The ABCD matrices of the full structure, the microstrip line, and the CRLHTL, respectively, are:

$\left[\begin{array}{ll}A_{1} & B_{1} \\ C_{1} & D_{1}\end{array}\right],\left[\begin{array}{ll}A_{2} & B_{2} \\ C_{2} & D_{2}\end{array}\right]$, and $\left[\begin{array}{ll}A & B \\ C & D\end{array}\right]$ 
$\left.\left[\begin{array}{ll}A_{1} & B_{1} \\ \mathbf{C}_{1} & \mathbf{D}_{1}\end{array}\right]=\left[\begin{array}{ll}A_{2} & \mathbf{B}_{2} \\ \mathbf{C}_{2} & \mathbf{D}_{2}\end{array}\right] \mid \begin{array}{ll}\mathbf{A} & \mathbf{B} \\ \mathbf{C} & \mathbf{D}\end{array}\right]\left[\begin{array}{ll}\mathbf{A}_{2} & \mathbf{B}_{2} \\ \mathbf{C}_{2} & \mathbf{D}_{2}\end{array}\right]$

Where $\left[\begin{array}{ll}A_{1} & B_{1} \\ C_{1} & D_{1}\end{array}\right]$ is the $A B C D$ matrix of the whole structure, and

$\left[\begin{array}{ll}A_{2} & B_{2} \\ C_{2} & D_{2}\end{array}\right]$ is the $A B C D$ matrix of the microstrip lines, and $\left[\begin{array}{ll}A & B \\ C & D\end{array}\right]$ is the $A B C D$ matrix of the unit cell.

from matrix theory:

$\left[\begin{array}{ll}A & B \\ C & D\end{array}\right]=\left[\begin{array}{ll}A_{2} & B_{2} \\ C_{2} & D_{2}\end{array}\right]^{-1}\left[\begin{array}{ll}A_{1} & B_{1} \\ C_{1} & D_{1}\end{array}\right]\left[\begin{array}{ll}A_{2} & B_{2} \\ C_{2} & D_{2}\end{array}\right]^{-1}$

The S-parameters of the unit cell can be extracted from the ABCD matrix. As for the capacitor and the shunt inductor, similar methods can be applied and the S parameters can be obtained and the circuit parameters can then be extracted [23]. 


\section{References}

[1] M. Yagoub, Introduction to microwave devices, class notes for ELG 7100 course, Ottawa University, 2011.

[2] LTCC Design Guideline, CMC Microsystems, Canada, 2011.

[3] C. Caloz and T. Itoh, Electromagnatic Metamaterials (Transmission Line theory and microwave application), Wiley IEEE Press $1^{\text {st }}$ edition, 2005.

[4] D. Pozar, Microwave Engineering, $3^{\text {rd }}$ edition, John Wiley \& Sons, Inc, 2005.

[5] B. Zhao, Composite Right Left-Handed (CRLH) microstrip resonant antennas, Master thesis, University of Cincinnati U.S. state of Ohio, 2005.

[6] D. Pozar, Microwave.Engineering, $2^{\text {nd }}$ edition, John Wiley \& Sons, Inc, 2003.

[7] A. Lai, Left-handed metamaterials for microwave Engineering applications, Doctoral thesis, Department of Electrical Engineering University of California, Los Angeles, 2009.

[8] C. Caloz, C. Chang, and T. Itoh, "Full-wave verification of the fundamental properties of left-handed materials in waveguide configurations," Journal of Applied Physics, pp. 1-4, vol. 90, 2001.

[9] Xin Hu, Some studies on metamaterial transmission lines and their applications, Doctoral thesis in Electromagnetic Theory, Royal Institue of Technology, Stockholm, Sweden 2009.

[10] C. Li, Y. Liu, and F. Li, "Analysis of composite right left-handed coplanar waveguide zeroth-order resonators with application to a band-pass filter," Piers Online, vol. 3, pp. 599-602, 2007.

[11] A. Rennings, C. Caloz, and P. Waldow, "Metal insulator metal CRLH series mode zeroth order resonant antenna implemented in LTCC technology," Asia-Pacific Microwave Conference, pp. 1-4, 2007.

[12] S. Mao and Y. Chueh,"Broadband composite right left-handed coplanar waveguide power splitters with arbitrary phase responses and balun and antenna applications," IEEE Transactions on Antennas and Propagation, pp. 243-250, vol. 54, 2006.

[13] C. Caloz, "Novel composite right left-handed coupled line directional coupler with arbitrary coupling level and broad bandwidth," IEEE Microwave Theory and Techniques, vol. 52, pp. 980-992, 2004. 
[14] D. Upadhyay, "Design of novel improved unit cell for composite right left handed transmission line based microwave circuits," International Journal of Engineering Science and Technology, vol.3, pp. 4962-4967, 2011.

[15] R. Stephane and R. Marcelli "Composite right left handed based devices for microwave applications," National Institute for Research and Development in Microtechnologies, Bucharest, Romania, pp. 91-112, 2006.

[16] W. Liu, "An unequal power divider using composite right left handed transmission line coupler," IEEE MTT-S International Microwave Symposium Digest, pp. 216-219, 2008.

[17] I. Hsiang and C. Caloz "Arbitrary dual-band branch line coupler using CRLH transmission lines," IEEE Microwave Theory and Techniques, vol. 52, pp. 11421149, 2004.

[18] A. Levy and L. Habib, "Optimisation of a microstrip left handed transmission line using circuit modelling," IEEE Transactions on Antennas and Propagation, pp. 2133-2143, 2010.

[19] R. Keshavarz, "Compact and broadband backward coupler with high coupling level based on composite right left-handed transmission lines," Iranian Conference on Electrical Engineering, pp.2195-2199, 2011.

[20] R. Amaya, Waves and impedances on transmission lines, Class notes, Carleton University, 2010.

[21] K. Chang, Handbook of microwave and optical components, vol. 1, John Wiley, New York, 1989.

[22] H. Nguyen and C. Caloz, "Dual-band CRLH branch line coupler in MIM technology," Microwave and Optical Technology Letters, vol. 48, pp. 2331-2333, 2006.

[23] S. Hall, G. Hall, and J. McCall, High speed digital system design, 1st edition, Wiley IEEE Press, 2000.

[24] K. Whites, Properties of S-matrices shifting reference plane, Class note, Lecture16, http://whites.sdsmt.edu/classes/ee481/notes/481Lecture16.pdf, 2012.

[25] S. Gedney, Periodic structures and floquet's theorem, class note, University of Kentucky, pp. 3-37, 2011.

[26] G. Eleftheriades, "EM transmission line metamaterials," Elsevier Ltd, vol.12, pp. 30-41, 2009. 
[27] I. Haroun, Study and implementation of lower ground CPW transmission line for the design of V-band Si-based transcever blocks, Ph.D Thesis, Carleton University, 2010.

[28] I. Haroun, Y. Hsu and D. Chang, "60-GHz rat-race coupler using LG-CPW transmission lines in IPD technology," IEEE Asia Pacific Microwave Photonics, pp. 284-287, 2011.

[29] S. Hong and M. Lancaster, Microstrip filters for RF microwave applications, $1^{\text {st }}$ Edition, Wiley Interscience, 2001.

[30] L. Chiu and Q. Xue, "Wideband parallel strip $90^{\circ}$ hybrid coupler with swap," Institution of Engineering and Technology, vol. 44, pp. 687-688, 2008.

[31] Y. Chun, "Compact wide band branch line hybrid," IEEE Microwave Theory andTechniques, vol. 54, pp. 704-709, 2006.

[32] A. Bader, M. Alqahtani, and M. Alkanhal, "New compact wide band branch line couplers," European Microwave Conference (EuMC), pp. 1159-1162, 2009.

[33] H. Mazhar, "Optimization and compact a wide band 3dB multisection quadrature coupler for $900 \mathrm{MHz}, "$ International Conference Mobile IT Convergence (ICMIC), vol. 9, pp. 43-48, 2011.

[34] R. Levy, "Design considerations for lumped element microwave filters", IEEE Microwave Theory and Techniques, pp. 183-192, vol. 31, 1988.

[35] R. Levy, Directional couplers in advances in microwaves, Academic Press, pp. 184-191, vol. 1, 1966.

[36] Agilent Technology, Overview on interdigital capacitor design, Agilent EEsof EDA, www.agilent.com/find/eesof, 2011.

[37] E. Loskot, S. Leppavuori and A. Kourbanov "Miniaturized branch line directional coupler on low temperature cofired ceramic board," European Microwave Conference, pp. 1-4, 2001.

[38] H. Zeng and G. Wang, "Miniaturization of branch line coupler using CRLH-TL," Radioengineering, vol. 21, pp.606-610, 2012.

[39] H. Xu, G. Wang, and J. Liang, "Novel CRLH TL metamaterial and compact microstrip branch line coupler application," Electromagnetics Research, vol. 20, pp.173-186, 2011. 
[40] Hs. Lu, Y. Kuo, P. Huang and Y. Long, "Dual-band CRLH branch-line coupler in LTCC by lump elements with parasite control," IEEE MTT-S International Microwave Symposium Digest, pp. 393-396, 2010.

[41] G. Eleftheriades and R. Islam, "Enabling RF microwave devices and antennas using negative refractive index transmission lines metamaterials," Loughborough Antennas and Propagation Conference, pp. 13-18, 2007.

[42] E. Hecht, Optics, 4th edition, Addison Wesley, 2001.

[43] M. Grzegorczyk, "Cerenkov radiation in materials with negative permittivity and permeability," Optics Express, vol. 11, pp. 723-734, 2003.

[44] V. Veselago, L. Braginsky, V. Shklover, and C. Hafner, "Negative refractive index materials," Journal of Computational and Theoretical Nanoscience, vol.3, pp. 1$30,2006$.

[45] W. Padilla, D. Basov and D. Smith, "Negative refractive index metamaterial," Elsevier Ltd, vol. 9, pp. 7-8, 2006.

[46] S. Smith, The scientist and engineer 's guide to digital signal processing, California Technical, 1998 .

[47] D. Finkenthal and B. Greco, "Introduction to electromagnatic spectrum," General Atomic, pp.1-27, 1996.

[48] R. Flickenger, Wireless networking in the developing world, $1^{\text {st }}$. edition, Lulu.com, 2006.

[49] R. Marques, F. Mesa, J. Martel and F. Medina, "Comparative analysis of edge and broadside couple split ring resonators for metamaterial design," IEEE Antennas and Propagation Society, vol. 51, pp. 2572-2581, 2003.

[50] R. Shelby, D. Smith and S. Schultz, "Experimental verification of a negative index of refraction," Science, vol. 292, pp.77-79, 2001.

[51] D. Smith and N. Kroll, "Negative refractive index in left-handed materials," The American Physical Society, vol. 85, pp. 2933-2936, 2000. 
[52] C. Caloz and T. Itoh, "Novel microwave devices and structures based on the transmission line approach of metamaterials," IEEE MTT-S International Microwave Symposium Digest, vol. 1, pp. 195-198, 2003.

[53] G. Eleftheriades, A. Iyer and P. Kramer, "Planar negative refractive index media using periodically L-C loaded transmission lines," IEEE Transactions on Microwave Theory and Techniques, vol. 50, pp. 2702-2712, 2002.

[54] N. Engheta and R. Ziolkowski, Metamaterials physics and engineering explorations, (eBook), 2006.

[55] M. Mukherjee, Advanced Microwave and Millimeter Wave Technologies Semiconductor Devices Circuits and Systems, InTech, 2010. 